\title{
Glow Discharge Mass Spectrometric Determination of Selected Biological Species
}

Xiaqing $\mathrm{Xu}$

Follow this and additional works at: https://researchrepository.wvu.edu/etd

\section{Recommended Citation}

Xu, Xiaqing, "Glow Discharge Mass Spectrometric Determination of Selected Biological Species" (2017). Graduate Theses, Dissertations, and Problem Reports. 6989.

https://researchrepository.wvu.edu/etd/6989

This Dissertation is protected by copyright and/or related rights. It has been brought to you by the The Research Repository @ WVU with permission from the rights-holder(s). You are free to use this Dissertation in any way that is permitted by the copyright and related rights legislation that applies to your use. For other uses you must obtain permission from the rights-holder(s) directly, unless additional rights are indicated by a Creative Commons license in the record and/ or on the work itself. This Dissertation has been accepted for inclusion in WVU Graduate Theses, Dissertations, and Problem Reports collection by an authorized administrator of The Research Repository @ WVU.

For more information, please contact researchrepository@mail.wvu.edu. 


\title{
Glow Discharge Mass Spectrometric Determination of Selected Biological Species
}

\section{Xiaqing Xu}

Dissertation submitted to the Eberly College of Arts and Sciences at West Virginia University in partial fulfillment of the requirements for the degree of

\author{
Doctor of Philosophy \\ in \\ Chemistry
}

\author{
Fred L. King, Ph.D., Chair \\ Glen P. Jackson, Ph.D. \\ Stephen J. Valentine, Ph.D. \\ Justin A. Legleiter, Ph.D. \\ Patrick S. Callery, Ph.D. \\ C. Eugene Bennett Department of Chemistry \\ Morgantown, West Virginia \\ 2017
}

Keywords: Pulsed glow discharge, Mass spectrometry, Solution residue analysis, Cysteine, Copper peptide, lodine species

Copyright 2017 Xiaqing Xu 


\title{
Abstract \\ Glow Discharge Mass Spectrometric Determination of Selected Biological Species
}

\author{
Xiaqing Xu
}

Glow discharge mass spectrometry (GDMS) is widely used for trace elemental analysis of solid samples. A glow discharge source employs cathodic sputtering to release cathode species to the gas phase and subsequent collisional ionization of the sputtered species in the plasma. lons representing the cathode material are differentiated by mass-to-charge values and then recorded by mass spectrometry. A pulsed radio frequency (RF) glow discharge time-of-flight mass spectrometry (GD-TOF MS) is employed in this study for its unique advantages, including the capability of direct analysis of nonconducting solids, temporal separation of ionization mechanisms, and the ability to acquire a complete spectrum within a single pulse cycle.

Although GD-TOF MS exhibits superior performance in direct solid analysis, its application to solution samples faces several problems arising from the solvent. In this study, analyte solutions are converted to dry residues on a conducting cathode surface involving the deposition of microliter volumes of analyte solution and subsequent drying in vacuum desiccator before conventional GDMS analysis. By this means, analytes 
including cysteine, glycyl-L-histidyl-L-lysine copper (GHK-Cu) complex and iodinated organic compounds are successfully sampled by GD plasma for mass spectrometric measurement.

Cysteine analysis is a follow-up study based on previous findings in this lab. Cysteine residues analyzed by the current GDMS system reveals a characteristic ion at $m / z 76$ in the afterpeak regime. However, the cysteine residue prepared previously in a $\mathrm{Cu}$ reservoir only produces a very weak characteristic ion signal and this ion signal dissipates quickly over time, indicating significant sample loss in the sputtering process. So, a new sample preparation method was introduced, involving adding a layer of paper material on top of the cathode surface. The layer is designed to provide a better support to the solution residue. In particular, hydrophilic membrane filter as the layer material allows even distribution of the cysteine solution added, and is expected to trap the residue inside the pores as well as on the membrane surface. Residue prepared on the membrane shows superior performance in that an intense and long-lasting cysteine fragment ion signal can be obtained. However, the reproducibility of this method needs improvements, as can be seen from the quantitative results. Comparison of GD results between cysteine and deuterated cysteine fails to prove that the cysteine fragment at $\mathrm{m} / \mathrm{z} 76$ is produced from loss of carboxylic acid group.

GDMS analysis of GHK-Cu complex was completed with dry residues formed on a silver cathode. Residue analysis in a pulsed plasma only produces a distinct $\mathrm{Cu}^{+}$ion signal regarding the analyte in the afterpeak regime. No information regarding the peptide structure was detected. However, the stoichiometric ratio of $\mathrm{Cu}$ element to the 
peptide is known (1:1), enabling the quantification of copper peptide to be completed with $\mathrm{Cu}$ measurement. Before quantification, factors including power, pressure, pulse width, duty cycle and sampling distance were evaluated for their influences on the $\mathrm{Cu}^{+}$ ion signal and optimal conditions were located for the best sensitivity. Subsequently, quantification was completed with GHK-Cu residues prepared from five concentrations and the calibration curve obtained shows good linearity.

lodinated organic compounds, specifically three thyroid hormones (THs) were analyzed by GDMS as well. Because iodine has a high ionization potential (10.45 eV), a helium plasma was established within the GDMS system and its efficiency in sputtering and ionization was proved in a comparison with argon plasma for the analysis of blank $\mathrm{Cu}$ and triiodothyronine (T3). Analysis of THs residues in a pulsed helium plasma reveals a similar pattern and only an $\mathrm{I}^{+}$ion signal found in the afterpeak is of sufficient intensity for further analysis. Quantification of diiodothyronine was demonstrated as an example and the calibration curve shows good linearity. Cross comparison among the three THs reveals that iodine atoms found at different positions within organic molecules are ionized to the same extent in GDMS. 


\section{DEDICATED TO}

\section{My parents and family}




\section{Acknowledgement}

I am very grateful to my advisor Dr. Fred King for his continuous support and encouragement along my way to pursue the Ph.D. degree. With his beneficent guidance, I have obtained significant improvement in proficiency and confidence as a researcher and educator for my future career. He has made great effort to offer me help in my Ph.D. research in terms of instrument troubleshooting, manuscript revision and presentation practicing. His valuable advice and instruction has ultimately promoted me to be a teaching professor, and furthermore, to achieve my career goals.

I wish to thank my committee members Dr. Glen Jackson, Dr. Stephen Valentine, Dr. Justin Legletier and Dr. Patrick Callery for taking the time to be involved in my $\mathrm{PhD}$ study. I will always be thankful for their insightful discussions, helpful suggestions and valuable feedbacks. Also, I would like to express my gratitude to my fellow group members: Yan Pan, Dr. Han Wang, Dr. Guodong Gu and Dr. Teerapat Rojsajjakul who have given me numerous help and unforgettable friendship.

I would like to make a special acknowledgement to Dr. Harry Finklea for his constant encouragement, thoughtful advice and great inspiration over these years. Thanks to Dr. Michelle Richards-Babb, Dr. Betsy Ratcliff, Dr. Mingming Xu, Mark W. Schraf, Dr. Erin Battin, Dr. Melissa Ely for great mentorship as an educator in another perspective of my graduate study. 
I highly acknowledge the financial support from the Eberly College of Arts and Sciences at WVU and C. Eugene Bennett Chemistry Department at WVU. I wish to thank Allen Burns, Randall Eaglen and Sherman Adams for their professional skills and providing me all the technical supports. Also, thanks to the staff Rebecca Secrist and Brenda Prentiss for warmhearted assistance in my graduate study.

Foremost, I would like to express my sincere appreciation to my parents Jianrong $\mathrm{Xu}$ and Meiying Ou for being my role models and allowing me to realize my own potentials. Their everlasting love and continued support has made all of this possible. 


\section{Table of Contents}

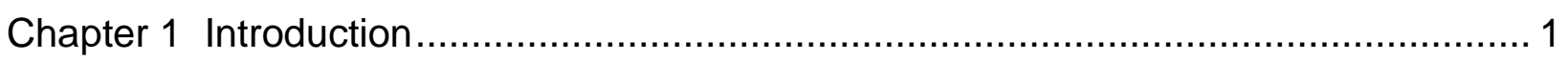

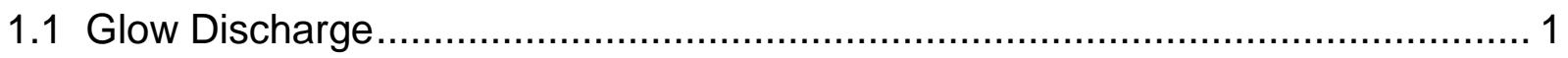

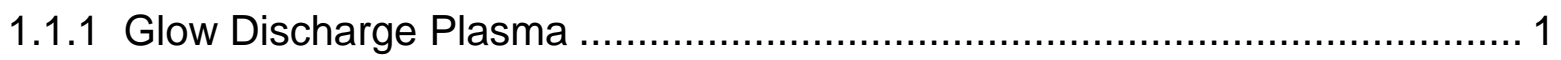

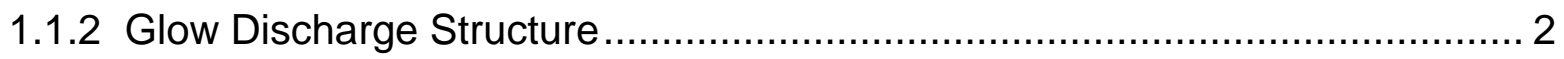

1.1.3 Cathode Dark Space and Cathodic Sputtering …....................................... 3

1.1.4 Negative Glow and Collisional Ionization ............................................... 5

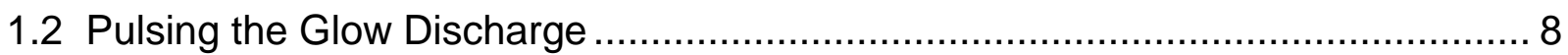

1.2.1 Temporal Regimes in Pulsed Glow Discharge ......................................... 8

1.2.2 Analytical Implications of Temporal Separation of Ionization Mechanisms... 12

1.3 Pulsed Glow Discharge Time-of-Flight Mass Spectrometry ............................. 13

1.4 Application of Glow Discharge Mass Spectrometry for Solution Analysis ........... 18

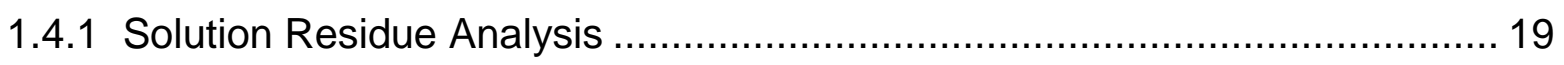

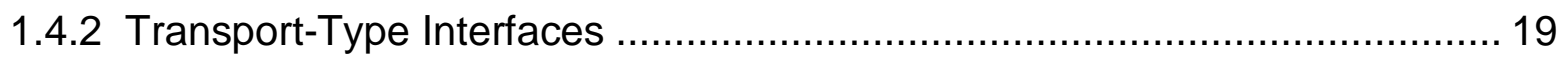

1.5 Application of Glow Discharge Mass Spectrometry in Biological Analysis .......... 21

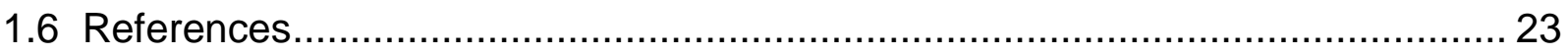

Chapter 2 Analysis of Cysteine Using Pulsed Glow Discharge Time-Of-Flight Mass

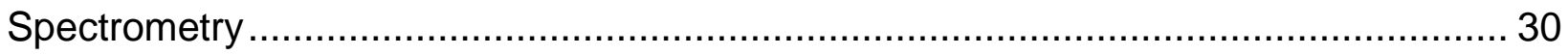

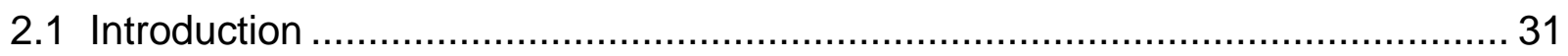

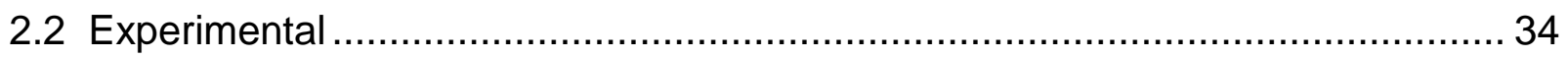

2.2.1 Pulsed Radio Frequency Glow Discharge Time-of-Flight Mass Spectrometry34

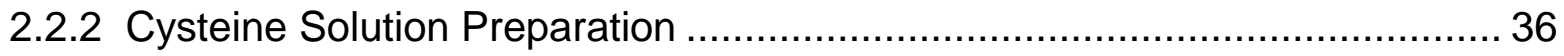

2.2.3 Modifications of Cu Disks for Residue Analysis ....................................... 36

2.2.4 Cysteine Residues Preparation ............................................................ 38 


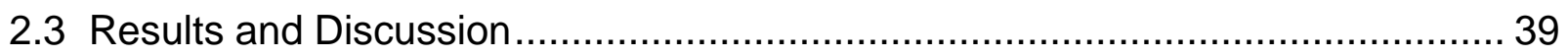

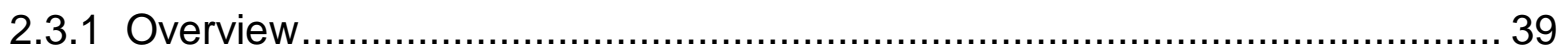

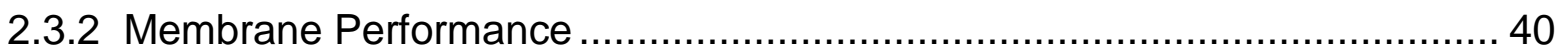

2.3.3 Comparative Study: Cu Reservoir vs Membrane ...................................... 43

2.3.4 Evaluation of GD Operating Parameters ….......................................... 46

2.3.5 Quantification and Reproducibility ..................................................... 49

2.3.6 Comparison with deuterated cysteine $\left(3,3-{ }^{2} \mathrm{H}_{2}\right.$-Cysteine $)$......................... 50

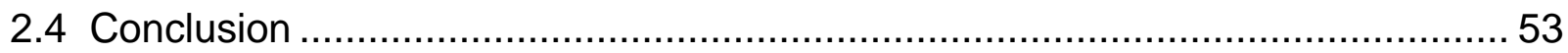

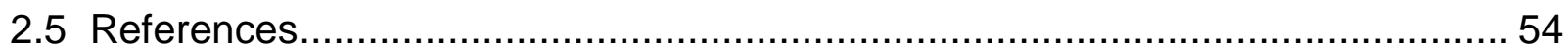

Chapter 3 Extending the Applicability of Pulsed Glow Discharge Mass Spectrometry to

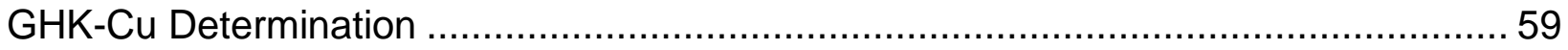

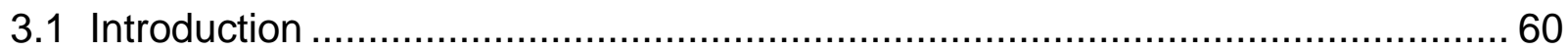

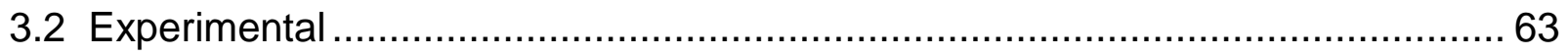

3.2.1 Pulsed Radio Frequency Glow Discharge Time-of-Flight Mass Spectrometry63

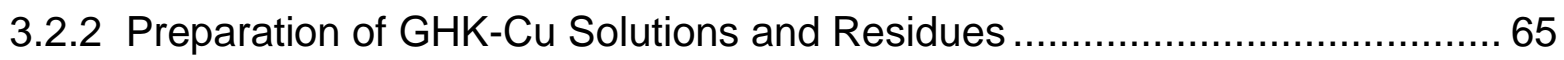

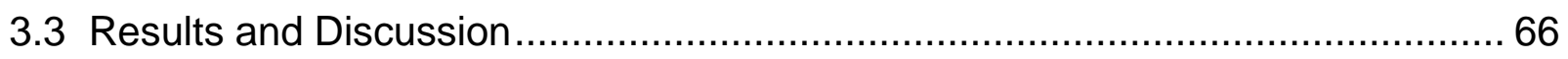

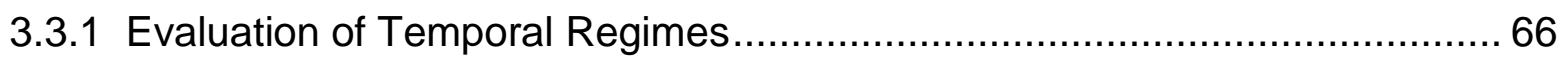

3.3.2 Evaluation of Spatial Variation ............................................................ 71

3.3.3 Optimization of Glow Discharge Operating Parameters ............................ 72

3.3.4 Quantitative Analysis of GHK-Cu Complex.......................................... 77

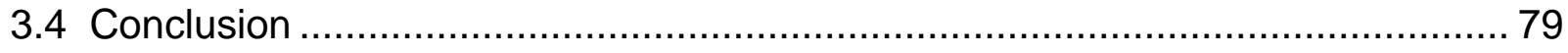

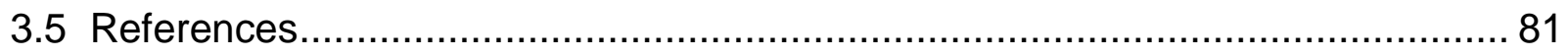

Chapter 4 Glow Discharge Approach for Quantitative Determination of lodinated

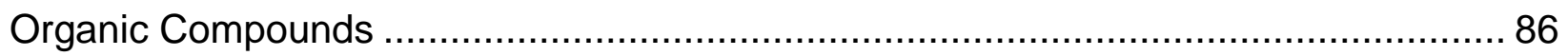

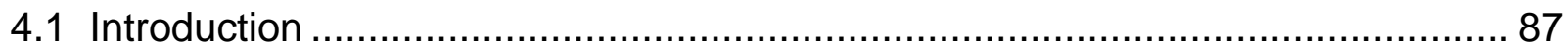

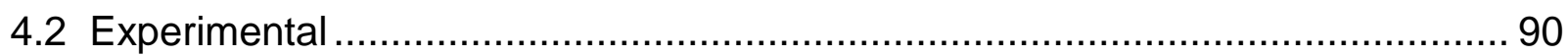


4.2.1 Pulsed Radio Frequency Glow Discharge Time-of-Flight Mass Spectrometry90

4.2.2 Preparation of Thyroid Hormone Solutions and Residues 92

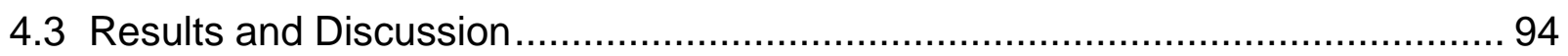

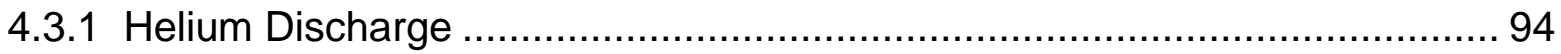

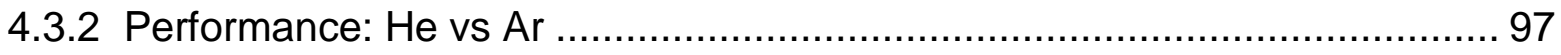

4.3.3 Diiodothyronine (T2) Ion Profiles Over a Pulse Cycle............................. 100

4.3.4 Quantification of Diiodothyronine (T2) …......................................... 102

4.3.5 Comparison among Three Thyroid Hormones........................................ 103

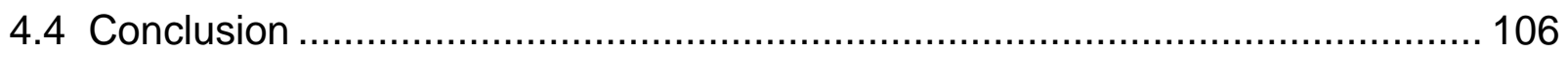

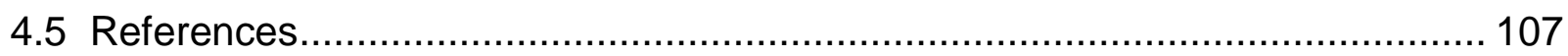




\section{List of Figures}

Figure 1.1 Complete structure of a classic glow discharge. ${ }^{3}$

Figure 1.2 The analytical glow discharge. ${ }^{4}$ 3

Figure 1.3 Sputtering in cathode dark space. Adapted from ref. $6{ }^{6}$ 4

Figure 1.4 Temporal regimes within a pulse cycle. ${ }^{13}$ 9

Figure 1.5 Temporal profiles of (a) Applied voltage (b) Discharge gas species (c) Sputtered species. Adapted from ref.15. ${ }^{15}$ 11

Figure 1.6 Structure of a pulsed glow discharge time-of-flight mass spectrometer. ${ }^{28} . .15$

Figure 1.7 Particle beam interface coupled to glow discharge mass spectrometry. ${ }^{41} . .20$

Figure 2.1 Schematic diagram of 2 modifications of $\mathrm{Cu}$ disks (a) $\mathrm{Cu}$ reservoir (b) Membrane layered Cu disk. 38

Figure 2.2 Mass spectra of (a) Blank Cu reservoir (b) Blank membrane during afterpeak (2.1 ms); GD operating conditions: 400 mTorr discharge pressure, $80 \mathrm{~W}$ RF power, $2 \mathrm{~ms}$ pulse width, $10 \%$ duty cycle, $7 \mathrm{~mm}$ sampling distance.

Figure 2.3 Mass spectra of cysteine on (a) Cu reservoir (b) Membrane during afterpeak (2.1 ms); GD operating conditions: 400 mTorr discharge pressure, $80 \mathrm{~W}$ RF power, 2 ms pulse width, $10 \%$ duty cycle, $7 \mathrm{~mm}$ sampling distance. 43 
Figure 2.4 Cysteine fragment ion intensities for residues prepared on (a) Cu reservoir (b) Membrane; GD operating conditions: 400 mTorr discharge pressure, $80 \mathrm{~W}$ RF power, 2 ms pulse width, $10 \%$ duty cycle, $7 \mathrm{~mm}$ sampling distance. Spectra collected in afterpeak regime $(2.1 \mathrm{~ms})$. Each data point represents averaged intensity from 4 replicates and error bars represent one standard deviation from the mean value.....

Figure 2.5 Evaluation of GD operating conditions influences on cysteine fragment signal (a) Discharge pressure (b) Sampling distance (c) RF power (d) Pulse width (e) Duty cycle; GD operating parameters employed for (a,b,c,e): $70 \mathrm{~W}$ RF power, $2 \mathrm{~ms}$ pulse width, $10 \%$ duty cycle, $400 \mathrm{mTorr}$ discharge pressure, $7 \mathrm{~mm}$ sampling distance except the one being varied for evaluation. For (d), $25 \%$ duty cycle, other parameters are the same. Each data point is the averaged value obtained from 3 measurements and error bars represent one standard deviation from the mean value.

Figure 2.6 Cysteine fragment ion intensities for samples prepared from different concentrations; GD operating conditions: 400 mTorr discharge pressure, $80 \mathrm{~W}$ RF power, 2 ms pulse width, $10 \%$ duty cycle, $7 \mathrm{~mm}$ sampling distance. Spectra collected in afterpeak regime $(2.1 \mathrm{~ms})$. Each data point represents the averaged signal from 2-5 $\min$. 50

Figure 2.7 Structure of deuterated cysteine $\left(3,3-{ }^{2} \mathrm{H}_{2}\right.$-Cysteine $)$ 51

Figure 2.8 Mass spectra of (a) Cysteine (b) Deuterated cysteine during afterpeak (2.1 ms); GD operating conditions: 400 mTorr discharge pressure, 80 W RF power, 2 ms pulse width, $10 \%$ duty cycle, $7 \mathrm{~mm}$ sampling distance. 52 
Figure 3.1 Schematic diagram of the sample introduction. 66

Figure 3.2 Time-resolved mass spectra for Ag cathode (as a blank for comparison) (left) and GHK-Cu residue on silver (right) collected at prepeak (0.3 ms) $(\mathrm{a}, \mathrm{b})$, plateau (1.9 ms) (c, d) and afterpeak (2.3 ms) (e, f). GD operating conditions: 500 mTorr argon discharge pressure, $100 \mathrm{~W}$ RF power, 2 ms pulse width, $10 \%$ duty cycle and $12 \mathrm{~mm}$ sampling distance. 70

Figure 3.3 Spatial effect on ${ }^{63} \mathrm{Cu}^{+}$ion intensity profile collected at $2.35 \mathrm{~ms}$. GD operating conditions: 600 mTorr argon discharge pressure, $100 \mathrm{~W}$ RF power, 2 ms pulse width, $10 \%$ duty cycle. Each data point is the averaged value obtained from 3 replicates and error bars represent one standard deviation from the mean value......... 72

Figure 3.4 Influences of GD operating parameters on ${ }^{63} \mathrm{Cu}^{+}$ion intensity profile: (a) Pressure (b) RF Power (c) Duty cycle (fixed pulsed width $2 \mathrm{ms)}$ (d) Pulse width (fixed duty cycle 10\%). Spectra are collected at $2.35 \mathrm{~ms}$ and $15 \mathrm{~mm}$ sampling distance. GD operating conditions are kept consistent at 600 mTorr argon discharge pressure, 100W RF power, 2 ms pulse width, $10 \%$ duty cycle unless the parameter is varied as $x$-axis. Each data point is the averaged value obtained from 3 replicates and error bars represent one standard deviation from the mean value. 76

Figure $3.5{ }^{63} \mathrm{Cu}^{+}$ion intensity profile over sputtering time for solution residues of $7 \mu \mathrm{L}$. Spectra collected at 2. 35 ms with optimal GD operating conditions: 100 W RF power, 2 ms pulse width, $10 \%$ duty cycle, 600 mTorr argon discharge pressure, and $15 \mathrm{~mm}$ sampling distance. Each data point is the averaged value obtained from 4 replicates and error bars represent one standard deviation from the mean value. 78 
Figure 3.6 Calibration curve of ${ }^{63} \mathrm{Cu}^{+}$ion intensity (collected at 3.75 min from previous figure) vs. GHK-Cu solution concentration. Spectra collected at 2. $35 \mathrm{~ms}$ with optimal GD conditions: $100 \mathrm{~W}$ RF power, 2 ms pulse width, $10 \%$ duty cycle, 600 mTorr argon gas, and $15 \mathrm{~mm}$ sampling distance. Each data point is the averaged value obtained from 4 replicates and error bars represent one standard deviation from the mean value.

Figure 4.1 Global cycle of iodine. ${ }^{5}$ 89

Figure 4.2 Structures of thyroid hormone compounds. 92

Figure 4.3 Breakdown voltage for plane-parallel electrodes for noble gases ${ }^{26}$ 95

Figure 4.4 Afterpeak spectrum (2.1 ms) of $\mathrm{Cu}$ in He plasma GD operating conditions: 2.5 Torr He discharge pressure, 80 W RF power, 2 ms pulse width, $10 \%$ duty cycle and $12 \mathrm{~mm}$ sampling distance. 97

Figure 4.5 Afterpeak spectra $(2.1 \mathrm{~ms})$ of T3 residue in (a) 3 Torr He vs (b) 0.6 Torr Ar Other GD operating parameters: 100 W RF power, 2 ms pulse width, $10 \%$ duty cycle; $17 \mathrm{~mm}$ sampling distance. 99

Figure 4.6 Analysis of T2 over the pulse cycle: (a) Prepeak $0.7 \mathrm{~ms}$ (b) Plateau $1.9 \mathrm{~ms}$ (c) Afterpeak 2.1 ms GD operating conditions: $60 \mathrm{~W}$ RF power, 2 ms pulse width, 10\% duty cycle, $13 \mathrm{~mm}$ sampling distance. 2.5 Torr He discharge gas. 102

Figure 4.7 Calibration curve of diiodothyronine (T2) .......................................... 103 
Figure 4.8 lodine ion intensity over sampling time for TH compounds; GD operating conditions: $70 \mathrm{~W}$ RF power, $2 \mathrm{~ms}$ pulse width, $10 \%$ duty cycle, $18 \mathrm{~mm}$ sampling

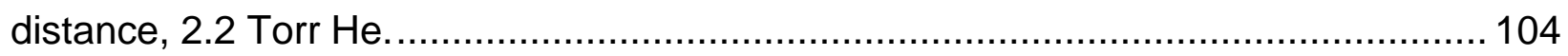

Figure 4.9 Linear relationship among three TH compounds................................. 105 


\section{List of Tables}

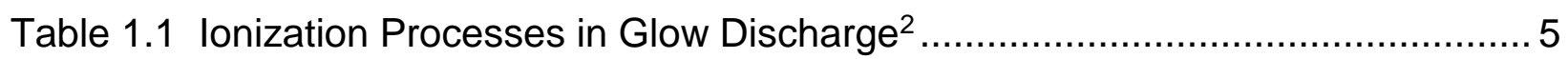

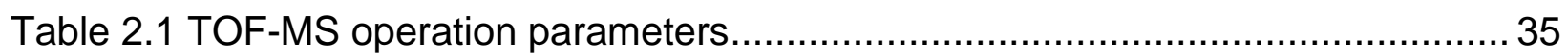

Table 3.1 Pulsed RF GD TOF-MS operating parameters............................... 64

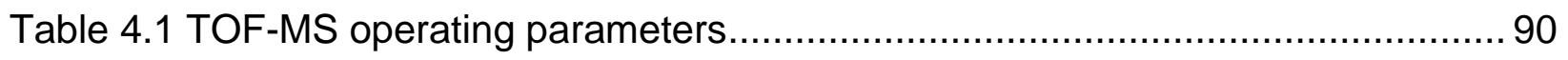




\section{Chapter 1}

\section{Introduction}

\subsection{Glow Discharge}

\subsubsection{Glow Discharge Plasma}

A glow discharge is created when electric current (milliamperes) passes through a gaseous medium. It is usually achieved by applying a potential difference (a few hundred volts) between two electrodes placed at the two ends of a low-pressure cell, filled with noble gas to about $0.1-10$ Torr. ${ }^{1}$ Electrical breakdown of the gas occurs when potential applied to the gaseous medium exceeds the breakdown potential of the gas, which is mainly dependent on the gas identity, density, and interelectrode separation. ${ }^{2}$ Atoms of the gas become ionized via loss of an electron and become charge carriers to conduct electric current. The resulting gaseous mixture is known as a "plasma", consisting of a variety of electrons, excited, ionized and neutral species of the discharge gas, and photons. Argon plasma is most widely used in analytical chemistry. 


\subsubsection{Glow Discharge Structure}

A classic glow discharge (Fig. 1.1) contains eight distinct regions between the electrodes- Aston dark space, cathode layer, cathode dark space, negative glow, Faraday dark space, positive column, anode dark space and anode glow. Most of the analytical utility is found through monitoring of ions within the cathode dark space and the negative glow. ${ }^{2-3}$ Therefore, when creating an analytical glow discharge, the two electrodes are placed close to each other so that the cathode dark space and the negative glow are dominant and other regions are nearly invisible. ${ }^{3}$ A schematic diagram of an analytical glow discharge employed is shown in Fig. 1.2.

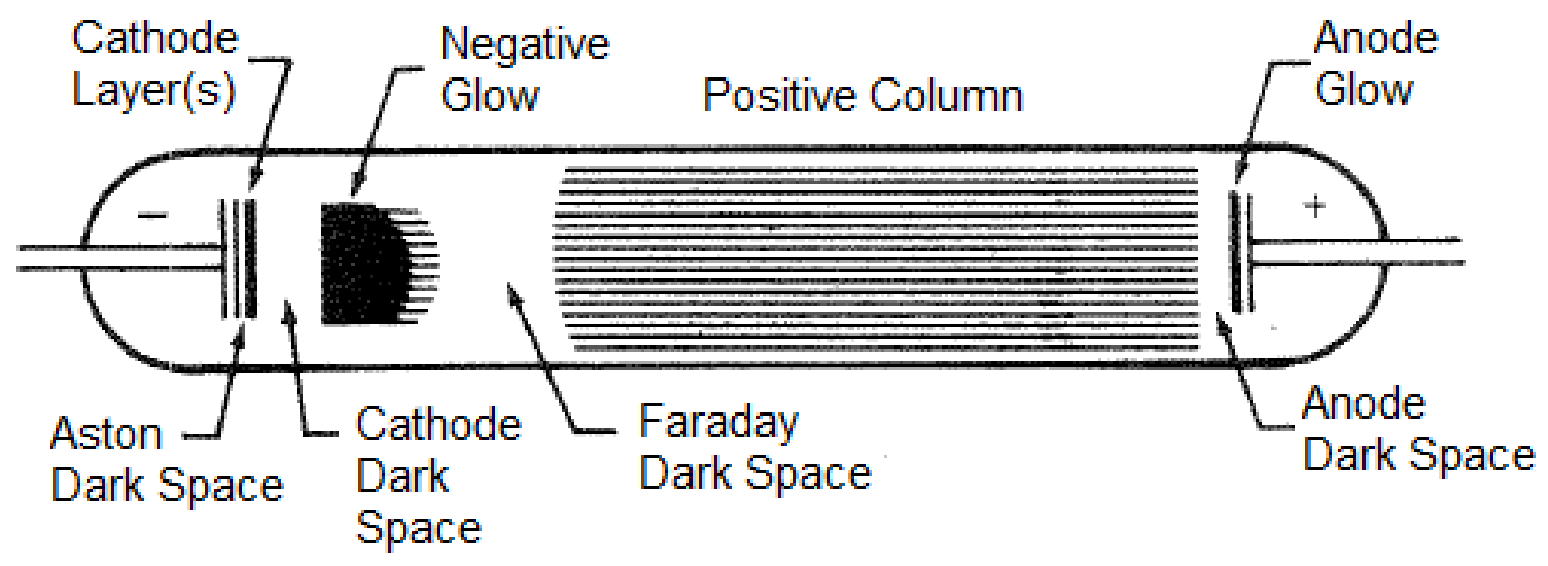

Figure 1.1 Complete structure of a classic glow discharge. ${ }^{3}$ 


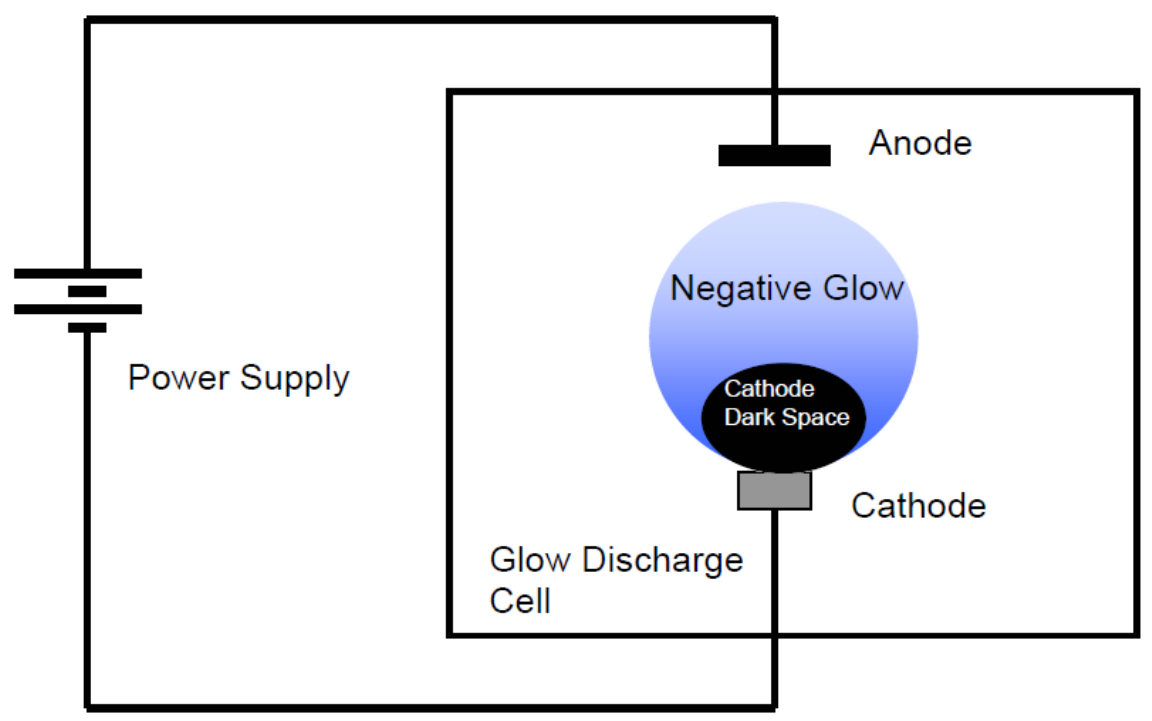

Figure 1.2 The analytical glow discharge. ${ }^{4}$

\subsubsection{Cathode Dark Space and Cathodic Sputtering}

The cathode dark space (CDS), also known as the cathode fall, is a relatively dark region near the cathode where majority of the potential difference between the two electrodes is found. In this region, electrons are repelled away from the cathode with their highest energy, which consequently builds a positive space charge in a narrow region surrounding the cathode. This region maintains a strong negatively biased electric field that accelerates the positive ions of the discharge gas towards the cathode. lons gain kinetic energy traversing though the CDS and impinge the cathode surface resulting in the transfer of momentum and kinetic energy to the cathode material. Consequently, a collision cascade occurs at the cathode surface resulting in emission of secondary electrons, atoms, ions and clusters of the cathode material. This process 
(Fig. 1.3) is called "cathodic sputtering", a significant step that establishes a population of species in the gas phase representative of the cathode material or the sample. ${ }^{5}$ The positive ions and secondary electrons ejected are subject to the effect of the electric field in the vicinity of the cathode, and end up travelling opposite directions in the plasma. lons carrying positive charges are forced to redeposit back to the cathode in this region, whereas electrons will be accelerated through the cathode fall and enter the negative glow with a high kinetic energy, participating in collisions to excite or ionize gaseous species. ${ }^{6}$ The neutral sputtered species that are not affected by the electric field will diffuse through the dark space freely and enter the negative glow. Sputtered cathode species in the negative glow are subject to collisional excitation and ionization that will reveal useful information regarding the cathode material.

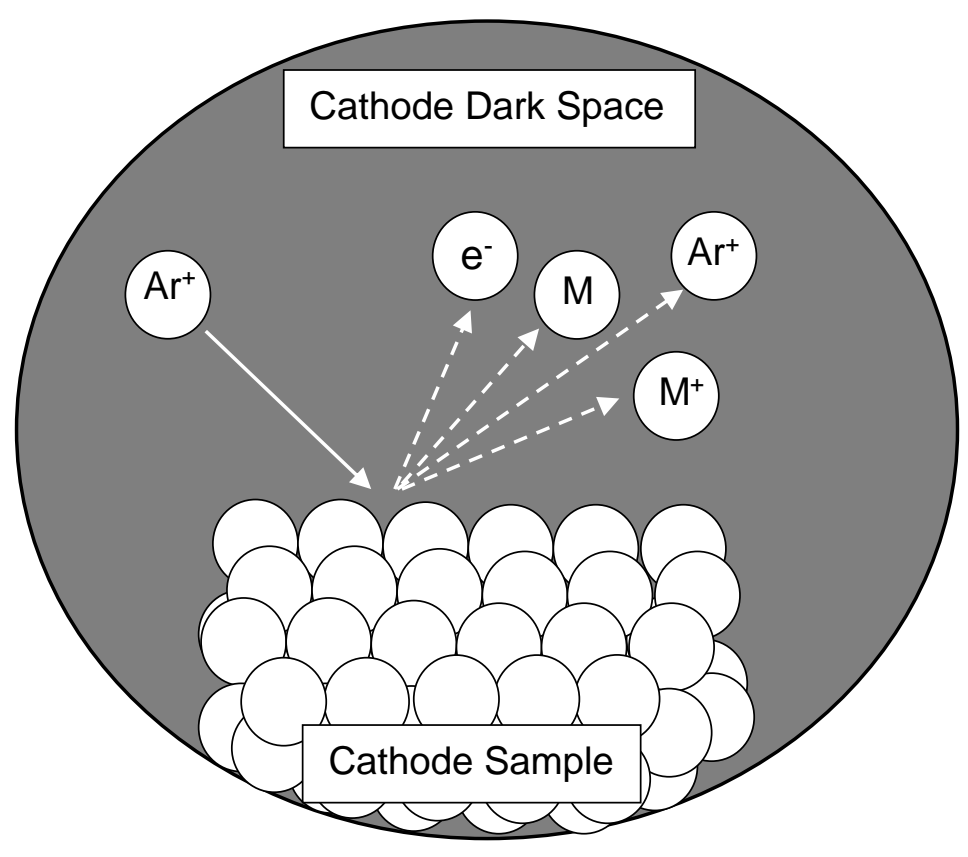

Figure 1.3 Sputtering in cathode dark space. Adapted from ref. $6{ }^{6}$ 


\subsubsection{Negative Glow and Collisional Ionization}

The negative glow (NG) is a highly luminous region adjacent to the CDS. A large number of lower energy electrons and ions exist in this region, making it a collision-rich environment. The population of positive and negative species are equal, so the NG remains relatively field-free as contrast to the strong electric field of the CDS. ${ }^{7}$ Cathode species that have diffused through the CDS will be excited and ionized in this region, providing information that can be observed by optical spectroscopy or mass spectrometry. Because the plasma contains a number of electrons, neutral, excited and ionized discharge gas species, excitation and ionization could be accomplished through collisions with any of these species. Generally, these collision processes (Table 1.1) can be attributed to two types: "collision of the first kind"-collision with light weight electrons; "collision of the second kind"-collision with more massive discharge gas species. ${ }^{8}$

Table 1.1 Ionization Processes in Glow Discharge ${ }^{2}$

Collisions of the first kind

Electron Ionization

$$
\mathrm{A}+\mathrm{e}^{-} \longrightarrow \mathrm{A}^{+}+2 \mathrm{e}^{-}
$$

Collisions of the second kind

Penning lonization

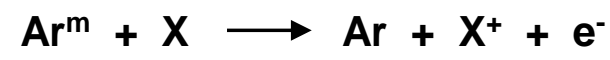

Associative lonization

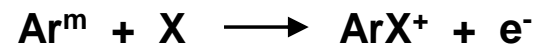

Symmetric Change Exchange

$A^{+}+A \longrightarrow A+A^{+}$

Asymmetric Charge Exchange

$\mathrm{A}^{+}+\mathrm{B} \longrightarrow \mathrm{A}+\mathrm{B}^{+}$ 


\section{Collisions of the First Kind}

Collisions between sputtered species and electrons involve transfer of the kinetic energy from the electrons to the target species, the amount of which determines the nature of the collision induced process occurring in the target. Electrons existing in the negative glow differ in origin and energy. ${ }^{2-3}$ Fast electrons are those accelerated from the CDS and keep an energy above $25 \mathrm{eV}$, secondary electrons created by collisional ionizations have an energy about $7 \mathrm{eV}$, and ultimate electrons are those thermalized by collisions in plasma. Fast and secondary electrons that possess an energy above the ionization potential (IP) of the target species are capable of electron impact ionization. This process can be briefly described as collision between a target atom and an electron that produces a target ion and two slow electrons. One of the two slow electrons is ejected from the target atom, and the other is the cooled primary electron that has lost its energy through collision. In fact, these slow electrons could be involved in another electron impact ionization event providing that their energy exceeds the IP of the target.

Ultimate electrons do not have sufficient energy to effect electron impact ionization, but can contribute to analyte ionization in different way. These thermal electrons can combine with discharge gas ions and produce neutral discharge gas species in excited states. These excited species, therefore, induce another type of collision, "collision of the second kind".

\section{Collisions of the Second Kind}


Collisions between sputtered species and metastable discharge atoms involves a transfer of potential energy rather than kinetic energy. These long-lived or metastable species excite and/or ionize the target species efficiently via the Penning process. In an argon plasma, a metastable argon atom transfers potential energy to the target atom upon collision, producing a neutral argon atom, a target ion and an electron. Because of the long lifetime of metastable species, Penning ionization has been identified as the dominant ionization process of the sputtered species in many glow discharge sources. ${ }^{9-}$ 10 Metastable argon atoms well serve as Penning ionizing reagents. Because their potential energy (11.5 and $11.7 \mathrm{eV}$ ) is above the first IPs, but below the second IPs of most elements. Therefore, collision with metastable argon species would maximize the production of singly charged species by removing only one electron.

Collisions between sputtered species and the discharge gas ions is another important process that produces analyte ionization involving a transfer of potential energy. Sputtered target atoms can be ionized via an asymmetric charge exchange process with the discharge ions. Comparing to electron impact and Penning ionization that ionization will occur as long as the target's IP is met, but is more favored the closer the IP's of the two collision partners. This is because the resonant nature of the charge exchange process. When argon is used as discharge gas, charge exchange between an argon ion and a target atom will only occur when relaxation of the argon ion to a neutral state releases an energy in very close to that required to bring the neutral target atom to an ionized level. This is a highly selective process, as a result, charge exchange is less likely to be the major contributor to sample ionization in GDs. ${ }^{2}$ 


\subsection{Pulsing the Glow Discharge}

GDs can be operated in either continuous or pulsed power modes. In the continuous mode, power is constantly applied to sustain the plasma, whereas in the pulsed mode, there exists power-on and power-off periods. Pulsed power is usually described by three parameters including pulse power, pulse width, and repetition frequency. A typical pulse parameter used in this study is a $10 \%$ duty cycle consisting of a $2 \mathrm{~ms}$ power on period followed by a $18 \mathrm{~ms}$ power off period. The use of a pulsed plasma offers a number of advantages that are not afforded by a continuous glow discharge. First, it provides an efficient way to supply higher instantaneous power and produce enhanced sputter yields and detection limits. Continuous GDs cannot employ high power to enhance analysis due to potential sample overheating problems. Pulsed GDs provide a solution to this problem by supplying high power for a short period and then allowing sample to cool in the rest of the power cycle. Studies by Harrison has shown that in a $50 \%$ duty cycle, the effective voltage of a pulsed discharge can be increased to $40-60 \%$ above the voltage applied in a continuous GD to maintain the same average current. ${ }^{11}$ Sputter yield enhanced by a two orders of magnitude was reported. ${ }^{12}$ Secondly it affords access to temporal regions that have different ionization/excitation characteristics as described below.

\subsubsection{Temporal Regimes in Pulsed Glow Discharge}

The pulsed GD provides three temporal plasma domains within the power cycle. When a pulse is first applied to initiate the discharge, the plasma does not reach a 
steady state instantaneously. Similarly, when power is removed, the plasma species does not disappear immediately. Therefore, a pulsed discharge creates a timedependent plasma that differs in constituents and their relative amounts. Sputtered species entering the plasma in different time domains would experience distinct excitation and ionization processes. To fully understand the utilities of a pulsed glow discharge, it is necessary to investigate the different temporal regimes of the plasma, termed: prepeak, plateau, afterpeak (Fig. 1.4).

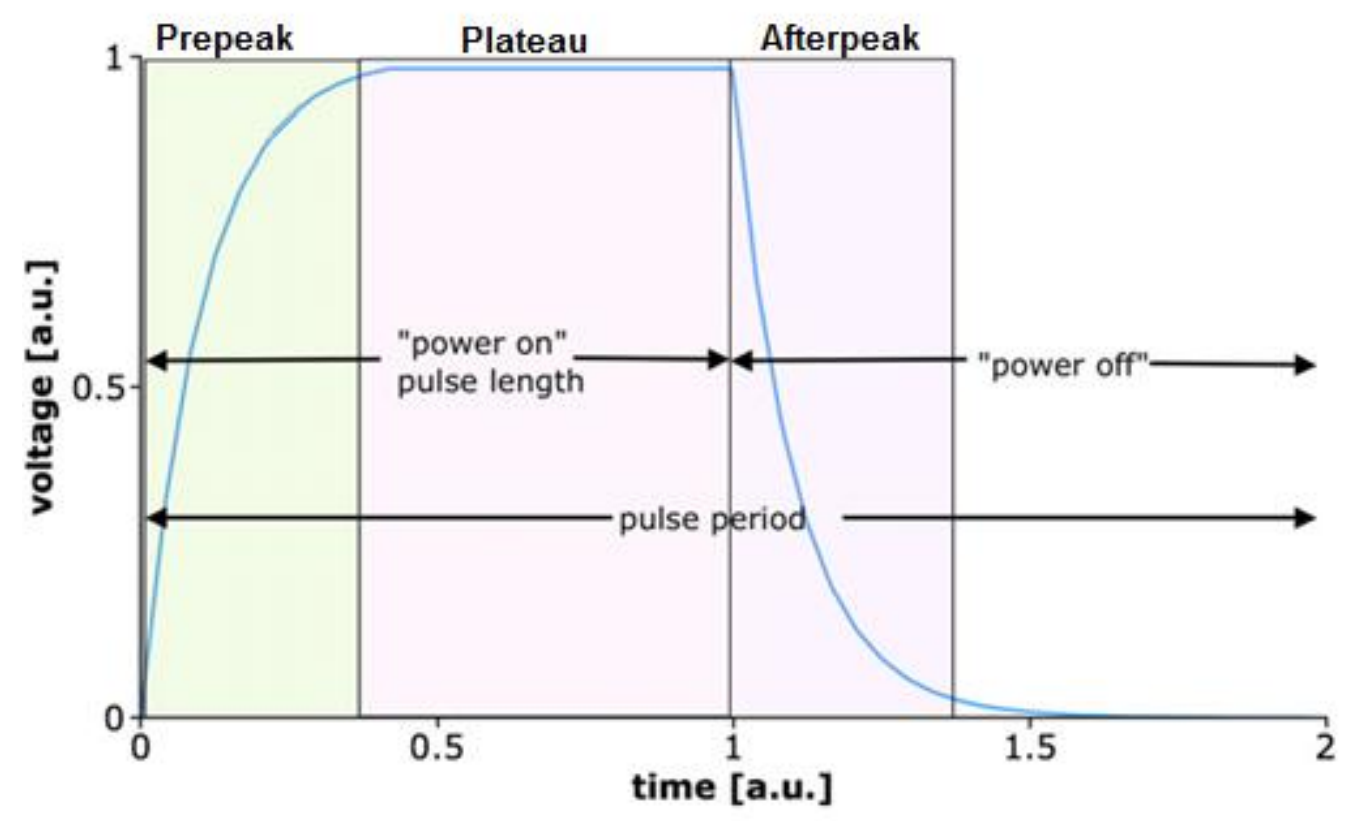

Figure 1.4 Temporal regimes within a pulse cycle. ${ }^{13}$

Prepeak When power is turned on, breakdown of the discharge gas occurs and produces discharge gas ions and electrons. The electrons collide and ionize the gaseous species within the plasma, mostly the discharge gas atoms, resulting in a 
surge of the population of discharge gas ions (Fig. 1.5). This time regime is termed as "prepeak", when electron ionization of the discharge gas is the dominant process within the plasma. Ion signals of the sputtered species are absent because the sample sputtering, diffusing and subsequent ionization does not occur immediately. Spectra collected in this regime consists of ion signals of the discharge gas species and atmospheric contaminants. ${ }^{14-16}$

Plateau Shortly after the plasma initiation, the ion signals of gas species start to decline and ion signals of sputtered species arise (Fig. 1.5). These ionized sputtered species soon reach ay stable population in the plasma that persists until the power is removed. This period is called "plateau", during which the plasma environment resembles that of a continuous discharge. Energetic electrons, argon ions, metastable argon atoms coexist in the plasma and provide collisional ionization via a mixture of electron impact, Penning ionization and charge exchange processes. ${ }^{16}$

Afterpeak This regime represents a transient period in the plasma process beginning with power removal and extending to the complete extinction of plasma (Fig. 1.5). With no power sustaining the discharge, plasma stops the production of electron-ion pairs. No energetic electrons can be produced since the electric field is removed. Therefore, electron impact ionization of the discharge gas halts immediately after power termination. Ion signals of the discharge gas species were observed to decay rapidly in the afterglow. ${ }^{11,15-17}$ The remaining fast electrons in plasma quickly thermalize through collisions with other plasma species. Recombination between the thermalized electrons and the discharge gas ions is encouraged, producing a large population of metastable 
discharge species in the afterpeak. This recombination process supplies a large number of Penning reagents, and, as a result, Penning ionization becomes the dominant ionization process in the afterpeak. Sputtered species remaining in plasma undergo efficient Penning ionization through collisions with these metastable discharge species. A sudden increase of the sputtered species ion signal was observed shortly after power removal. ${ }^{11,14,16}$

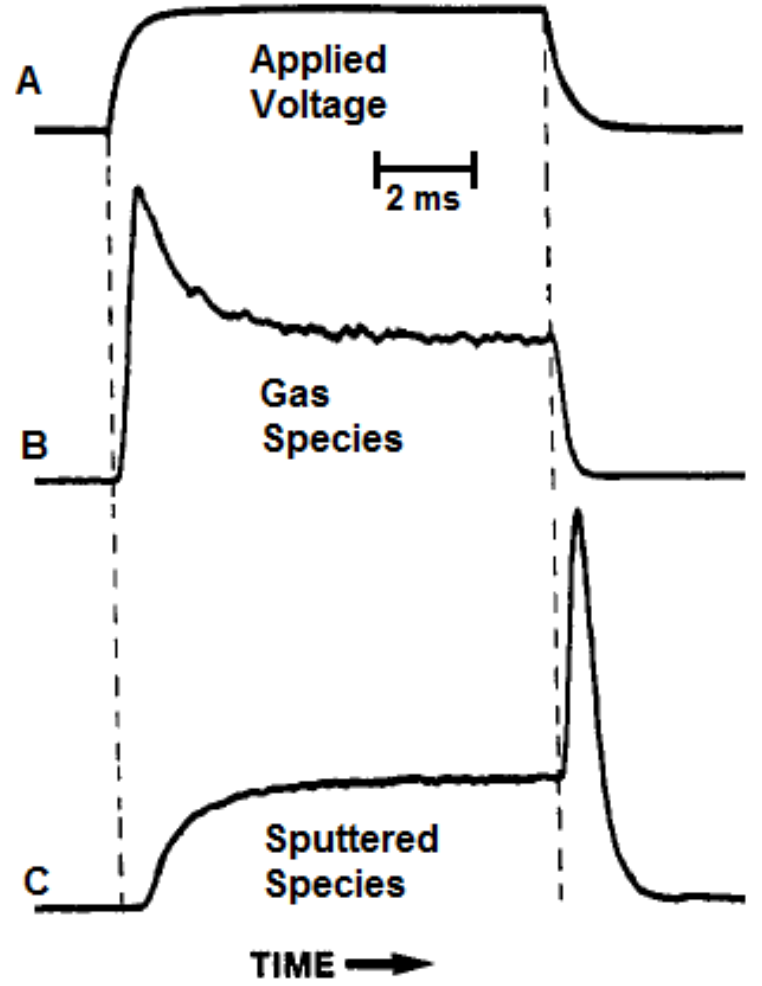

Figure 1.5 Temporal profiles of (a) Applied voltage (b) Discharge gas species (c) Sputtered species. Adapted from ref. $15 .^{15}$ 


\subsubsection{Analytical Implications of Temporal Separation of Ionization}

\section{Mechanisms}

Overall, pulsing the GD allows the temporal separation of different ionization mechanisms. Analysis accomplished in each stage of a pulsed plasma should allow one to acquire unique information regarding the elemental, structural and molecular constituents of a given sample. This advantage is particularly obvious when gaseous analytes are analyzed by GDMS. A number of studies have utilized this feature of pulsed glow discharge and achieved quasi-simultaneous elemental and molecular analysis of multiple compounds. ${ }^{18-21}$ In the prepeak, elemental and structural information caused by energetic fragmentation can be obtained, resembling the characteristic signals obtained with electron impact ionization, whereas intact molecular ions were found in the afterpeak produced by soft Penning ionization. This convertibility of plasma energetics from "hard" to "soft" over a pulse cycle can be clearly seen in a study of its internal energy distribution ${ }^{22}$ and has found previous applications in chemical speciation. ${ }^{23-25}$

In addition, pulsing the glow discharge adds the possibility to perform spectral discrimination against the background and contaminant species. As previously discussed, ion profiles of the discharge gas and sputtered species display strong temporal dependence over the pulse cycle in a pulsed plasma. This differential behavior permits the use of time-gated detection to collect analyte signals at appropriate time windows along the cycle with minimized spectral interferences. It has been shown 
that distinct analyte ion signals can be recorded in afterpeak without potential interferences that exist in other portion of a pulsed plasma. ${ }^{26-27}$

\subsection{Pulsed Glow Discharge Time-of-Flight Mass Spectrometry}

To fully exploit the benefits of a pulsed glow discharge source, it must be coupled with a detection system capable of time-gated acquisition. The time-of-flight is a simple mass analyzer that differentiates mass-to-charge ratios of ions based on their flight time across a fixed distance. The time-of-flight mass spectrometer is an ideal mass analyzer for a pulsed glow discharge ion source for several reasons. ${ }^{28-29}$ First and most importantly, it uses pulsed injection of an ion packet. This intrinsic feature makes it a perfect match for sampling a glow discharge source in pulsed operation. Second, it allows simultaneous detection of all ions generated at the same time, so a complete spectrum can be generated within every pulse. This cannot be achieved with quadrupole or sector mass analyzers. ${ }^{30}$ In addition, the time-of-flight mass spectrometer has a high repetition rate as well as efficient ion transmission. It only takes tens of microsecond for an ion of a high mass (up to $\mathrm{m} / \mathrm{z} 1000$ ) to travel through a flight tube of $1 \mathrm{~m}$. So, collection of a complete mass spectrum is very fast. This indicates that a large number of mass spectra can be obtained, averaged and integrated within a short time scale, resulting in enhanced signal/noise ratio and detection limits.

The schematic diagram of the home build glow discharge time-of-flight (GD-TOF) mass spectrometer used in this work is shown in Fig. 1.6. The Time-of-flight mass 
analyzer is positioned orthogonally to the direction of the ion beam generated in GD source. This orientation is employed to minimize the kinetic energy dispersion of ions in the flight tube direction ( $\mathbf{Z}$ direction). The detector is a dual microchannel plate and located at the end of the flight tube. It converts the ion signals to electron currents and easily amplifies the ion signal by a magnitude of $10^{6}$. After amplification, the analog signal is fed into an oscilloscope to produce a temporal ion signal profile. Data is then transferred to a computer through a GPIB interface and processed by Microsoft Excel to generate a mass spectrum.

This GD-TOF mass spectrometer is composed of several compartments that function at different pressures. The glow discharge chamber works at a reduced pressure of 0.1-10 Torr when filled with discharge gas, but the mass analyzer and the detector function under high vacuum at a pressure of $10^{-5}-10^{-9}$ Torr. Therefore, differential pumping by two mechanical pumps and two turbomolecular pumps is incorporated to maintain the pressures required for different parts of the system and allow the instrument to properly function.

lons generated in the plasma exit the glow discharge chamber through an orifice located on the center of a back plate. These ions are then extracted by the skimmer and a slit sitting behind the skimmer to limit the spatial dispersion of ions in the $\mathbf{X}$ direction. After that, ions reach the extraction region of the TOF which is defined by a repeller plate (A1) and a grounded grid (A2). When a positive pulse (of adjustable width) is applied to the repeller plate (A1), ions in the extraction region will be injected and deflected to the flight tube direction ( $\mathbf{Z}$ direction). The repeller pulse is set at a selected 
delay with respect to the GD pulse. By adjusting the delay, one can easily select the ions formed at a desired time within the pulse cycle to be injected to the mass analyzer. These ions will first pass a strong electric field between the grounded and accelerator grids and accelerate to velocities that are a function of their mass and the accelerating voltage.

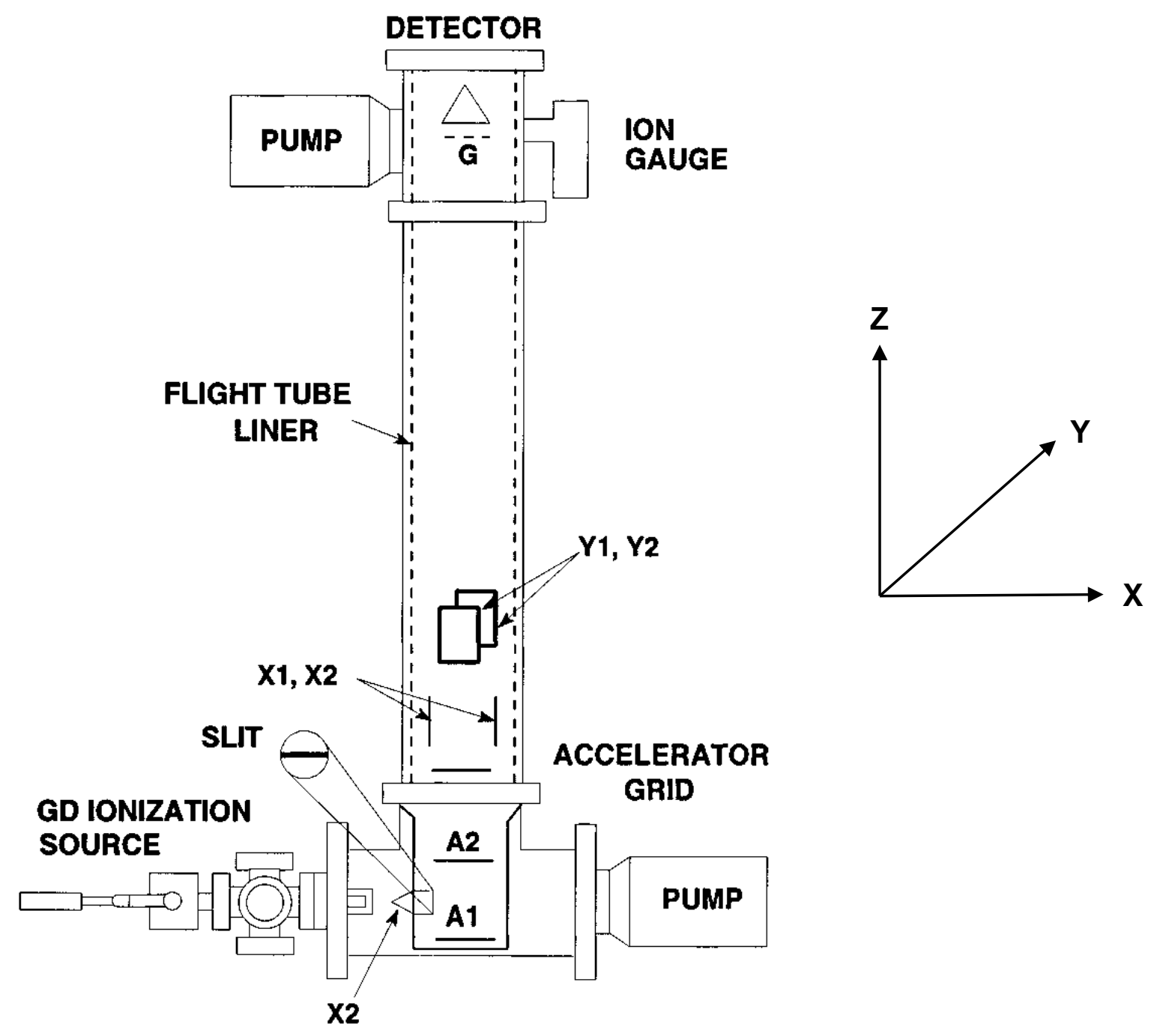

Figure 1.6 Structure of a pulsed glow discharge time-of-flight mass spectrometer ${ }^{28}$ 
Assuming a negative potential $(\mathrm{V})$ is applied to the accelerator grid, an ion of a mass $(m)$ and charge (z) acquires potential energy (Ep) after travelling through the electric field:

$$
E_{p}=z e V
$$

where $\mathbf{e}$ is the charge on an electron. The potential energy gained is expressed as the kinetic energy of the ion, which is a function of mass $(\mathbf{m})$ and velocity $(\mathbf{v})$ :

$$
z e V=E_{p}=E_{k}=\frac{1}{2} m v^{2}
$$

Rearrange the equation and solve for $\mathbf{v}$ :

$$
V=\left(\frac{2 e V}{m / z}\right)^{1 / 2}
$$

From this equation, it is clear that the velocity of an ion after acceleration is inversely proportional to the square root of its mass-to-charge ratio $(\mathbf{m} / \mathbf{z})$. So, ions of different $m / z$ values will enter the flight tube at different velocities. Ions of a smaller $\mathrm{m} / \mathrm{z}$ will have a higher velocity. Because the flight tube is maintained under high vacuum and is field-free in the direction of ion path, ions are assumed to travel through the drift region at a constant velocity. The time $(\mathbf{t})$ required for the ion to drift through the tube of a length of $(\mathbf{L})$ is 


$$
t=\frac{L}{v}
$$

Replace the $\mathbf{v}$ with above equation and find:

$$
t=\left(\frac{m}{z}\right)^{1 / 2}\left(\frac{L^{2}}{2 e V}\right)^{1 / 2}
$$

In the above equation, $\mathbf{L}$ is fixed, $\mathbf{e}$ is a universal constant, $\mathbf{V}$ is the accelerating voltage that applies to all ions. It is obvious that flight time (t) is proportional to the square root of $\mathbf{m} / \mathbf{z}$. Ions of a smaller $\mathbf{m} / \mathbf{z}$ will reach the detector faster than ions of a higher $\boldsymbol{m} / \mathbf{z}^{31}$

For example, the TOF used in this lab has a flight tube of $1 \mathrm{~m}$ and accelerating voltage of $1805 \mathrm{~V}$. The time for a ${ }^{63} \mathrm{Cu}^{+}$to reach the detector is

$$
t=\left(\frac{63 *\left(1.66 * 10^{-27} k g\right)}{1}\right)^{1 / 2}\left(\frac{(1 m)^{2}}{2 *\left(1.602 * 10^{-19} C\right) *(1805 V)}\right)^{1 / 2}
$$

$$
=13 \mu \mathrm{s}
$$

Similarly, one can calculate that an ion of $\mathrm{m} / \mathrm{z} 1000$ only takes $54 \mu$ s to pass through the flight tube. Since all ions are injected into the flight tube and analyzed 
simultaneously, a spectrum of a wide $\mathrm{m} / \mathrm{z}$ range can be generated within tens of microseconds, same as the flight time of the ion having the highest $\mathrm{m} / \mathrm{z}$ value.

\subsection{Application of Glow Discharge Mass Spectrometry for}

\section{Solution Analysis}

The glow discharge is well recognized for its ability to analyze materials directly in the solid state. Direct current (DC) powered GDs readily analyze conducting solids, while radio frequency $(\mathrm{RF})$ powered GDs further extend its application to direct analysis

of semiconductors and nonconducting solids. ${ }^{32-33}$ However, GDs are not widely applied to solution analysis. This limited application is associated with solvent-related problems. Generally, the GD plasma is insufficient to produce complete desolvation of solution samples. The GD operates at low gas density and temperature, ${ }^{2-3}$ so it does not have sufficient energy to completely evaporate the solvent. As a result, cathodic sputtering that is expected to release the analyte species as isolated atoms/molecules will be suppressed. Even if sufficient desolvation is attainable to produce dry analyte particles, another solvent-relevant problem would arise. The solvent vapors in the GD would quench the plasma energetics by interfering in the gas-phase collisional ionization processes, leading to a potential failure in acquisition of analyte information. ${ }^{34}$ Therefore, efforts were made to completely desolvate the solution sample prior to the introduction of analyte into the GD plasma. Different approaches were developed to isolate analyte from solvent, with the two most important methods being analysis of dry solution residue and the use of transport-type interfaces. 


\subsubsection{Solution Residue Analysis}

Preparation of solution residues is the most widely applied method in GD analysis of liquids. To prepare dry residues, analyte solution is deposited in or on the cathode of the glow discharge. Solution added onto the cathode surface is directly dried to form a thin film of analyte residue, whereas sometimes analyte solution is added to a conducting host matrix and then the dried mixture is blended and compacted

into a homogenous pellet serving as GD cathode. ${ }^{35-36}$ Analyte prepared in either way is then introduced to a GD source and normal cathodic sputtering is applied to generate analyte population in the gas phase for subsequent mass spectrometric analysis. The use of a host cathode to support solution residue sets high requirements for the cathode material. It should have a high sputtering rate to benefit analyte sputtering as well as high purity to avoid background interferences to analyte signals. ${ }^{34}$

\subsubsection{Transport-Type Interfaces}

The above method only applies to solution samples of a small volume, and is not suitable for the analysis of continuous liquid chromatography (LC) eluents in a GD source. On-line coupling of LC to GD source would face the same problems encountered in coupling LC with any other low-pressure ion sources, like $\mathrm{El}$ and $\mathrm{Cl}$. Therefore, a transport-type interface, such as a moving belt ${ }^{37-38}$ and particle beam interface $^{39-40}$ that were employed in conventional LC-MS coupling, have been implemented to deliver solvent-free analyte to GD sources. In general, such an interface includes compartments of on-line sampling, desolvation, solvent vapor 
removal, and analyte delivery to the GD plasma. ${ }^{34} \mathrm{~A}$ more widely used particle beam interface (Fig. 1.7) will be discussed here about its function mechanism.

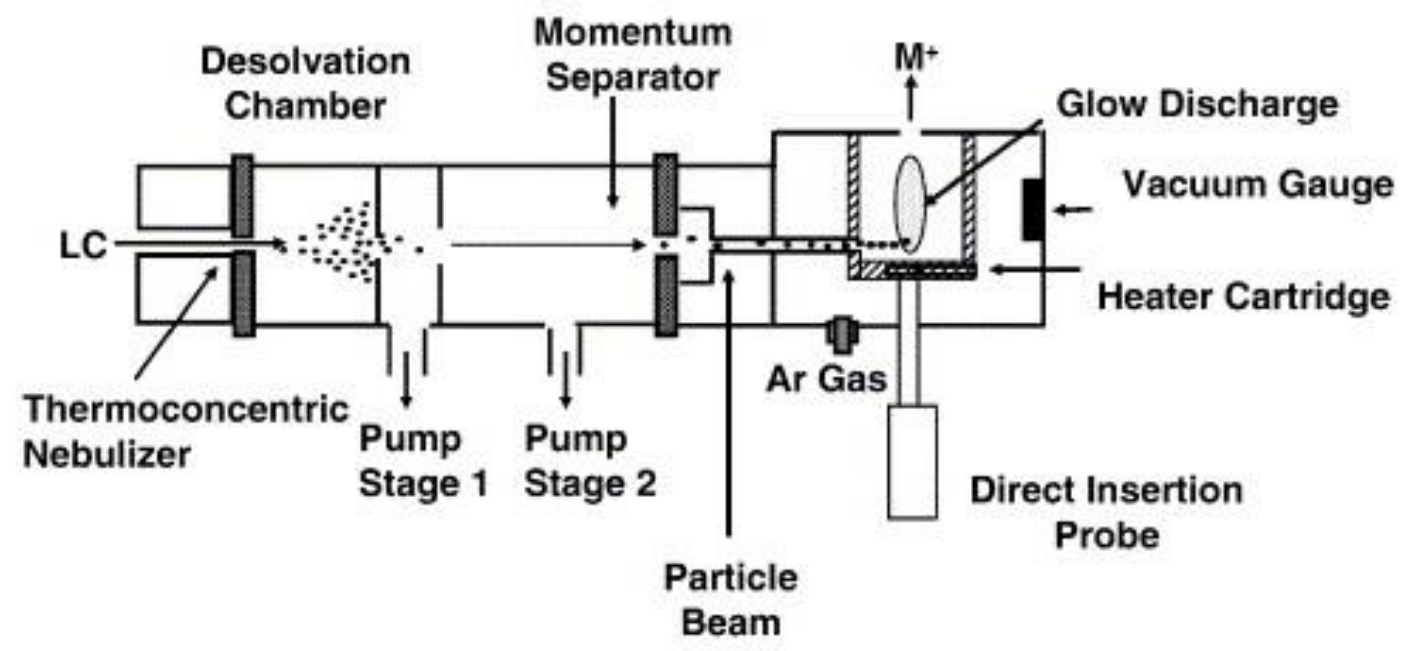

Figure 1.7 Particle beam interface coupled to glow discharge mass spectrometry. ${ }^{41}$

Eluent coming out of LC separation column will first pass a nebulizer located in a heated chamber, and the aerosol produced will undergo desolvation. The resulting mixture containing solvent vapor, nebulizer gas and analyte species will subsequently pass through a set of successive orifices across which pressure differentials are placed. When diffusing through the orifices, the mixture components undergo expansions to different degrees. A high degree of expansion will occur to the light solvent and the nebulizer gas, where the massive analyte is more likely to stay focused as a beam. Based on this momentum difference, analyte particle can be separated from the solvent and the nebulizer gas and eventually reach the ion source. Application of a particle 
beam interface to coupling of LC to GD has offered combined advantages of high separation power, efficient solvent removal, as well as sensitive and flexible detection. ${ }^{34}$,

Nowadays, direct solution analysis has been achieved with glow discharge sources that operate at ambient pressure. ${ }^{42-43}$ This type of source employs the analyte solution as the cathode and establish an abnormal glow discharge between the solution cathode and a metal electrode to perform analysis. Since first introduced in $1993,{ }^{44-45}$ a variety of ambient glow discharge sources has been developed ${ }^{46-48}$ and applied to the mass spectrometric analysis of diverse solutions. ${ }^{49-51}$

\subsection{Application of Glow Discharge Mass Spectrometry in}

\section{Biological Analysis}

The most common technique for quantitative bioanalysis is liquid chromatography - tandem mass spectrometry (LC-MS/MS) employing a soft ionization source, such as ESI/MALDI. ${ }^{52}$ Peptide/protein quantification is usually achieved with transitions from selected precursor ions to characteristic fragment ions using multiple reaction monitoring technique. In order to realize absolute quantification, the use of isotope-labelled peptides as internal standards is required, making the entire analysis expensive and time consuming. In contrast, elemental mass spectrometry, particularly inductively coupled plasma-mass spectrometry (ICP-MS), has become a significant complementary technique found wide applications in routine bioanalysis. ${ }^{53}$ Natural occurring heteroelements, such as sulfur, ${ }^{54}$ phosphorus, ${ }^{55}$ selenium, ${ }^{56}$ and metals ${ }^{57}$ in a 
peptide/protein with a known stoichiometry allow peptide quantification to be completed with the measurement of one single element. Absolute quantification is accomplished via the post-column isotope dilution technique with the addition of an enriched isotope of the element concerned, avoiding the preparation of peptide standards required in other methods. Furthermore, the development of element labelling/tagging strategies extend the application of ICP-MS to the quantitative analysis of almost every protein or peptide. ${ }^{58-59}$ However, molecular information is lost in ICP-MS.

Pulsed glow discharge mass spectrometry is a powerful tool that has combined advantages of elemental and molecular mass spectrometry. In a glow discharge pulse cycle, a plasma quickly evolves from energetic environment (dominated by fast electrons, prepeak) upon power initiation to soft environment (dominated by metastable discharge atoms, afterpeak) after power termination. Gaseous analyte introduced to such a plasma will undergo fragmentation to the different levels as the plasma components constantly change. The prepeak regime can be used to obtain abundant elemental information, the plateau regime is best used to collect structural information, and the afterpeak regime will produce ions of intact molecules or large fragments. The use of GDMS as a detector for gas chromatography (GC) has demonstrated its capability in concurrent acquisition of elemental, structural and molecular information. ${ }^{24}$, 60 Coupling with a separation technique, GDMS is a promising tool for simultaneous collection of both qualitative and quantitative information in the analysis of biological species. The work here explores the capability of GDMS in analysis of biological samples on the quantitative aspect. 


\subsection{References}

1. Harrison, W. W.; Hess, K. R.; Marcus, R. K.; King, F. L., Glow discharge mass spectrometry. Anal. Chem. 1986, 58 (2), 341A-356A.

2. Marcus, R. K., Glow discharge spectroscopies. Springer Science \& Business Media: 2013.

3. Chapman, B. N., Glow discharge processes: sputtering and plasma etching. Wiley: 1980.

4. Zhang, N., Elemental speciation using pulsed Glow Discharge Time-of-Flight Mass Spectrometry. West Virginia University: 2009.

5. Westwood, W. D., Glow discharge sputtering. Prog. Surf. Sci. 1976, 7 (2), 71-111.

6. King, F. L.; Harrison, W. W., Glow discharge mass spectrometry: An introduction to the technique and its utility. Mass Spectrom. Rev. 1990, 9 (3), 285-317.

7. Fang, D.; Marcus, R. K., Use of a cylindrical Langmuir probe for the characterization of charged particle populations in a planar, diode glow discharge device. Spectrochimica Acta Part B: Atomic Spectroscopy 1990, 45 (9), 1053-1074.

8. Nasser, E., Fundamentals of gaseous ionization and plasma electronics. WileyInterscience: 1971.

9. Hess, K. R.; Harrison, W. W., The role of metastable atoms in glow discharge ionization processes. Anal. Chem. 1988, 60 (7), 691-696.

10. Smith, R. L.; Serxner, D.; Hess, K. R., Assessment of the relative role of Penning ionization in low-pressure glow discharges. Anal. Chem. 1989, 61 (10), 1103-1108.

11. Klingler, J. A.; Savickas, P. J.; Harrison, W. W., The pulsed glow discharge as an elemental ion source. J. Am. Soc. Mass. Spectrom. 1990, 1 (2), 138-43.

12. Pollmann, D.; Ingeneri, K.; Harrison, W. W., Comparison of atomization and ionization processes in direct current, radiofrequency and microsecond pulse discharges. J. Anal. At. Spectrom. 1996, 11 (9), 849-853. 
13. Belenguer, P.; Ganciu, M.; Guillot, P.; Nelis, T., Pulsed glow discharges for analytical applications. Spectrochimica Acta Part B: Atomic Spectroscopy 2009, 64 (7), 623-641.

14. Pan, C.; King, F. L., Time-resolved studies of ionized sputtered atoms in pulsed radio frequency powered glow discharge mass spectrometry. Anal. Chem. 1993, 65 (22), 3187-3193.

15. Klingler, J. A.; Barshick, C. M.; Harrison, W. W., Factors influencing ion signal profiles in pulsed glow discharge mass spectrometry. Anal. Chem. 1991, 63 (22), 2571-2576.

16. DeJesus, M. R.; Gu, G.; King, F. L.; Barnes, J. H.; Lewis, C. L., Ion formation in millisecond pulsed glow discharge plasmas. J. Anal. At. Spectrom. 2011, 26 (11), 2206-2215.

17. King, F. L.; Pan, C., Temporal signal profiles of analytical species in modulated glow discharge plasmas. Anal. Chem. 1993, 65 (6), 735-739.

18. E. Steiner, R.; L. Lewis, C.; Majidi, V., Consideration of a millisecond pulsed glow discharge time-of-flight mass spectrometer for concurrent elemental and molecular analysis. J. Anal. At. Spectrom. 1999, 14 (9), 1537-1541.

19. Tarik, M.; Gunther, D., A laser ablation millisecond-pulsed glow discharge time-of flight mass spectrometer (LA-GD-TOFMS) for quasi-simultaneous elemental and molecular analysis. J. Anal. At. Spectrom. 2010, 25 (9), 1416-1423.

20. Lotito, G.; Günther, D., Tunable fragmentation of organic molecules in laser ablation glow discharge time-of-flight mass spectrometry. Analytical and bioanalytical chemistry 2012, 402 (8), 2565-2576.

21. Solà-Vázquez, A.; Martín, A.; Costa-Fernández, J. M.; Pereiro, R.; Sanz-Medel, A., Tuneable Microsecond-Pulsed Glow Discharge Design for the Simultaneous Acquisition of Elemental and Molecular Chemical Information Using a Time-of-Flight Mass Spectrometer. Anal. Chem. 2009, 81 (7), 2591-2599.

22. Li, L.; Millay, J. T.; Turner, J. P.; King, F. L., Millisecond pulsed radio frequency glow discharge time of flight mass spectrometry: temporal and spatial variations in molecular energetics. J. Am. Soc. Mass. Spectrom. 2004, 15 (1), 87-102. 
23. Majidi, V.; Moser, M.; Lewis, C.; Hang, W.; King, F. L., Explicit chemical speciation by microsecond pulsed glow discharge time-of-flight mass spectrometry: concurrent acquisition of structural, molecular and elemental information. J. Anal. At. Spectrom. 2000, 15 (1), 19-25.

24. Lewis, C. L.; Moser, M. A.; Dale, D. E.; Hang, W.; Hassell, C.; King, F. L.; Majidi, V., Time-Gated Pulsed Glow Discharge: Real-Time Chemical Speciation at the Elemental, Structural, and Molecular Level for Gas Chromatography Time-of-Flight Mass Spectrometry. Anal. Chem. 2003, 75 (9), 1983-1996.

25. Fliegel, D.; Fuhrer, K.; Gonin, M.; Günther, D., Evaluation of a pulsed glow discharge time-of-flight mass spectrometer as a detector for gas chromatography and the influence of the glow discharge source parameters on the information volume in chemical speciation analysis. Analytical and bioanalytical chemistry 2006, 386 (1), 169-179.

26. Lewis, C. L.; Oxley, E. S.; Pan, C. K.; Steiner, R. E.; King, F. L., Determination of 40Ca+ in the Presence of 40Art: An Illustration of the Utility of Time-Gated Detection in Pulsed Glow Discharge Mass Spectrometry. Anal. Chem. 1999, 71 (1), 230-234.

27. Lobo, L.; Pisonero, J.; Bordel, N.; Pereiro, R.; Tempez, A.; Chapon, P.; Michler, J.; Hohl, M.; Sanz-Medel, A., A comparison of non-pulsed radiofrequency and pulsed radiofrequency glow discharge orthogonal time-of-flight mass spectrometry for analytical purposes. J. Anal. At. Spectrom. 2009, 24 (10), 1373-1381.

28. Steiner, R. E.; Lewis, C. L.; King, F. L., Time-of-Flight Mass Spectrometry with a Pulsed Glow Discharge lonization Source. Anal. Chem. 1997, 69 (9), 1715-21.

29. Hang, W. E. I.; Baker, C.; W. Smith, B.; Winefordner, J. D.; Harrison, W. W., Microsecond-pulsed Glow Discharge Time-of-flight Mass Spectrometry: Analytical Advantages. J. Anal. At. Spectrom. 1997, 12 (2), 143-149.

30. Myers, D. P.; Heintz, M. J.; Mahoney, P. P.; Li, G.; Hieftje, G. M., Characterization of a Radio-Frequency Glow Discharge/Time-of-Flight Mass Spectrometer. Appl. Spectrosc. 1994, 48 (11), 1337-1346. 
31. Cotter, R. J., Time-of-flight Mass Spectrometry: Instrumentation and Applications in Biological Research. American Chemical Society: 1997.

32. Duckworth, D. C.; Marcus, R. K., Radio frequency powered glow discharge atomization/ionization source for solids mass spectrometry. Anal. Chem. 1989, 61 (17), 18791886.

33. Duckworth, D. C.; Donohue, D. L.; Smith, D. H.; Lewis, T. A.; Marcus, R. K., Design and characterization of a radio-frequency-powered glow discharge source for double-focusing mass spectrometers. Anal. Chem. 1993, 65 (18), 2478-2484.

34. Marcus, R. K.; Broekaert, J. A., Glow discharge plasmas in analytical spectroscopy. John Wiley \& Sons: 2003.

35. Jakubowski, N.; Stuewer, D.; Toelg, G., Microchemical determination of platinum and iridium by glow discharge mass spectrometry. Spectrochimica Acta Part B: Atomic Spectroscopy 1991, 46 (2), 155-163.

36. Barshick, C. M.; Duckworth, D. C.; Smith, D. H., Analysis of solution residues by glow discharge mass spectrometry. J. Am. Soc. Mass. Spectrom. 1993, 4 (1), 47-53.

37. Scott, R. P. W.; Scott, C. G.; Munroe, M.; Hess, J., Interface for on-line liquid chromatography—mass spectroscopy analysis. J. Chromatogr. A 1974, 99, 395-405.

38. Games, D. E.; Martínez, F., Evaluation of the moving belt as an interface for the highperformance liquid chromatographic-mass spectrometric analysis of the flavonoid aglycones. $\mathrm{J}$. Chromatogr. A 1989, 474 (2), 372-380.

39. Willoughby, R. C.; Browner, R. F., Monodisperse aerosol generation interface for combining liquid chromatography with mass spectroscopy. Anal. Chem. 1984, 56 (14), 26262631.

40. Creaser, C. S.; Stygall, J. W., Particle beam liquid chromatography-mass spectrometry: instrumentation and applications. A review. The Analyst 1993, 118 (12), 1467-1480. 
41. Brewer, T. M.; Castro, J.; Marcus, R. K., Particle beam sample introduction into glow discharge plasmas for speciation analysis. Spectrochimica Acta Part B: Atomic Spectroscopy 2006, 61 (2), 134-149.

42. Schwartz, A. J.; Williams, K. L.; Hieftje, G. M.; Shelley, J. T., Atmospheric-pressure solution-cathode glow discharge: A versatile ion source for atomic and molecular mass spectrometry. Anal. Chim. Acta 2017, 950, 119-128.

43. Marcus, R. K.; Quarles, C. D.; Barinaga, C. J.; Carado, A. J.; Koppenaal, D. W., Liquid Sampling-Atmospheric Pressure Glow Discharge Ionization Source for Elemental Mass Spectrometry. Anal. Chem. 2011, 83 (7), 2425-2429.

44. Cserfalvi, T.; Mezei, P.; Apai, P., Emission studies on a glow discharge in atmospheric pressure air using water as a cathode. J. Phys. D: Appl. Phys. 1993, 26 (12), 2184.

45. Cserfalvi, T.; Mezei, P., Operating mechanism of the electrolyte cathode atmospheric glow discharge. Fresenius' Journal of Analytical Chemistry 1996, 355 (7), 813-819.

46. Webb, M. R.; Chan, G. C. Y.; Andrade, F. J.; Gamez, G.; Hieftje, G. M., Spectroscopic characterization of ion and electron populations in a solution-cathode glow discharge. J. Anal. At. Spectrom. 2006, 21 (5), 525-530.

47. He, Q.; Zhu, Z.; Hu, S., Flowing and Nonflowing Liquid Electrode Discharge Microplasma for Metal Ion Detection by Optical Emission Spectrometry. Applied Spectroscopy Reviews 2014, 49 (3), 249-269.

48. Marcus, R. K.; Manard, B. T.; Quarles, C. D., Liquid sampling-atmospheric pressure glow discharge (LS-APGD) microplasmas for diverse spectrochemical analysis applications. J. Anal. At. Spectrom. 2017, 32 (4), 704-716.

49. Zhang, L. X.; Marcus, R. K., Mass spectra of diverse organic species utilizing the liquid sampling-atmospheric pressure glow discharge (LS-APGD) microplasma ionization source. J. Anal. At. Spectrom. 2016, 31 (1), 145-151. 
50. Schwartz, A. J.; Shelley, J. T.; Walton, C. L.; Williams, K. L.; Hieftje, G. M., Atmosphericpressure ionization and fragmentation of peptides by solution-cathode glow discharge. Chemical Science 2016, 7 (10), 6440-6449.

51. Hoegg, E. D.; Barinaga, C. J.; Hager, G. J.; Hart, G. L.; Koppenaal, D. W.; Marcus, R. K., Isotope ratio characteristics and sensitivity for uranium determinations using a liquid samplingatmospheric pressure glow discharge ion source coupled to an Orbitrap mass analyzer. J. Anal. At. Spectrom. 2016, 31 (12), 2355-2362.

52. Aebersold, R.; Mann, M., Mass spectrometry-based proteomics. Nature 2003, 422 (6928), 198-207.

53. Yan, X.; Yang, L.; Wang, Q., Detection and quantification of proteins and cells by use of elemental mass spectrometry: progress and challenges. Analytical and bioanalytical chemistry 2013, 405 (17), 5663-70.

54. Wang, M.; Feng, W.; Lu, W.; Li, B.; Wang, B.; Zhu, M.; Wang, Y.; Yuan, H.; Zhao, Y.; Chai, Z., Quantitative analysis of proteins via sulfur determination by HPLC coupled to isotope dilution ICPMS with a hexapole collision cell. Anal. Chem. 2007, 79 (23), 9128-34.

55. Pereira Navaza, A.; Ruiz Encinar, J.; Sanz-Medel, A., Absolute and accurate quantification of protein phosphorylation by using an elemental phosphorus standard and element mass spectrometry. Angew. Chem. Int. Ed. Engl. 2007, 46 (4), 569-71.

56. Ballihaut, G.; Kilpatrick, L. E.; Kilpatrick, E. L.; Davis, W. C., Detection and characterization of selenoproteins by tandem mass spectrometry assisted by laser ablation inductively coupled plasma mass spectrometry: application to human plasma selenoproteins. J. Anal. At. Spectrom. 2011, 26 (2), 383-394.

57. Nuevo Ordonez, Y.; Montes-Bayon, M.; Blanco-Gonzalez, E.; Sanz-Medel, A., Quantitative analysis and simultaneous activity measurements of $\mathrm{Cu}, \mathrm{Zn}$-superoxide dismutase in red blood cells by HPLC-ICPMS. Anal. Chem. 2010, 82 (6), 2387-94. 
58. Liu, Z.; Li, X.; Xiao, G.; Chen, B.; He, M.; Hu, B., Application of inductively coupled plasma mass spectrometry in the quantitative analysis of biomolecules with exogenous tags: A review. TrAC, Trends Anal. Chem. 2017, 93, 78-101.

59. Liang, Y.; Yang, L.; Wang, Q., An ongoing path of element-labeling/tagging strategies toward quantitative bioanalysis using ICP-MS. Applied Spectroscopy Reviews 2016, 51 (2), 117-128.

60. Fliegel, D.; Waddell, R.; Majidi, V.; Guenther, D.; Lewis, C. L., Quantification of aromatic and halogenated hydrocarbons and alcohol mixtures at the elemental, structural, and parent molecular ion level. Anal. Chem. 2005, 77 (6), 1847-1852. 


\title{
Chapter 2
}

\section{Analysis of Cysteine Using Pulsed Glow Discharge Time-Of-}

\section{Flight Mass Spectrometry}

\begin{abstract}
Glow discharge mass spectrometry (GDMS) displays superior performance in direct analysis of solid samples. Application of conventional GD techniques to solution samples requires solvent removal before introduction to the ion source. RF pulsed glow discharge mass spectrometry has previously been applied to the analysis of amino acids. Analytes were sampled as dry residues directly deposited to the cathode. Previous study has established a characteristic fragment ion $(\mathrm{m} / \mathrm{z} 76)$ for cysteine, however, the sample prepared only produces a transient signal with low intensity, making it difficult for further studies. This continued study introduces a new sample preparation method. The efficiency of this method was compared with that of the previous method. Parametric studies were carried out to evaluate the influences of power, pressure and sampling distance. The cysteine fragmentation mechanism that produces the $m / z 76$ signal was explored.
\end{abstract}




\subsection{Introduction}

Glow discharge mass spectrometry is a powerful tool in the direct analysis of solid samples. The direct current-powered GD source readily analyzes conducting solids, whereas a radio-frequency powered discharge extends the application of GD sources to the direct analysis of semiconductor and nonconducting samples, such as glasses and ceramics. Recent application of GD techniques to the analysis of photovoltaic materials has successfully characterized the aluminum-doped $\mathrm{ZnO}$ films, ${ }^{1}$ boron implanted silicon, ${ }^{2-3}$ Perovskites solar cells, ${ }^{4}$ thin film tandem solar cells, ${ }^{5}$ as well as analyzed the impurities in silicon solar cells. ${ }^{6-7}$ Also, GDs are employed in the direct analysis of bulk and coated glasses ${ }^{8-9}$ and high depth resolution profiles of the layered materials can be obtained. Additional quantitative information such as the elemental concentration and layer thickness are also attainable with GDs using a multi-matrix calibration. ${ }^{10}$

Analytical GD sources can be equipped with continuous or modulated power supplies. Pulsed power operation in microsecond or millisecond pulses applies higher instantaneous power to the sputtering and ionization process, producing enhanced performance. In addition, a pulsed plasma places less thermal stress on the sample, making it particularly advantageous for the analysis of heat sensitive materials. Most importantly, pulsing the GD creates three time domains in the plasma that differs in predominant ionization process. Coupling a pulsed GD source to a time-gated acquisition system, such as a time-of-flight mass spectrometer would allow one to select the ionization environment to give rise to desired analyte signals. For example, using 
pulsed GD source as an ion detector for gas chromatography eluent has allowed the real-time speciation of a set of organic molecules at the atomic, structural and molecular level. ${ }^{11-12}$ This time resolved analysis also enables the identification and differentiation of polymer coatings of similar elemental composition according to the characteristic polyatomic ions formed specifically in the afterpeak regime. ${ }^{13-14}$ As is well known that the background species and sputtered analytes behave differently in a pulsed plasma, analyte signals could be obtained with minimum spectral interferences if the detection window is properly selected. This feature was fully illustrated in the studies of determining ${ }^{40} \mathrm{Ca}^{+}$and $\mathrm{Si}$ isotopes free from discharge species and polyatomic interferences in an Ar plasma. ${ }^{15-16}$

Analytes in solution state cannot be directly analyzed with the GD plasma because of the inefficiency of the desolvation process. Therefore, the analyte is commonly sampled as dry residues produced by evaporating a small aliquot of liquid sample in/on the host cathode. By this means, Jakuboswski and coworkers achieved the determination of subpicogram quantities of $\mathrm{Pt}$ and $\mathrm{Ir}$ elements in solution. ${ }^{17}$ However, this deposition method requires evaporation of solvent prior to sample introduction, thus is not applicable to sample solutions of a large volume. When LC eluents become the target sample, application of GD as a detector must come with a prior interface that removes the solvents efficiently. This kind of interface, such as a moving belt ${ }^{18}$ and particle beam interface, ${ }^{19}$ can be found in the studies of online coupling of LC to other low-pressure ion sources. Marcus ${ }^{20}$ provided a comprehensive review on the use of particle beam interface in the online coupling LC and GD. 
Nowadays, ambient GD sources ${ }^{21-22}$ have been invented using analyte solution as the cathode to perform analysis, but the efficiency of these source remains under evaluation.

Analysis of amino acids by GD techniques were reported in the literature. ${ }^{23-25}$ Amino acids including tyrosine, leucylglycine, ${ }^{23}$ tryptophan $^{24}$ as well as a number of selenoamino acids ${ }^{25}$ were analyzed in continuous GD plasmas employing diverse solution-sampling methods as previously discussed. In these analyses, molecular ion and structurally significant fragments were detected, and analyte discrimination was achieved based on the characteristic fragmentation patterns. Similarly, secondary-ion mass spectrometry ${ }^{26}$ was also used to perform a systematical investigation of the secondary-ion emission of amino acids. For all materials investigated, parent ions and abundant fragment ions were observed in both positive and negative spectra. Therefore, efforts were made to study the amino acid behavior in a pulsed radio frequency (RF) glow discharge plasma in this group. Previous studies ${ }^{27-28}$ have found that among all the amino acids tested, only cysteine produces a characteristic fragment ion within the current GDMS system. Here is a continued study to explore the feasibility of a new sample preparation method. A comparative study was performed to assess the overall efficiency of the new method in cysteine analysis. Parametric studies were conducted to evaluate the influences of power, pressure and sampling distance. A mechanism for the production of the cysteine fragment at $\mathrm{m} / \mathrm{z} 76$ was explored. 


\subsection{Experimental}

\subsubsection{Pulsed Radio Frequency Glow Discharge Time-of-Flight Mass}

\section{Spectrometry}

A six-way, high vacuum across (MDC Vacuum Products Co., Hayward, CA) serves as the ion source where GD ionization occurs. A $13.56 \mathrm{MHz}$ radio frequency (RF) generator (Model RF 10-S, RF Plasma Product Inc., Marlton, NJ) equipped with an automatic matching network (Model AM-10, RF Plasma Product Inc., Marlton, NJ) provides power to operate the GD. Operation of this power supply in a pulsed mode allows control of applied power, pulse width and duty cycle. The GD ionization chamber interfaces orthogonally with the linear time-of-flight mass spectrometer (R.M. Jordan Co., Grass valley $\mathrm{CA}$ ). This orientation allows pulsed injection of ions produced in the plasma into the flight tube by pulsing the repeller plate in the ion extraction region. A pulse generated with a variable delay relative to the initiation of the RF power pulse triggers the injection. Adjustment of the repeller pulse delay permits selection of ions formed in desired time regimes during the RF power pulse cycle. Detailed information about the TOF-MS system appears in Table 2.1. More information about the instrument configuration and data acquisition also appears in previous papers. ${ }^{27-28}$ 


\section{Table 2.1 TOF-MS operation parameters}

\section{Time-of-Flight}

Flight path length

$1 \mathrm{~m}$

\section{Ion lenses potential}

Skimmer

$-375 \mathrm{~V}$

Extractor

$0 \mathrm{~V}$

Accelerator

$-1535 \mathrm{~V}$

Deflector (X1)

$-1830 \mathrm{~V}$

Deflector $(\mathrm{Y} 1)$

$-1505 \mathrm{~V}$

Repeller

$+250 \mathrm{~V}$

Detector

$-1895 \mathrm{~V}$

\section{Vacuum conditions}

Intermediate stage $\quad 10^{-5}$ Torr

Flight tube $10^{-6}$ Torr

A Cu disk (with or without cysteine residue loaded) was placed on the tip of a direct insertion probe (DIP) and introduced into the GD chamber through a ball valve. It serves as the cathode. Cathodic sputtering was limited to the disk front surface specifically by means of surrounding the Cu disk with a nonconducting ceramic shield machined from MACOR (Accuratus, Washington, N.J.). Sampling distance refers to the distance from the front surface of the sample cathode to the ion exit orifice. It can be adjusted by moving the DIP position horizontally in the GD chamber and was within the range of $6-13 \mathrm{~mm}$ in this study. The GD power applied were studied in the range of 60 $100 \mathrm{~W}, 1-5 \mathrm{~ms}$ pulse and $10 \%-40 \%$ duty cycle. Argon (Ultra high purity, Matheson, 
Basking Ridge, $\mathrm{NJ}$ ) was used as the discharge gas and introduced to the GD chamber through a metering valve. Argon pressure in the GD chamber was monitored by a thermocouple pressure gauge (Varian, Lexington, MA) and controlled in the range 300 600 mTorr. Detailed GD operation conditions were provided with mass spectra shown.

\subsubsection{Cysteine Solution Preparation}

L-Cysteine (99\%, Acros Organics, Thermo Fisher Scientific, New Jersey, US) was dissolved in deionized water (18 $\mathrm{M} \Omega$, Barnstead NANOpure Infinity, Lake Balboa, CA) to prepare cysteine aqueous solutions. Based on previous study and quantification needs, a set of concentrations at $10,20,30,40,50 \mu \mathrm{g} / \mu \mathrm{L}$ were prepared. Solution of deuterated cysteine $\left(3,3^{-2} \mathrm{H}_{2}\right.$-Cysteine, $98 \%$, Cambridge Isotope Laboratories, Andover, MA) was prepare at a concentration of $20 \mu \mathrm{g} / \mu \mathrm{L}$ for comparative study.

\subsubsection{Modifications of Cu Disks for Residue Analysis}

Cysteine solutions cannot be directly analyzed by GDMS, therefore were introduced into the ion source as dry solution residues. Where and how the residue forms becomes a significant factor that influences the overall analytical performance. Cu disks of flat surfaces are not ideal devices to contain cysteine residues. Previous studies employed a $\mathrm{Cu}$ reservoir as residue container, whereas a new design was introduced here. So, 2 modifications of the regular $\mathrm{Cu}$ disks were compared with respect to their analytical performance. 
A copper rod (Alloy 110, McMaster-Carr Supply Company, Cleveland, OH) was machined to make two types of cylindrical Cu disks. The first type of disks is $5 \mathrm{~mm}$ diameter and $6 \mathrm{~mm}$ length, but the center has a small reservoir that is $3 \mathrm{~mm}$ diameter and $1 \mathrm{~mm}$ depth (Fig. 2.1 a). Cysteine solutions were added to the reservoir to prepare dry residues. The second design uses regular $\mathrm{Cu}$ disks with flat surfaces but adds a layer of supporting material on top of the surface. Cysteine solutions were added to the material and dried to make residues. The supporting material is expected to allow even distribution of the cysteine solution and better retain the solution residue for long-lasting signal. Materials tried on the $\mathrm{Cu}$ cathode include plastic paraffin film, polytetrafluoroethylene (PTFE), filter paper and membrane filter paper. Among all the materials tested, the use of hydrophilic membrane filter paper (EMD Millipore Corporation, Billerica, MA) is capable of producing a stable plasma and produces the best results, therefore the membrane was used for all experiments in this study. A round-cut membrane (15 $\mathrm{mm}$ diameter) was put on top of the disk. The membrane is larger than the disk surface, so extra portions were folded to the disk body to create a round surface similar to the disk surface. Finally, the extra portions were fixed to the disk body by parafilm. Fig. 2.1 b shows the 2nd modification of the copper disk (will be called "membrane" hereinafter). Theoretically, a Cu disk with a large surface would be preferred so that the residue produced would also have a large surface area, beneficial for broad sputtering. However, the size of the disk is limited by the sampling system. As mentioned in the instrumental description, the cathode disk is surrounded by a ceramic tube to limit sputtering to the front surface. So, the modified disk would need to fit in the ceramic tube ( $7 \mathrm{~mm}$ diameter). It was found that the original disks can have a 
maximal size of $4.5 \mathrm{~mm}$ diameter in order to fit in with membrane attached. Therefore, the second type of $\mathrm{Cu}$ disks are $4.5 \mathrm{~mm}$ diameter and $6 \mathrm{~mm}$ length. Note that, membrane layered disks are always prepared first, and then cysteine solutions are added.

(a) Cu reservoir

(b) Membrane layer on Cu disk
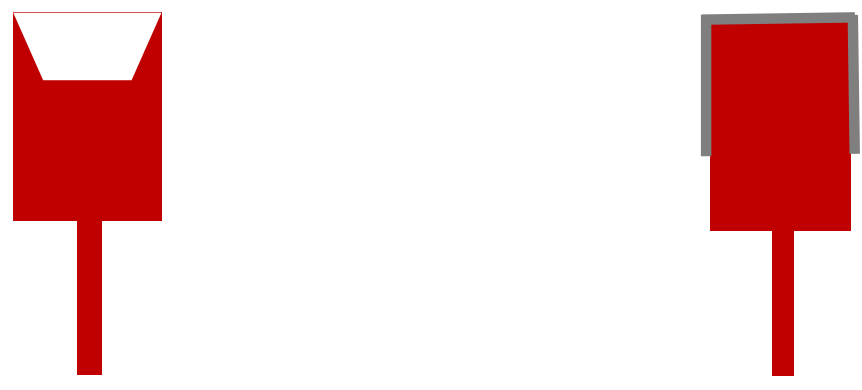

Figure 2.1 Schematic diagram of 2 modifications of Cu disks (a) Cu reservoir (b) Membrane layered Cu disk.

\subsubsection{Cysteine Residues Preparation}

Before use, $\mathrm{Cu}$ disks were baked in the oven at $110^{\circ} \mathrm{C}$ for 40 min to remove any residual water. The disks were then cooled down in a vacuum desiccator (Bel-art products, Pequannock, New Jersey). Cysteine solutions of desired concentrations and volumes were then deposited to the Cu reservoir (type1) or the membrane (type 2). The disks with sample loaded were then dried in the same vacuum desiccator to remove solvent and produce dry residues. For quantification attempt, aliquots of $4 \mu \mathrm{L}$ of 
the cysteine standard solutions were used to prepare residues. It was noticed that when adding aqueous solution to the membrane, the solution spreads quickly over the entire surface. So, each time only $2 \mu \mathrm{L}$ was added and dried for $30 \mathrm{~min}$ between deposits.

\subsection{Results and Discussion}

\subsubsection{Overview}

Pulsed glow discharge creates three temporal regimes that differ in plasma components and predominant ionization processes. Analysis of cysteine along the pulse cycle is expected to reveal different analytical information. However, according to Han's study ${ }^{28}$, the prepeak regime only provides background signals, whereas peaks relating to cysteine can be found in the plateau and afterpeak regimes. A primary peak can be observed at $m / z 76$. It arises in the plateau and significantly enhances in the afterpeak. Other than that, plateau shows background enhancement in the low mass range $(m / z 20-30)$, whereas afterpeak discloses signals in $m / z 50-60$. However, these signals, arising from intense fragmentation of the cysteine molecule, can't be employed for further analysis due to low intensity or overlap with discharge interferences. Although a fragment peak at $m / z 76$ was found in previous study, this signal displays low intensity and decreases quickly in analysis. These samples prepared in $\mathrm{Cu}$ reservoirs produced a cysteine fragment signal originating at 0.1-0.4 $\mathrm{V}$ and quickly decreasing to below $0.1 \mathrm{~V}$ in $10 \mathrm{~min}$. 


\subsubsection{Membrane Performance}

The residues prepared in Cu reservoir failed to produce a useful signal, therefore, a new approach to prepare residues was explored. A membrane placed on top of a $\mathrm{Cu}$ disk is intended to evenly adsorb the aqueous solution added and generate uniform residues that have a large surface area. Because it was unknown whether the membrane would influence the plasma property or produce interferences to the cysteine signal, experiments were undertaken to test the membrane behavior. A Cu reservoir was tested as well for comparison purpose. Fig. 2.2 are the afterpeak spectra collected at $2.1 \mathrm{~ms}$ for blank Cu reservoir and blank membrane.

It is encouraging to see that a discharge can be successfully established with a membrane-loaded $\mathrm{Cu}$ disk. The thin membrane material exhibits little influence to the Ar plasma. However, because of the membrane composition, a complex spectrum was obtained. Significantly enhanced background signals in the low mass range can be found, compared to that for a blank $\mathrm{Cu}$ reservoir, except that $\mathrm{Cu}$ signal is absent from the membrane spectrum because of the inhibited sputtering of the disk. In addition, a few peaks were found in $m / z 60-70$, arising from the membrane, but none interfere with the primary cysteine fragment at $m / z 76$. 
(a) Blank Cu reservoir

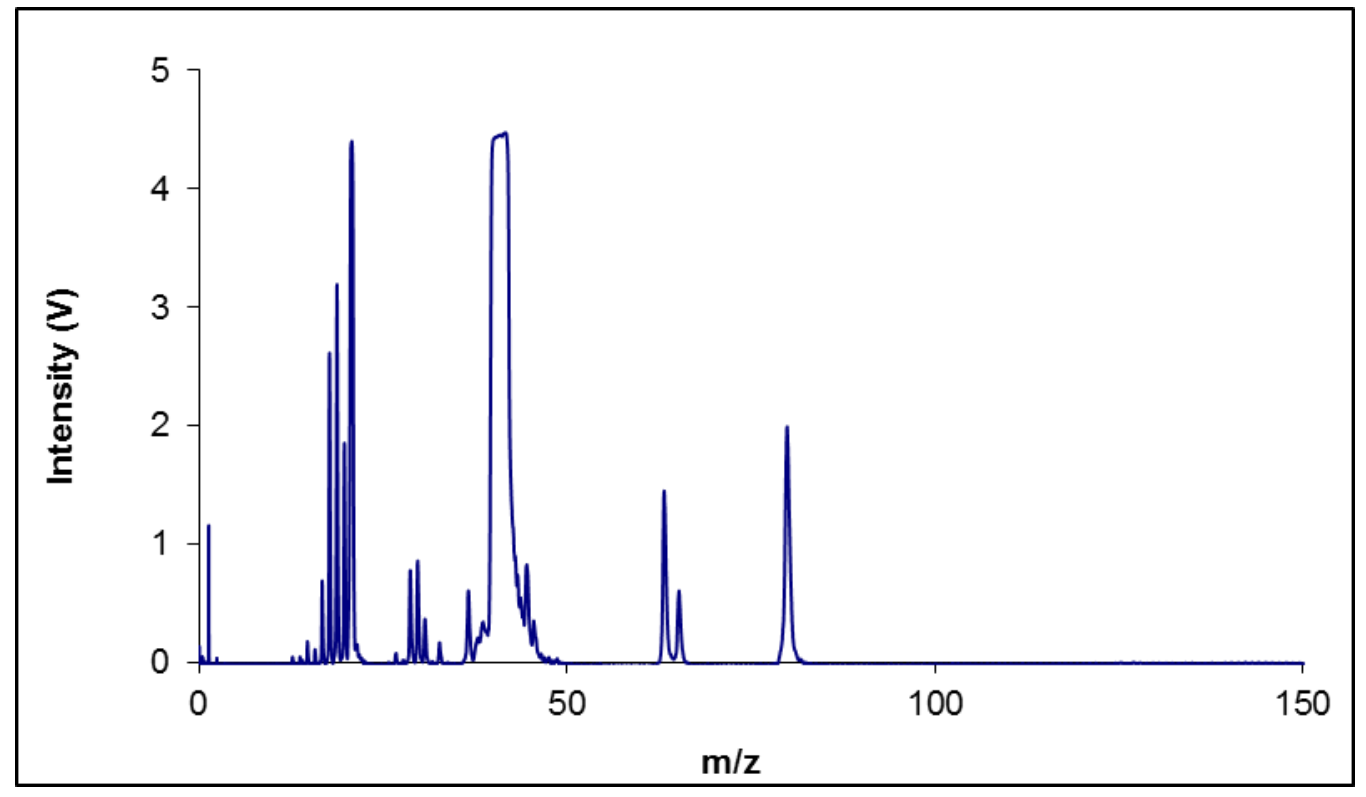

(b) Blank membrane

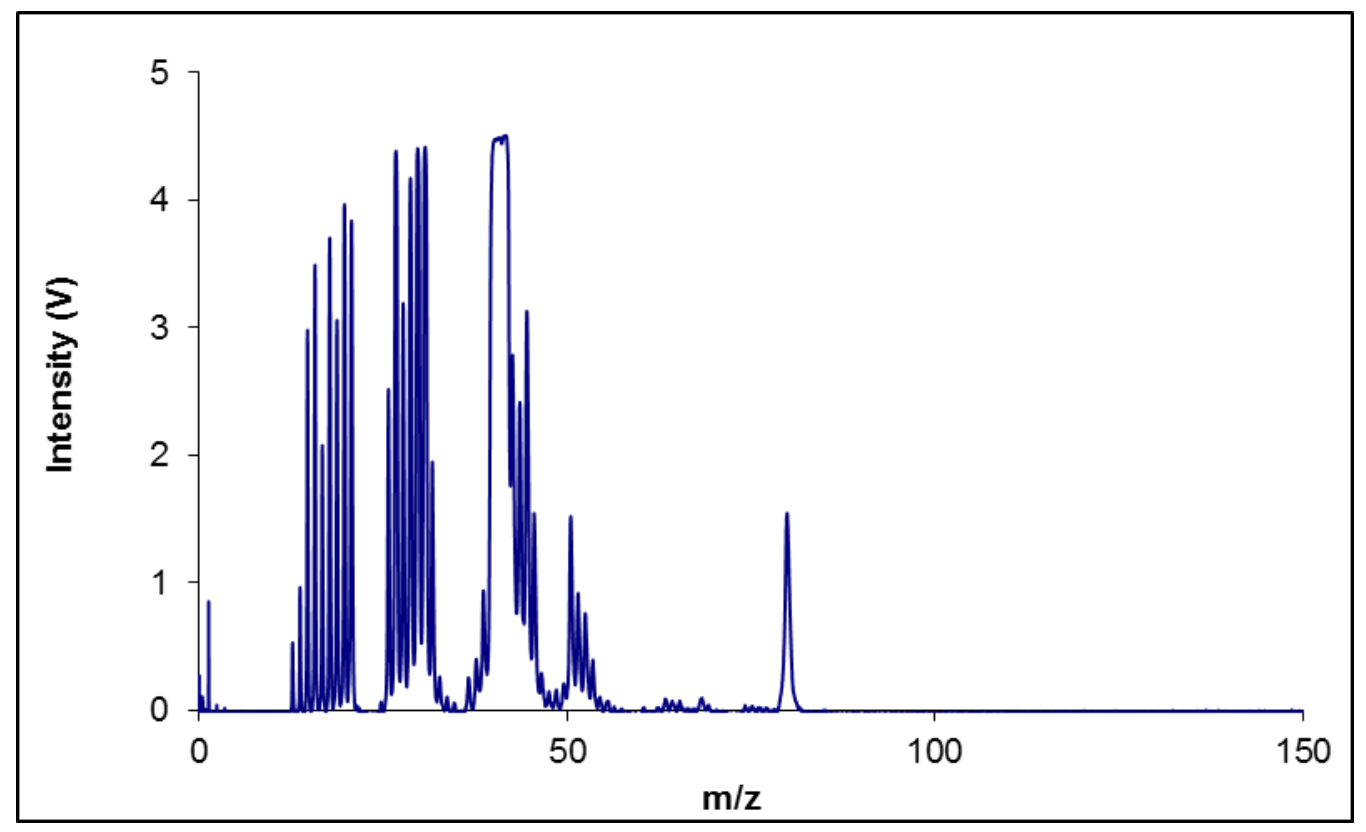

Figure 2.2 Mass spectra of (a) Blank Cu reservoir (b) Blank membrane during afterpeak (2.1 ms); GD operating conditions: 400 mTorr discharge pressure, $80 \mathrm{~W}$ RF power, $2 \mathrm{~ms}$ pulse width, $10 \%$ duty cycle, $7 \mathrm{~mm}$ sampling distance. 
Next, cysteine was tested in Cu reservoir and membrane. Residues containing same amounts of cysteine were prepared on each. Afterpeak spectra at $2.1 \mathrm{~ms}$ (Fig. 2.3) were collected to study the cysteine behavior on membrane. Identification of cysteine peaks was completed by comparing the blank and cysteine loaded membrane. With cysteine loaded, enhanced peaks at $m / z 32-35,46-48,64,65$ and new peaks at $\mathrm{m} / \mathrm{z}$ 57-61, 76, 78 were found. Among these peaks, peak at $\mathrm{m} / \mathrm{z} 76$ is most intense. And this signal is showing more intensity than that found in a Cu reservoir, although under current GD conditions, the latter is already showing improved performance and producing an intense cysteine signal. This test indicates that using the membrane to support cysteine residues enhances performance.

\section{(a) Cysteine in Cu reservoir}

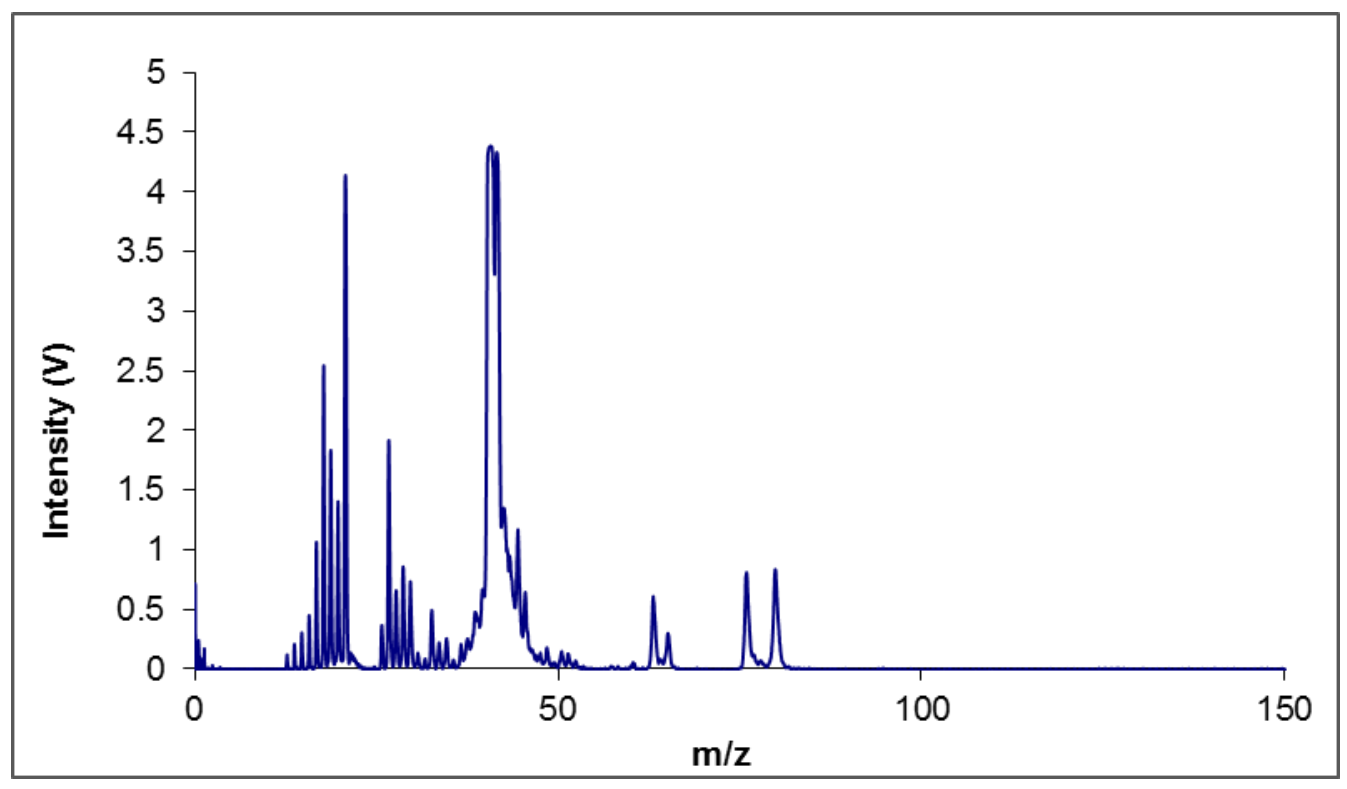




\section{(b) Cysteine on membrane}

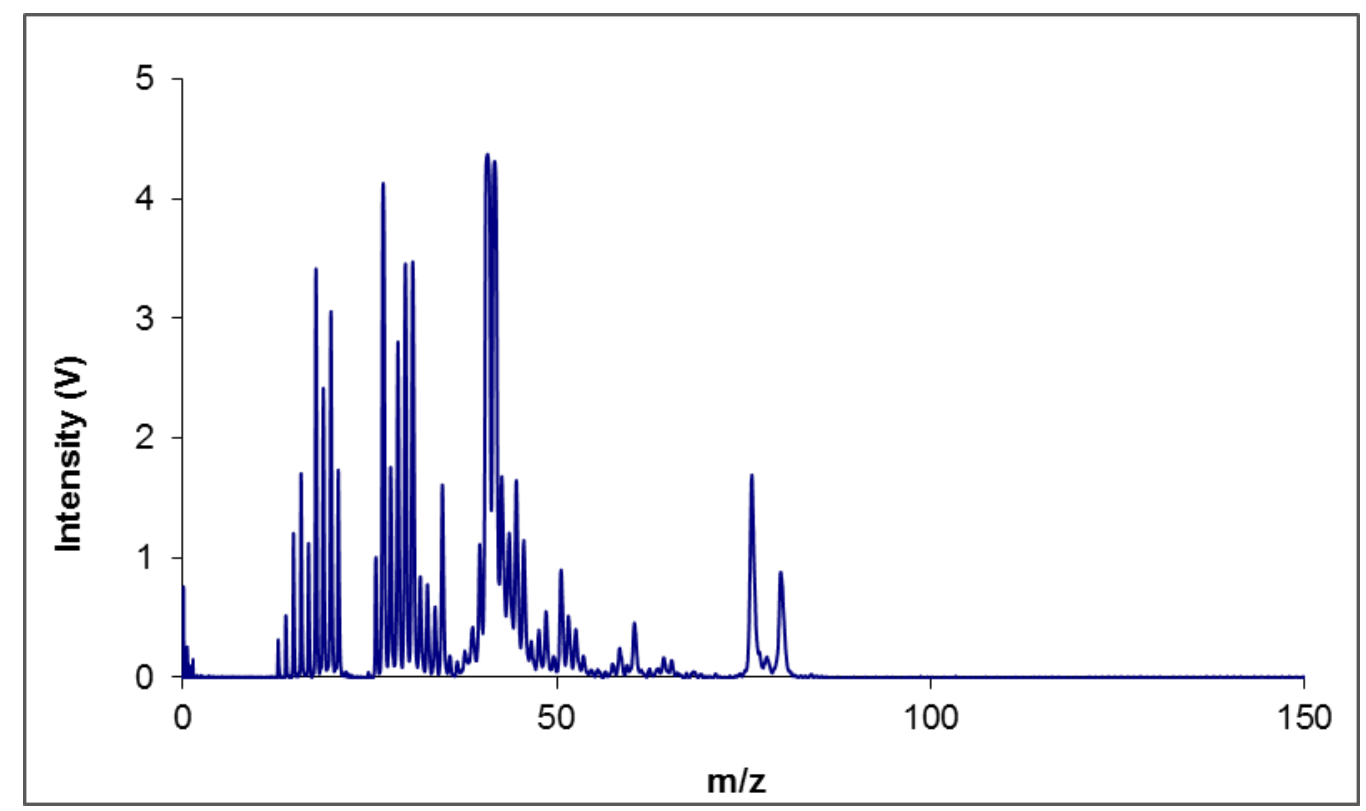

Figure 2.3 Mass spectra of cysteine on (a) Cu reservoir (b) Membrane during afterpeak (2.1 ms); GD operating conditions: 400 mTorr discharge pressure, 80 W RF power, 2 ms pulse width, $10 \%$ duty cycle, $7 \mathrm{~mm}$ sampling distance.

\subsubsection{Comparative Study: Cu Reservoir vs Membrane}

Experiments were undertaken to examine the cysteine fragment signal $(\mathrm{m} / \mathrm{z} 76)$ in terms of intensity and stability for both devices. Although the membrane produces a higher signal, further examination is needed to determine the performance of membrane filter. Residues of equal amounts of cysteine were prepared on a $\mathrm{Cu}$ reservoir and a membrane. Four replicates were prepared for each device. Cysteine fragment signal $(\mathrm{m} / \mathrm{z} 76)$ was collected in the afterpeak $(2.1 \mathrm{~ms})$ and plotted against the analysis time to gain an overview of the signal intensity for the entire analysis (Fig. 2.4). 
With the $\mathrm{Cu}$ reservoir, cysteine residue produces an intense signal initially. The signal intensity is about $1.5 \mathrm{~V}$. But it drops quickly in the first $10 \mathrm{~min}$. This trend implies that the sputtering process is consuming a lot of sample residues from the Cu reservoir. Less and less cysteine residue remains in the reservior and thus a decreasing signal is observed. At the end of the test (16 min), the residue left is only producing a signal about $15 \%$ of the original intensity.

The membrane-supported residue behaves quite differently. Initial signal observed is about the same level as that was produced with the Cu reservoir. But this signal is found to preserve most of its intensity by the end of the test. It goes through a gentle increase in the first $5 \mathrm{~min}$ and decreases slowly afterwards. After $15 \mathrm{~min}$ analysis, the residue is still able to produce a signal of $80 \%$ of the original value. This long-lasting signal is attributed to the merits of applying a membrane as residue host. Because of the membrane's hydrophilic nature, cysteine solution is believed to evenly spread over the surface, producing residues of a large surface area. In addition, the thin and porous material tends to retain the residues inside the pores but not to a depth that would affect sputtering. A comparable cysteine signal found at beginning confirms the efficient sputtering of residues kept in pores. Furthermore, the intense signal found after 15 min indicates the fact that hosting cysteine residues in membrane results in a less significant sample loss during sputtering. Clearly from the comparison of the two designs, one can see that membrane demonstrates superior performance to $\mathrm{Cu}$ reservoir. 


\section{(a) Cvsteine in Cu Reservoir}

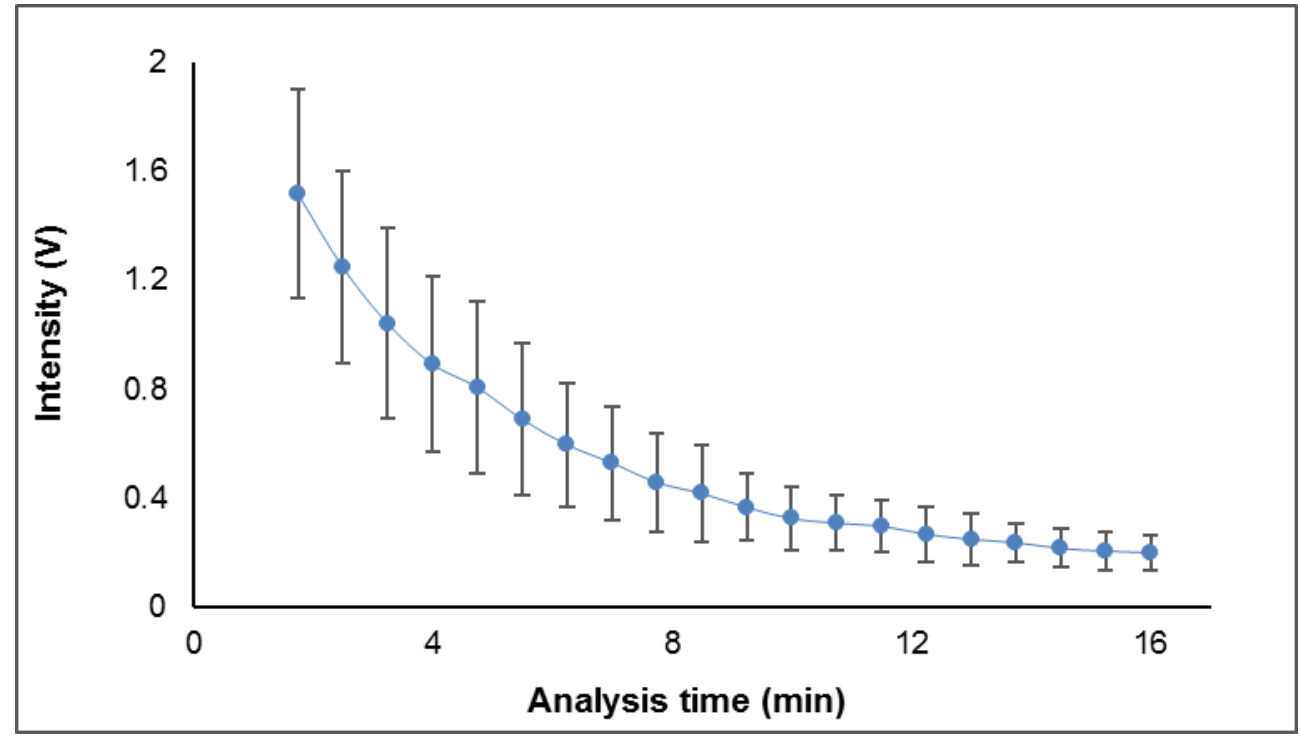

(b) Cysteine on membrane

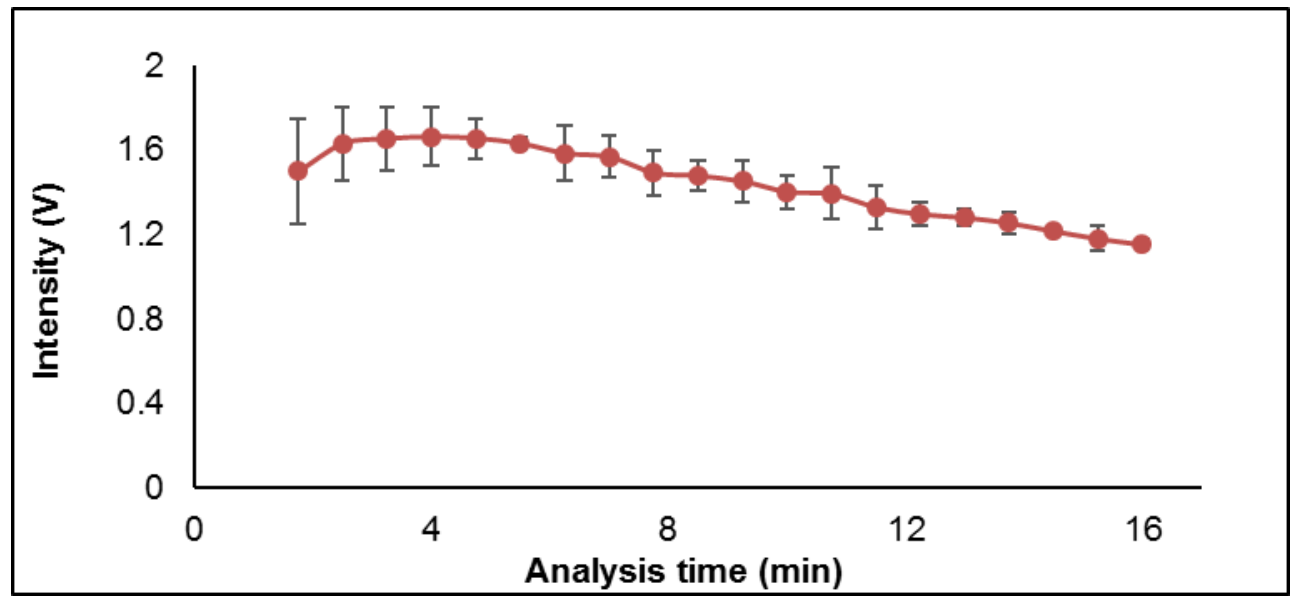

Figure 2.4 Cysteine fragment ion intensities for residues prepared on (a) Cu reservoir (b) Membrane; GD operating conditions: 400 mTorr discharge pressure, $80 \mathrm{~W}$ RF power, 2 ms pulse width, 10\% duty cycle, $7 \mathrm{~mm}$ sampling distance. Spectra collected in afterpeak regime $(2.1 \mathrm{~ms})$. Each data point represents averaged intensity from 4 replicates and error bars represent one standard deviation from the mean value. 


\subsubsection{Evaluation of GD Operating Parameters}

Because of the long-lasting cysteine signal obtained with membrane, one can evaluate the influences of the GD operating parameters and optimize those parameters. In the following experiments, five factors including discharge gas pressure, sampling distance, GD power, pulse width and duty cycle were evaluated separately for their influences on the cysteine signal. Results were displayed in Fig. 2.5 .

Pressure of the discharge gas, representing the density of argon atoms in the plasma, determines the mean free path and collision frequency in the gas phase. At low pressure, a small number of argon atoms is supplied to the plasma, leading to poor sputtering and ionization efficiencies. Therefore, pressure increase from 300 to 500 mTorr translates to an input of a higher number of sputtering and ionizing agents, and thus produces an increased signal (Fig. 2.5 a). Further increase of pressure provides a larger population of charge carriers (more conductivity), which in turn results in a lower voltage plasma environment for the same current. The charge carriers in this environment would end with less kinetic energy, lowering the sputtering efficiency. In addition, redeposition of the sputtered material and collisional deexcitation of the metastable argon atoms is found to increase with pressure, leading to loss of analyte and ionizing species respectively.

Sampling distance is controlled by the position of the DIP in the GD chamber. Moving the DIP closer to the anode reduces the sampling distance and increasing distance can be achieved by moving DIP away from the anode. Cysteine residue was 
sampled in the range of $6-13 \mathrm{~mm} .6 \mathrm{~mm}$ is chosen to start because no spectrum can be obtained less than $6 \mathrm{~mm}$. And this test ends at $13 \mathrm{~mm}$ where an obvious decrease of signal was seen. It was found that the optimal sampling distance for cysteine is $7-8 \mathrm{~mm}$ (Fig. 2.5 b). This is consistent with the previous findings ${ }^{29}$ that it is where the maximal metastable argon population resides.

Because pulsed power is supplied to the plasma, operating conditions including the power, pulse width and duty cycle were evaluated separately. Increase of power enhances sputtering and ionization, so an increased signal was observed (Fig. 2.5 c). Evaluation of pulse width (Fig. $2.5 \mathrm{~d}$ ) and duty cycle (Fig. 2. 5 e) shows that a high signal is readily obtained at a small pulse width $(2 \mathrm{~ms})$ and low duty cycle $(10 \%)$. This is because a short pulse with a small duty cycle would allow the application of a high instantaneous power for a short period of time. It not only enhances the sputtering and ionization process, but also allow the analyte to have more time to cool or recover between sputtering periods. However, a pulse of $1 \mathrm{~ms}$ width would be too short to produce an ideal signal, because there is not sufficient time for the plasma to reach a quasi-steady state.

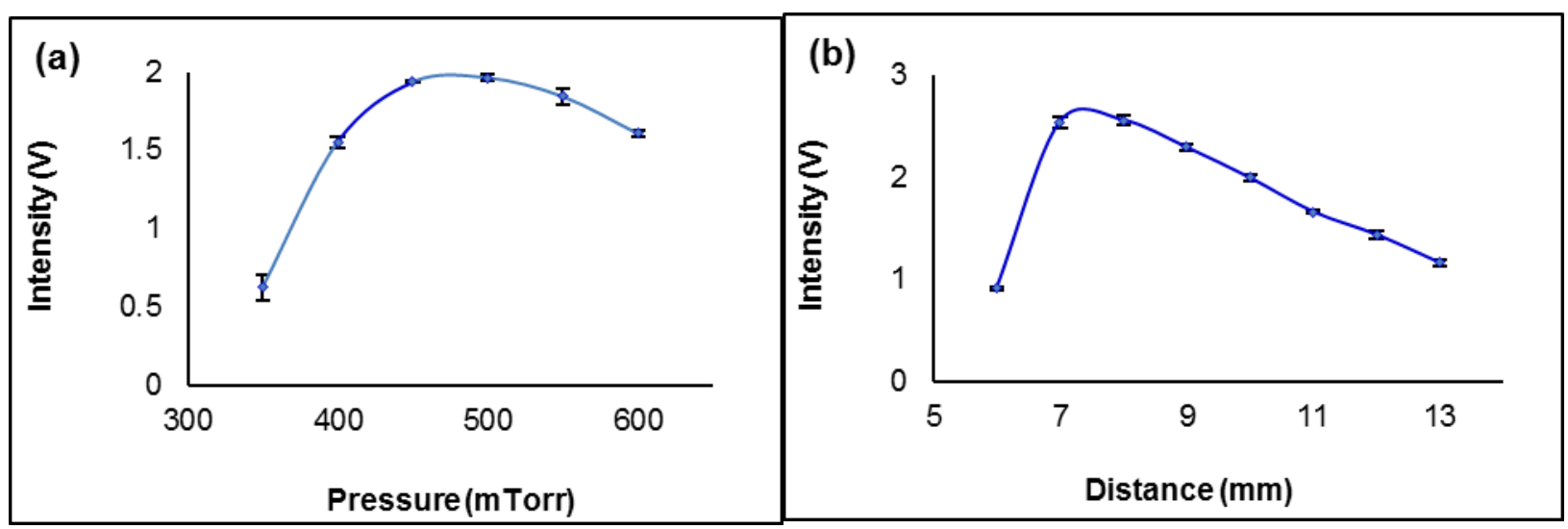



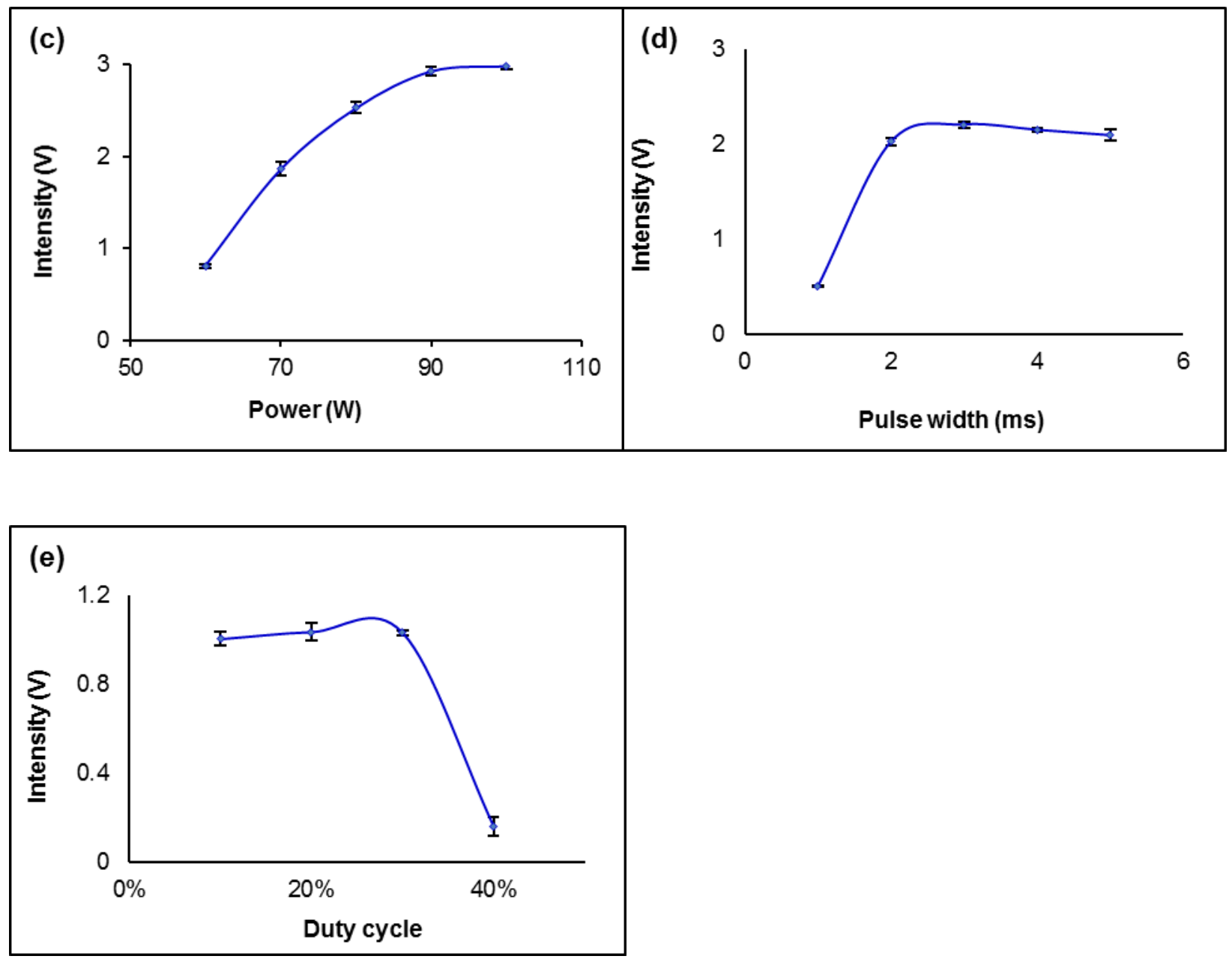

Figure 2.5 Evaluation of GD operating conditions influences on cysteine fragment signal (a) Discharge pressure (b) Sampling distance (c) RF power (d) Pulse width (e) Duty cycle; GD operating parameters employed for (a,b,c,e): 70 W RF power, 2 ms pulse width, $10 \%$ duty cycle, $400 \mathrm{mTorr}$ discharge pressure, $7 \mathrm{~mm}$ sampling distance except the one being varied for evaluation. For (d), $25 \%$ duty cycle, other parameters are the same. Each data point is the averaged value obtained from 3 measurements and error bars represent one standard deviation from the mean value. 


\subsubsection{Quantification and Reproducibility}

Under optimized conditions, quantification using the membrane was attempted. Cysteine residues prepared from solutions at different concentrations were analyzed. For each concentration, 3 replicates were prepared. Cysteine fragment signal obtained for each concentration was plotted against the concentration in Fig. 2.6. An increasing cysteine signal was observed when increasing the concentration from 10 to $50 \mu \mathrm{g} / \mu \mathrm{L}$. It was expected because the cysteine solution at high concentration deposits more cysteine in the residue, yielding a higher ion signal. However, when comparing the signals for the same concentration, a large deviation could be observed, especially for high concentrations. Samples prepared from $10 \mu \mathrm{g} / \mu \mathrm{L}$ solution produce relatively stable signals, while $20,30,40,50 \mu \mathrm{g} / \mu \mathrm{L}$ replicates are showing huge deviations that would make the method unreliable for quantitative analysis. This indicates that the membrane design is not highly reproducible. Reasons for low reproducibility might be associated with the manual fabrication of the membrane-loaded disks. A method to reproducibly attach membrane and create identical surfaces is needed to improve this aspect of performance. 


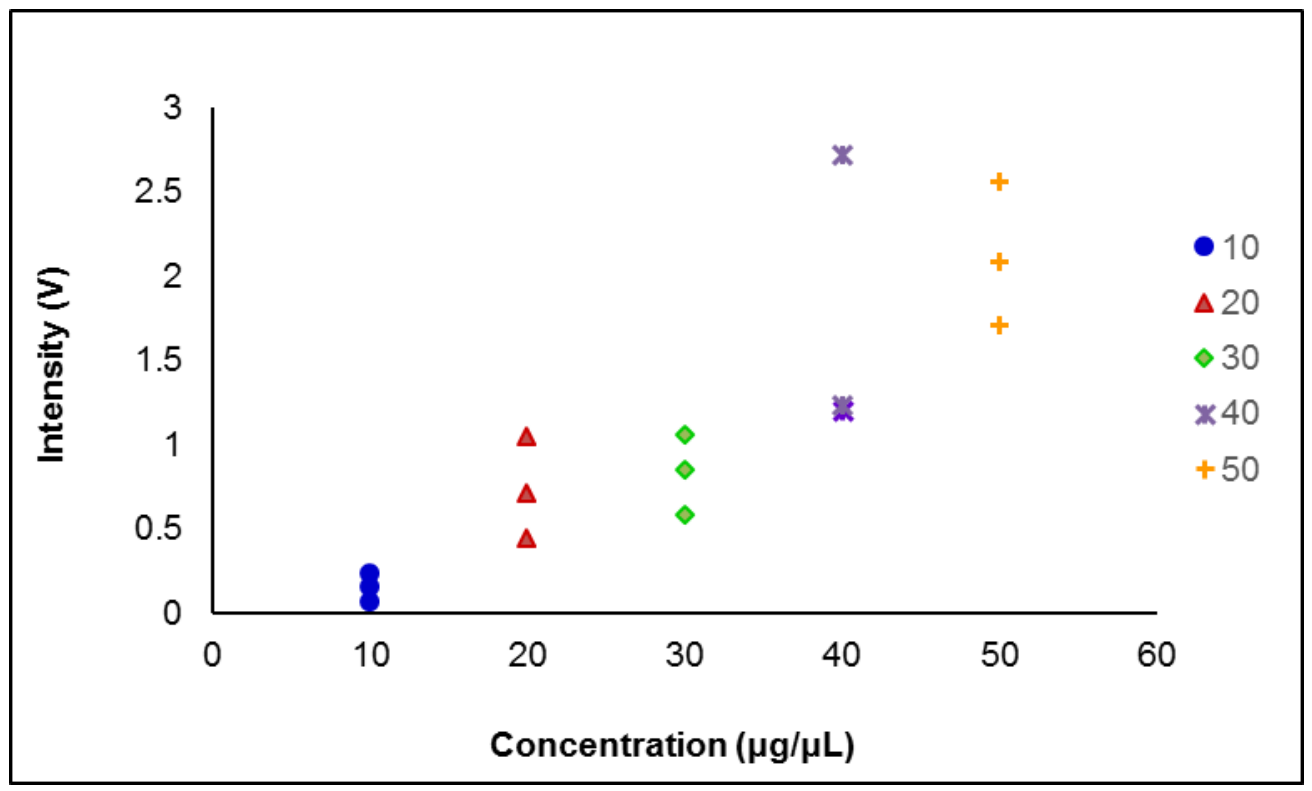

Figure 2.6 Cysteine fragment ion intensities for samples prepared from different concentrations; GD operating conditions: 400 mTorr discharge pressure, $80 \mathrm{~W}$ RF power, $2 \mathrm{~ms}$ pulse width, $10 \%$ duty cycle, $7 \mathrm{~mm}$ sampling distance. Spectra collected in afterpeak regime $(2.1 \mathrm{~ms})$. Each data point represents the averaged signal from 2-5 $\min$.

\subsubsection{Comparison with deuterated cysteine $\left(3,3-{ }^{2} \mathrm{H}_{2}\right.$-Cysteine)}

Previous studies attribute the presence of the fragment peak at $\mathrm{m} / \mathrm{z} 76$ to loss of carboxylic acid group (-COOH) from the cysteine molecule. ${ }^{26-28}$ To further look into the fragmentation process, deuterated cysteine was introduced in the analysis for comparison. Deuterated cysteine has 2 deuteriums replacing the hydrogens on the side chain carbon (Fig. 2.7). It has a molecular mass of $123 \mathrm{~g} / \mathrm{mol}$, relative to $121 \mathrm{~g} / \mathrm{mol}$ for cysteine. Because of the exact same structure, it is assumed that deuterated cysteine 
undergoes same sputtering and ionizing processes in the plasma. Therefore, the ion profile of deuterated cysteine should help to understand the behavior of cysteine in the plasma. If cysteine undergoes a loss of carboxylic acid group to produce a fragment at $\mathrm{m} / \mathrm{z} 76$, one would expect the deuterated cysteine to produce a primary fragment at $\mathrm{m} / \mathrm{z}$ 78 , since the 2 deuteriums remaining on side chain would add a mass of 2 to the original fragment.

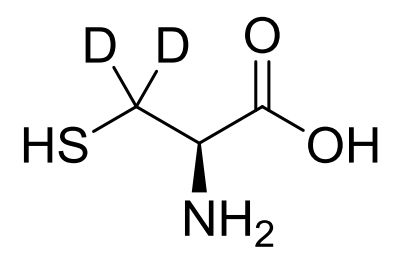

Figure 2.7 Structure of deuterated cysteine $\left(3,3-{ }^{2} \mathrm{H}_{2}\right.$-Cysteine) .

Ion profiles of cysteine and deuterated cysteine collected in the afterpeak regime are shown in Fig. 2.8. These two mass spectra do not show much difference. The fragment ion for deuterated cysteine is still found at $m / z 76$, instead of $m / z 78$. The absence of $\mathrm{m} / \mathrm{z} 78$ peak indicates that the deuteriums on the side chain are lost during fragmentation. Therefore, the loss of carboxylic acid theory is unable to explain this observation. In addition, methionine and glutathione, another two sulfur-containing biomolecules were tested as well using the same method. These two compounds both produce one primary signal at $m / z 76$ (data not shown). Due to the presence of this signal in the analysis of three sulfur-containing compounds, it is attributed to a 
characteristic ion containing sulfur. Based on its mass, a polyatomic ion $\mathrm{CS}_{2}{ }^{+}$is proposed. Detailed mechanism that is responsible for this ion is still under exploration.

(a) Cysteine

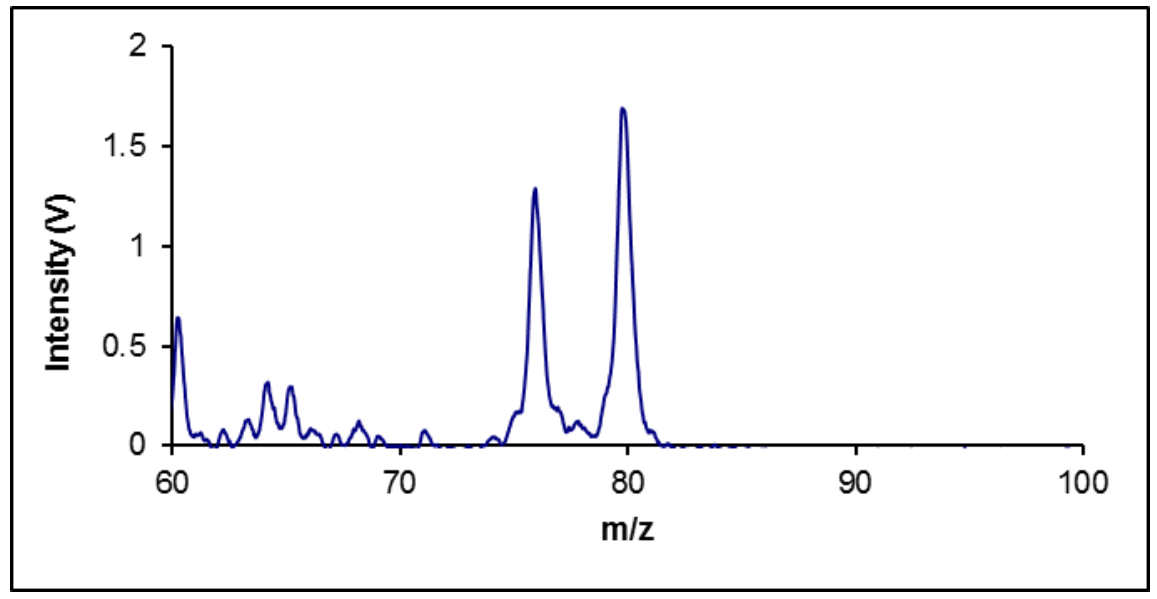

(b) D. Cysteine

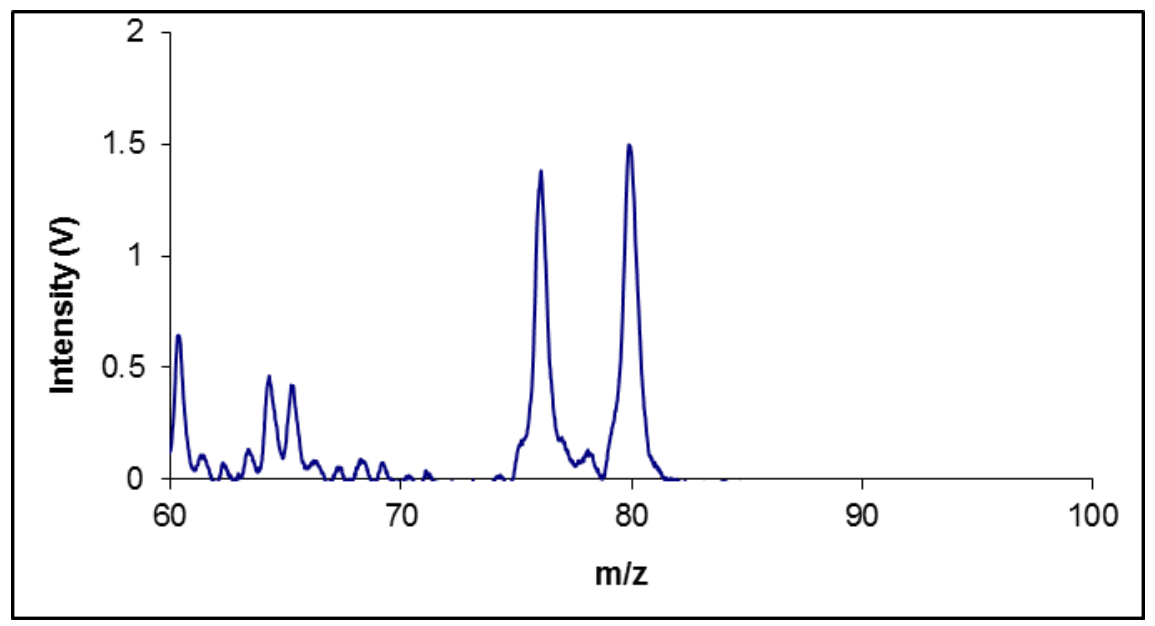

Figure 2.8 Mass spectra of (a) Cysteine (b) Deuterated cysteine during afterpeak (2.1 ms); GD operating conditions: 400 mTorr discharge pressure, 80 W RF power, 2 ms pulse width, $10 \%$ duty cycle, $7 \mathrm{~mm}$ sampling distance. 


\subsection{Conclusion}

The new sample preparation method that employs a membrane filter to accommodate cysteine residue has proved to be superior to the previous method in producing an intense and durable signal. Based on this improved signal, GD operating parameters including power, pressure, sampling distance, pulse width, duty cycle can be evaluated for their influence on the cysteine fragment signal. Under optimized conditions, cysteine solutions of different concentrations were tested in GDMS. The cysteine fragment ion signal obtained from replicates of the same concentration showed less reproducibility at high concentrations. Although an increasing signal could be obtained when increasing the concentration, the correlation between the fragment signal and cysteine concentration is not ideal. Fragmentation induced loss of carboxyl acid was attributed to be responsible for the cysteine fragment at $m / z 76$. But this theory fails to explain an identical fragment at $\mathrm{m} / \mathrm{z} 76$ for deuterated cysteine in a comparative study. The mechanism for production of this ion will be the topic of future investigations. 


\subsection{References}

1. Schmitt, S. W.; Gamez, G.; Sivakov, V.; Schubert, M.; Christiansen, S. H.; Michler, J., Chemical and optical characterisation of atomic layer deposition aluminium doped $\mathrm{ZnO}$ films for photovoltaics by glow discharge optical emission spectrometry. $\mathrm{J}$. Anal. At. Spectrom. 2011, 26 (4), 822-827.

2. Pisonero, J.; Lobo, L.; Bordel, N.; Tempez, A.; Bensaoula, A.; Badi, N.; SanzMedel, A., Quantitative depth profile analysis of boron implanted silicon by pulsed radiofrequency glow discharge time-of-flight mass spectrometry. Sol. Energy Mater. Sol. Cells 2010, 94 (8), 1352-1357.

3. Putyera, K.; Su, K.; Liu, C.; Hockett, R. S.; Wang, L., Quantitative Measurement of Boron and Phosphorus in Solar Grade Silicon Feedstocks by High Resolution FastFlow Glow-Discharge Mass Spectrometry. MRS Proceedings 2011, 1123.

4. Cojocaru, L.; Uchida, S.; Matsubara, D.; Matsumoto, H.; Ito, K.; Otsu, Y.; Chapon, P.; Nakazaki, J.; Kubo, T.; Segawa, H., Direct Confirmation of Distribution for $\mathrm{Cl}-$ in CH3NH3Pbl3-xClx Layer of Perovskite Solar Cells. Chem. Lett. 2016, 45 (8), 884-886.

5. Fernandez, B.; Lobo, L.; Reininghaus, N.; Pereiro, R.; Sanz-Medel, A., Characterization of thin film tandem solar cells by radiofrequency pulsed glow discharge - Time of flight mass spectrometry. Talanta 2017, 165, 289-296. 
6. Di Sabatino, M.; Dons, A. L.; Hinrichs, J.; Arnberg, L., Determination of relative sensitivity factors for trace element analysis of solar cell silicon by fast-flow glow discharge mass spectrometry. Spectrochimica Acta Part B: Atomic Spectroscopy 2011, 66 (2), 144-148.

7. Modanese, C.; Arnberg, L.; Di Sabatino, M., Analysis of impurities with inhomogeneous distribution in multicrystalline solar cell silicon by glow discharge mass spectrometry. Materials Science and Engineering: B 2014, 180, 27-32.

8. Pisonero, J.; Costa, J. M.; Pereiro, R.; Bordel, N.; Sanz-Medel, A., A radiofrequency glow-discharge-time-of-flight mass spectrometer for direct analysis of glasses. Analytical and bioanalytical chemistry 2004, 379 (4), 658-667.

9. Muniz, A. C.; Pisonero, J.; Lobo, L.; Gonzalez, C.; Bordel, N.; Pereiro, R.; Tempez, A.; Chapon, P.; Tuccitto, N.; Licciardello, A.; Sanz-Medel, A., Pulsed radiofrequency glow discharge time of flight mass spectrometer for the direct analysis of bulk and thin coated glasses. J. Anal. At. Spectrom. 2008, 23 (9), 1239-1246.

10. Bouza, M.; Pereiro, R.; Bordel, N.; Sanz-Medel, A.; Fernandez, B., Pulsed radiofrequency glow discharge time of flight mass spectrometry for coated glass analysis. J. Anal. At. Spectrom. 2015, 30 (5), 1108-1116.

11. Lewis, C. L.; Moser, M. A.; Dale, D. E.; Hang, W.; Hassell, C.; King, F. L.; Majidi, V., Time-Gated Pulsed Glow Discharge: Real-Time Chemical Speciation at the Elemental, Structural, and Molecular Level for Gas Chromatography Time-of-Flight Mass Spectrometry. Anal. Chem. 2003, 75 (9), 1983-1996. 
12. Fliegel, D.; Waddell, R.; Majidi, V.; Gunther, D.; Lewis, C. L., Quantification of aromatic and halogenated hydrocarbons and alcohol mixtures at the elemental, structural, and parent molecular ion level. Anal. Chem. 2005, 77 (6), 1847-52.

13. Lobo, L.; Tuccitto, N.; Bordel, N.; Pereiro, R.; Pisonero, J.; Licciardello, A.; Tempez, A.; Chapon, P.; Sanz-Medel, A., Polymer screening by radiofrequency glow discharge time-of-flight mass spectrometry. Analytical and bioanalytical chemistry 2010, 396 (8), 2863-2869.

14. Gonzalez de Vega, C.; Fernandez, B.; Bordel, N.; Pereiro, R.; Sanz-Medel, A., Challenging identifications of polymer coatings by radiofrequency pulsed glow discharge-time of flight mass spectrometry. J. Anal. At. Spectrom. 2013, 28 (7), 10541060.

15. Lewis, C. L.; Oxley, E. S.; Pan, C. K.; Steiner, R. E.; King, F. L., Determination of 40Ca+ in the Presence of 40Ar+: An Illustration of the Utility of Time-Gated Detection in Pulsed Glow Discharge Mass Spectrometry. Anal. Chem. 1999, 71 (1), 230-234.

16. Lobo, L.; Pisonero, J.; Bordel, N.; Pereiro, R.; Tempez, A.; Chapon, P.; Michler, J.; Hohl, M.; Sanz-Medel, A., A comparison of non-pulsed radiofrequency and pulsed radiofrequency glow discharge orthogonal time-of-flight mass spectrometry for analytical purposes. J. Anal. At. Spectrom. 2009, 24 (10), 1373-1381.

17. Jakubowski, N.; Stuewer, D.; Toelg, G., Microchemical determination of platinum and iridium by glow discharge mass spectrometry. Spectrochimica Acta Part B: Atomic Spectroscopy 1991, 46 (2), 155-163. 
18. Brackett, J. M.; Vickers, T. J., A new, glow discharge device with continuous flow sample introduction for trace and micro analysis. Spectrochimica Acta Part B: Atomic Spectroscopy 1983, 38 (7), 979-985.

19. Willoughby, R. C.; Browner, R. F., Monodisperse aerosol generation interface for combining liquid chromatography with mass spectroscopy. Anal. Chem. 1984, 56 (14), 2626-2631.

20. Brewer, T. M.; Castro, J.; Marcus, R. K., Particle beam sample introduction into glow discharge plasmas for speciation analysis. Spectrochimica Acta Part B: Atomic Spectroscopy 2006, 61 (2), 134-149.

21. Marcus, R. K.; Quarles, C. D.; Barinaga, C. J.; Carado, A. J.; Koppenaal, D. W., Liquid Sampling-Atmospheric Pressure Glow Discharge Ionization Source for Elemental Mass Spectrometry. Anal. Chem. 2011, 83 (7), 2425-2429.

22. Schwartz, A. J.; Williams, K. L.; Hieftje, G. M.; Shelley, J. T., Atmosphericpressure solution-cathode glow discharge: A versatile ion source for atomic and molecular mass spectrometry. Anal. Chim. Acta 2017, 950, 119-128.

23. Mason, R.; Milton, D., Glow discharge mass spectrometry of some organic compounds. Int. J. Mass Spectrom. Ion Processes 1989, 91 (2), 209-225.

24. Gibeau, T. E.; Marcus, R. K., Glow Discharge Ionization Source for Liquid Chromatography/Particle Beam Mass Spectrometry. Anal. Chem. 2000, 72 (16), 38333840. 
25. Gibeau, T. E.; Marcus, R. K., Separation and identification of organic and organometallic compounds by use of a liquid chromatography-particle beam-glow discharge mass spectrometry combination. J. Chromatogr. A 2001, $915(1-2), 117-28$.

26. Benninghoven, A.; Jaspers, D.; Sichtermann, W., Secondary-ion emission of amino acids. Applied physics 1976, 11 (1), 35-39.

27. Gu, G., Mass Spectrometric Approaches to Characterization of Chemical Species Containing Metallic lons. West Virginia University: 2012.

28. Wang, H. Ion Current Rectification in Nano/Micro-Fluidic Interfaces and Pulsed Glow Discharge Time-of-Flight Mass Spectrometric Chemical Speciation. West Virginia University, 2014.

29. DeJesus, M. R.; Gu, G.; King, F. L.; Barnes, J. H.; Lewis, C. L., lon formation in millisecond pulsed glow discharge plasmas. J. Anal. At. Spectrom. 2011, 26 (11), 22062215. 


\title{
Chapter 3
}

\section{Extending the Applicability of Pulsed Glow Discharge Mass}

\author{
Spectrometry to GHK-Cu Determination
}

\section{Abstract}

Elemental mass spectrometry is a useful alternative for protein/peptide quantification if naturally occurring or chemically tagged elements enable the quantification to be completed via the measurement of one specific element/isotope. ${ }^{1}$ Glow discharge mass spectrometry (GDMS) is a powerful technique for trace elemental analysis of solid samples and its extension to peptide solutions is explored here. The present work investigates the analytical potential of a pulsed RF glow discharge time-offlight mass spectrometry (GD-TOF MS) for the quantitative determination of glycyl-Lhistidyl-L-lysine copper (GHK-Cu) complex. Peptide solutions can not be directly analyzed by low-pressure glow discharge (GD) sources, so they were introduced to GD plasma as solution residues. Sample preparation involves depositing a microliter volume of peptide solution on a silver cathode, followed by vacuum drying to evaporate the solvent prior to GD analysis. Pulsed GD in combination with TOF MS permits selection of ionization mechanisms in different temporal regimes along the pulse, so peptide behavior along the pulse was first investigated to determine the optimum 
window for analytical work. In particular, the ${ }^{63} \mathrm{Cu}^{+}$ion was tracked and showed the highest intensity in the afterpeak regime. Sampling distance and GD operating conditions were evaluated for their influences on the ${ }^{63} \mathrm{Cu}^{+}$ion signal and optimized conditions were identified that yielded the highest sensitivity. Finally, the quantitative ability of this approach was explored by analyzing a set of GHK-Cu standards, and correlating the ${ }^{63} \mathrm{Cu}^{+}$ion intensity with the peptide solution concentration. The obtained calibration curve showed good linearity, demonstrating the potential of GDMS in peptide quantification through elemental detection.

\subsection{Introduction}

Pulsed radio frequency (RF) glow discharge mass spectrometry (GDMS) is attracting great interest for its superior performance in direct analysis of solid samples. ${ }^{2}$ A glow discharge (GD) generated with RF power has no requirements for sample conductivity, extending its application to a wide variety of materials, including glasses, ${ }^{3}$ polymers ${ }^{4-5}$ and solar cells. ${ }^{6-7}$ Moreover, pulsing the GD further enhances the analytical capability by supplying higher instantaneous power, without inducing overheating problems that usually accompanies continuous GDs operated under high power. The most attractive feature, as well as the reason behind the extensive applications of pulsed GDs, lies in the three temporal regimes (prepeak, plateau, afterpeak) established along a pulse cycle. Sample analysis conducted in these regimes benefits from different ionization mechanisms (electron impact, charge transfer, Penning ionization), so analyte information at several different levels can be obtained. ${ }^{8-10}$ Coupling a pulsed GD with time-of-flight mass spectrometry (TOF MS) affords the 
possibility of temporal separation of the ionization mechanisms. With the use of detection gates at different time windows along the pulse, time-resolved studies are attainable to select the best sensitivity for analyte detection as well as to minimize spectral interferences. ${ }^{11}$

The application of GD ion sources to solution analysis can be challenging. Solvent-related problems, like incomplete desolvation or quenching of plasma energetics are the major obstacles encountered in GD analysis of solution samples. ${ }^{12} \mathrm{~A}$ viable approach is the use of solution residues with GD sources for analytical work. ${ }^{13-15}$ Generally, solution residues are prepared by depositing an aliquot of analyte-containing solution in or on a conducting host cathode and subsequently removing the solvent by evaporation. The cathode material is required to have high sputtering rate and sufficient purity to allow successful analysis of residues. For solutions of a large volume, such as liquid chromatography (LC) eluents, interfaces like a moving belt and particle beam interface are employed to deliver solvent-free analyte particles to GD sources. ${ }^{16-17}$ More recently, direct solution analysis has become possible with GD source operating at ambient pressure..$^{18-19}$

In this work, we explore the application of GDMS to the quantitative analysis of tripeptide Gly-His-Lys copper complex (GHK-Cu). GHK is a naturally occurring peptide present in human plasma, saliva and urine. It has a strong affinity for copper and easily forms a highly stable monomeric copper complex in solution. ${ }^{20-21}$ It is believed that GHK fulfils its function as the copper complex and facilitates copper uptake into the cell. It is well recognized for its growth modulating functions as found in 
accelerating wound healing, stimulating hair growth, facilitating tissue repair, as well as anti-inflammatory and antioxidant effects. ${ }^{22}$ In recent years, the anti-cancer activity of GHK on early-stage sporadic colorectal cancer was explored and its therapeutic effect in reversing gene expression has been reported. ${ }^{23-24}$ Methods for quantitative analysis of GHK-Cu are of great significance for studies of its biological functions and future applications. Although tandem mass spectrometry (MS) based on soft ionization is more common for protein and peptide quantification, elemental mass spectrometry (MS) is attracting increased interest for its spectacular performance in quantitative analysis. ${ }^{1}$ Unlike tandem MS relying on the use of isotope-labelled peptides as internal standards, elemental MS simply provides accurate quantification of biomolecules through the determination of naturally occurring or artificially tagged elements under the premise of known stoichiometry. ${ }^{25-26}$

Pulsed RF glow discharge time-of-flight mass spectrometry, a powerful tool for quantitative determination $27-29$ was explored for its potential in GHK-Cu quantification. As mentioned above, GHK forms a 1:1 complex with $\mathrm{Cu}(\mathrm{II})$ ions in solution. ${ }^{21}$ Based on the known stoichiometric ratio of copper element to the tripeptide, quantitative analysis of GHK was accomplished through the measurement of copper content. Ion signals arising from the peptide-metal complex were evaluated at different times in the pulse cycle to identify characteristic ion signals with potential for quantitative use. GD operating conditions, including sampling distance, pressure, power and pulse parameters, were evaluated for their influences on the GHK-Cu characteristic ion peaks. Quantification was attempted with a set of peptide standards at different concentrations. 


\subsection{Experimental}

\subsubsection{Pulsed Radio Frequency Glow Discharge Time-of-Flight Mass}

\section{Spectrometry}

A six-way, high vacuum across (MDC Vacuum Products Co., Hayward, CA) serves as the ion source where GD ionization occurs. The plasma is powered by a 13.56 $\mathrm{MHz}$ radio frequency $(\mathrm{RF})$ generator equipped with an automatic matching network (Model RF 10-S, RF Plasma Product Inc., Marlton, NJ). The power supply operated under pulsed mode allows control of applied power, pulse width and duty cycle. A linear time-of-flight mass spectrometer (R.M. Jordan Co., Grass valley CA) built in house is coupled orthogonally to the GD chamber. This orientation allows pulsed injection of ions into the flight tube by pulsing the repeller plate in the ion extraction region. Application of the repeller voltage is triggered by a pulse generated with a variable delay relative to the pulse applied to the discharge. Adjustment of the repeller pulse delay permits selective injection of ions formed in any time regimes of a pulsed plasma. More information about the instrument configuration and data acquisition can be found in previous papers..$^{30-31}$

Samples mounted on the tip of a direct insertion probe (DIP) serves as GD cathode, and is introduced into the GD chamber through a ball valve. Cathodic sputtering is limited to the cathode front surface specifically by means of surrounding the cathode disk with a nonconducting ceramic shield machined from MACOR (Accuratus, Washington, N.J.). Sampling distance refers to the distance from the front 
surface of the sample cathode to the ion exit orifice. It can be adjusted by moving the DIP position horizontally in the GD chamber and was controlled within the range of 7-21 $\mathrm{mm}$ in this study. The GD power parameters (peak power, pulse width, duty cycle) was studied in the range of $70-130 \mathrm{~W}, 1-5 \mathrm{~ms}$ and $10-30 \%$ respectively. Argon (Ultra high purity, Matheson, Basking Ridge, $\mathrm{NJ}$ ) was used as the discharge gas and introduced to the GD chamber through a metering valve. Argon pressure in the GD chamber was monitored by a thermocouple pressure gauge (Varian, Lexington, MA) and controlled in the range of 400-900 mTorr. Detailed GD operation conditions were provided with mass spectra shown. Table 3.1 shows the summarized pulsed RF glow discharge time-of-flight mass spectrometer operating conditions.

\section{Table 3.1 Pulsed RF GD TOF-MS operating parameters}

\section{Glow discharge}

Pressure

Operation power

Pulse duration

Pulse duty cycle

Sampling distance

\section{Time-of-Flight}

Flight path length

\section{Ion lenses potential}

Skimmer

Extractor

Accelerator

Deflector (X1)

Deflector (Y1)

Repeller

$$
\begin{aligned}
& 400-900 \mathrm{mTorr} \\
& 70-130 \mathrm{~W} \\
& 1-5 \mathrm{~ms} \\
& 10 \%-30 \% \\
& 7-21 \mathrm{~mm}
\end{aligned}
$$

\section{$1 \mathrm{~m}$}

$$
-375 \mathrm{~V}
$$

$0 \mathrm{~V}$

$-1530 \mathrm{~V}$

$-1880 \mathrm{~V}$

$-1480 \mathrm{~V}$

$+250 \mathrm{~V}$ 


\begin{tabular}{lc}
\hline \multicolumn{1}{c}{ Table 3.1 Pulsed RF GD TOF-MS operating parameters (continued) } \\
\hline Detector & $-1895 \mathrm{~V}$ \\
Vacuum conditions & \\
Intermediate stage & $10^{-5}$ Torr \\
Flight tube & $10^{-6}$ Torr \\
\hline
\end{tabular}

\subsubsection{Preparation of GHK-Cu Solutions and Residues}

Glycyl-L-histidyl-L-lysine tripeptide copper complex (99\%, Peptide Science, Costa Mesa, CA) was dissolved in deionized (DI) water (18 M $\Omega$, Barnstead NANOpure Infinity, Lake Balboa, CA) to prepare a stock solution of $80 \mu \mathrm{g} / \mu \mathrm{L}$. Calibration standards at a set of concentrations $(5,10,20,30,40 \mu \mathrm{g} / \mu \mathrm{L})$ were prepared from the stock solution through serial dilution with DI water. Cesium iodide (99.9\%, Aldrich Chem Co., Milwaukee, WI) was spiked into the analyte solutions as an internal standard at a concentration of $1.5 \mu \mathrm{g} / \mu \mathrm{L}$.

GHK-Cu solutions cannot be directly analyzed by GDMS, therefore they were introduced into the ion source as dry solution residues formed on a conducting cathode. Silver was the choice of cathode material. Ag cylindrical disks (6 mm in length, $4.5 \mathrm{~mm}$ in diameter) was machined from a silver rod (99.9\%, Alfa Aesar, Ward Hill, MA) to serve as host cathodes. GHK-Cu residues were prepared by depositing an aliquot of analyte solution to the front surface of the Ag disk and dried in a vacuum desiccator for $30 \mathrm{~min}$. The Ag disk with GHK-Cu residue on surface was then loaded to the DIP (Fig. 3.1) and inserted to GD source for mass spectrometric analysis. Evaluations of GD operating 
conditions employ residues produced from $10 \mu \mathrm{L}$ analyte solution at $40 \mu \mathrm{g} / \mu \mathrm{L}$, whereas quantitative measurements were achieved with residues dried from $7 \mu \mathrm{L}$ aliquots.

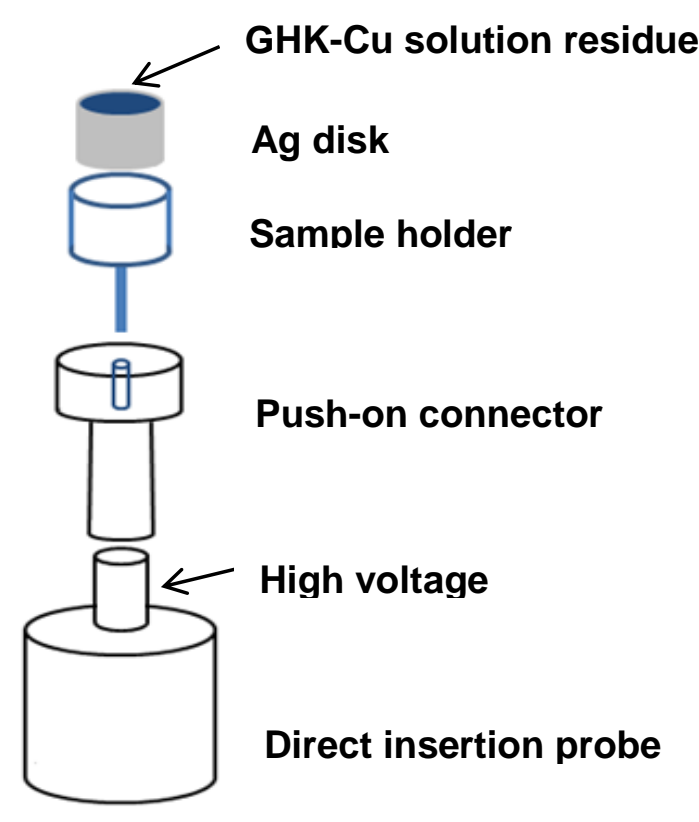

Figure 3.1 Schematic diagram of the sample introduction.

\subsection{Results and Discussion}

\subsubsection{Evaluation of Temporal Regimes}

In this work, the GD power was supplied as repeating square wave pulses, leading to the formation of three distinct temporal regimes: prepeak, plateau, afterpeak. In these three temporal regimes, different mechanisms dominate ionization in the plasma. When sampled in these regimes GHK-Cu species are expected to undergo different ionization process that can potentially reveal analyte information at different 
levels. For this reason, GHK-Cu spectra were collected from each temporal regime and inspected for any characteristic ion or unique fragmentation of the complex. For temporal evaluation studies, experimental conditions were kept consistent: 500 mTorr argon discharge gas, $100 \mathrm{~W}$ RF power, $2 \mathrm{~ms}$ pulse width, $10 \%$ duty cycle, and $12.5 \mathrm{~mm}$ sampling distance. The repeller time delay was varied to select a temporal region of interest.

Prepeak In the first several hundreds of microseconds, the GD plasma contains a large number of fast electrons; therefore, ions are formed mainly by collisions with electrons at this stage. Sputtering of the cathode surface by the positive argon ions or fast neutrals occurs shortly after the application of GD power, but the resultant atom or cluster liberated from the cathode surface is not immediately available for detection due to the time required for sputtering, diffusion, and ionization. This time delay is responsible for the absence of any analyte ion in the spectra collected in prepeak. Fig. 3.2 $\mathbf{a}, \mathbf{b}$ are the prepeak spectra collected at $0.3 \mathrm{~ms}$ for a blank Ag disk and a GHK-Cu residue loaded Ag disk (denoted as "GHK-Cu residue" hereinafter). As expected, both spectra do not contain any cathode-surface relevant peaks, i.e. $\mathrm{Ag}, \mathrm{GHK}-\mathrm{Cu}$, or any other related fragments. Only the discharge gas signals $\left(\mathrm{Ar}^{2+}, \mathrm{Ar}^{+}, \mathrm{ArH}^{+}, \mathrm{Ar}^{2+}\right.$ at $m / z 20$, $40,41,80$ respectively) and gas impurity signals $\left(\mathrm{OH}^{+}, \mathrm{H}_{2} \mathrm{O}^{+}\right.$at $\mathrm{m} / z 17,18$ respectively) appear.

Plateau. Mass spectra collected in the plateau of a pulsed GD exhibit the same characteristics as those collected from a continuous GD. Under pulsed operation, the plasma tends to stabilize about $1 \mathrm{~ms}$ after power initiation. During this period, the 
plasma is composed of a stable population of electrons, argon ions and metastable argon atoms. Excitation and ionization occur via a combination of electron impact, charge transfer and Penning ionization processes. Fig. $3.2 \mathrm{c}, \mathrm{d}$ are the plateau spectra collected at $1.9 \mathrm{~ms}$ for a blank $\mathrm{Ag}$ disk and $\mathrm{GHK}-\mathrm{Cu}$ residue. Background species remain in both spectra. $\mathrm{Ag}^{+}$signal arising from the supporting cathode appear in both spectra at $\mathrm{m} / \mathrm{z} 107$ and 109 with a naturally occurring isotopic ratio, but the sample spectrum shows a much lower intensity mostly due to the suppressed sputtering of the cathode surface by the residue. Minor peaks at $m / z 12,23$ are found in the spectrum of GHK-Cu complex (Fig. 3.2 d), which are attributed to ion signals of carbon and sodium (leftover from GHK-Cu synthesis process). In the plateau, the discharge plasma is well recognized to be highly energetic, with excitation and ionization usually accompanied by extensive collisional dissociation. ${ }^{32}$ This could possibly explain the absence of intact molecular ions or any large fragment ions of the GHK-Cu complex.

Afterpeak. Once the power is terminated, the GD plasma ceases the production of electrons and argon ions. The remaining fast electrons thermalize quickly through a series of collisions and recombine with argon ions, resulting an upsurge in the population of metastable argon atom. These metastable argon atoms possess an energy of $11.55 \mathrm{eV}$ or $11.72 \mathrm{eV}$, well above the ionization potentials of copper element and most peptide fragments. Consequently, Penning ionization now becomes the dominant ionization process for sputtered species. Owing to the increased number of Penning reagents available, an increased analytical signal can be obtained, seen clearly from the spectra (Fig. 3.2 e, f) collected at $2.3 \mathrm{~ms}$. In Fig. $\mathbf{3 . 2} \mathbf{f}, \mathrm{Ag}^{+}$ion signal is significantly enhanced, and its adducts with water also can be seen at $m / z 125 \& 127$. 
Residual sodium shows a much higher signal at $\mathrm{m} / \mathrm{z} 23$. Other than these, a distinct $\mathrm{Cu}^{+}$signal is observed at $\mathrm{m} / z 63 \& 65$, arising from dissociation of copper from the tripeptide-metal complex. Comparison of GHK-Cu spectra obtained from three temporal regimes suggests that $\mathrm{Cu}^{+}$afforded in afterpeak regime is the only characteristic ion found for the copper peptide. A further investigation of this $\mathrm{Cu}^{+}$ion signal in the afterpeak regime reveals that the best sensitivity can be obtained at $2.35 \mathrm{~ms}$, when the population of metastable argon atom is suggested to have reached its highest value. ${ }^{33}$

Although Penning ionization is believed to be a soft ionization method, no molecular ion or large fragments of the tripeptide are found. However, in a similar study, GD was reported to produce molecular and fragment ion signals from sucrose and tyrosine residues. ${ }^{15}$ Therefore, the missing of GHK-Cu molecular information indicates that extensive collision and dissociation of the tripeptide takes place during desorption or in the plasma prior to ionization.

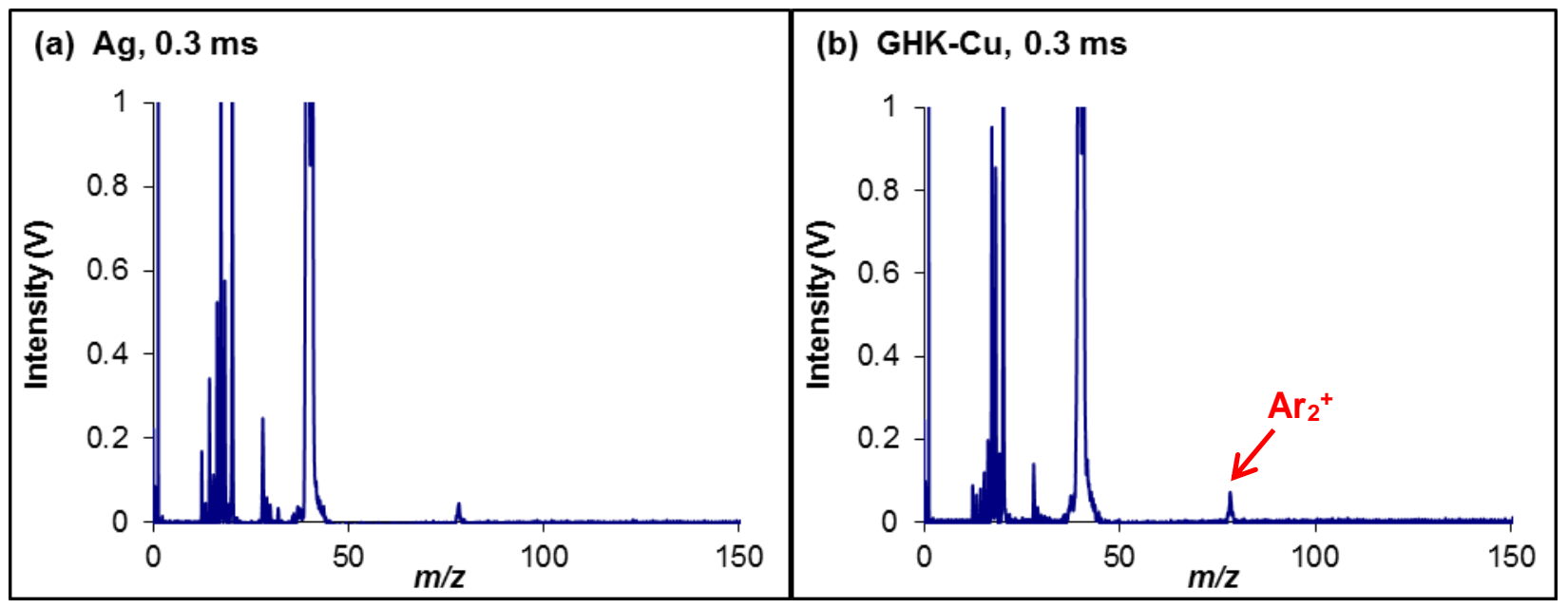



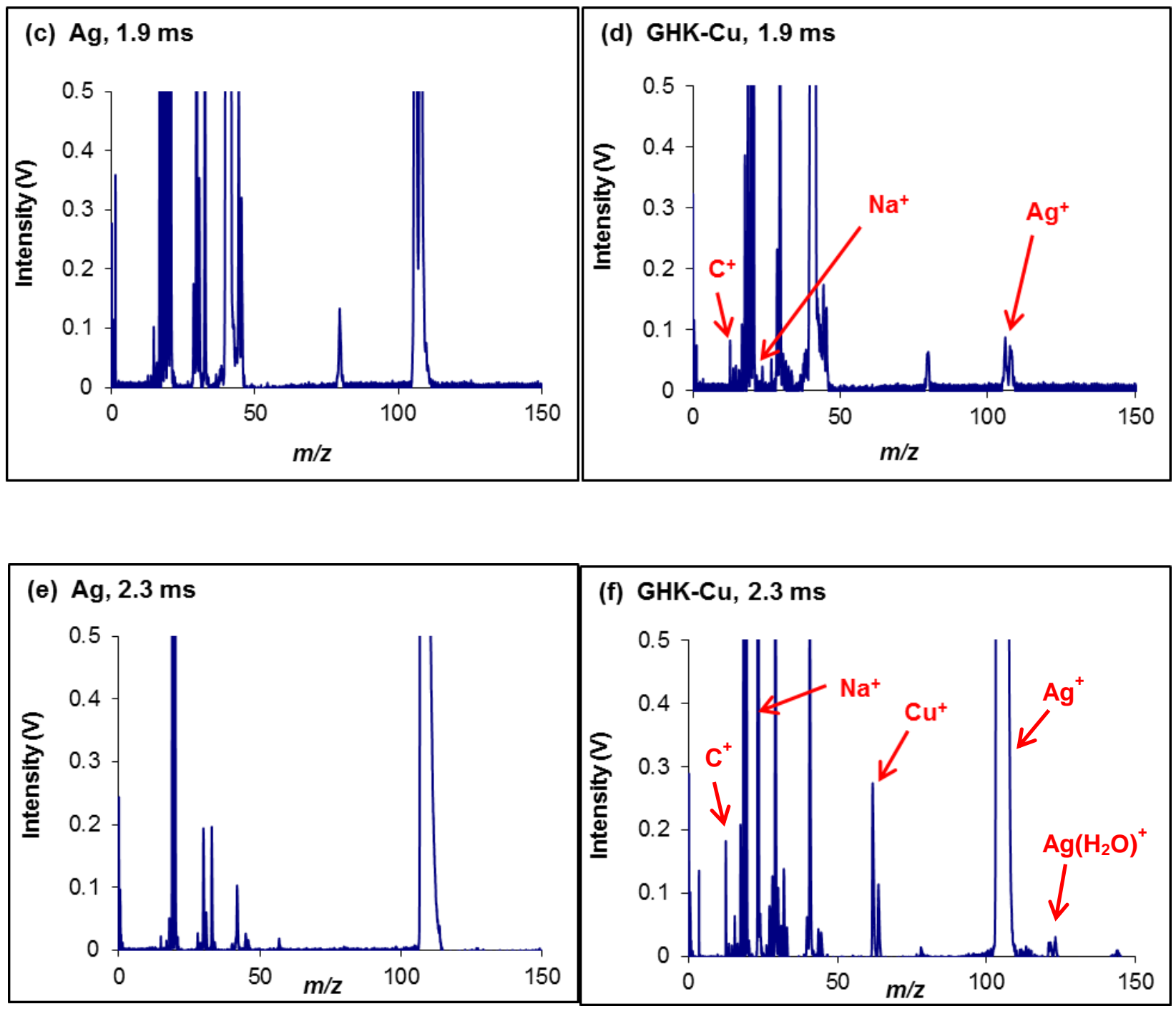

Figure 3.2 Time-resolved mass spectra for Ag cathode (as a blank for comparison) (left) and GHK-Cu residue on silver (right) collected at prepeak (0.3 ms) (a, b), plateau (1.9 ms) (c, d) and afterpeak (2.3 ms) (e, f). GD operating conditions: 500 mTorr argon discharge pressure, $100 \mathrm{~W}$ RF power, 2 ms pulse width, 10\% duty cycle and $12 \mathrm{~mm}$ sampling distance. 


\subsubsection{Evaluation of Spatial Variation}

Sampling distance controls the plasma region from which ions are sampled. By moving the DIP and sampling different regions, one can further control the ionization process responsible for the observed mass spectral signals. The evaluation of the sampling distance effect on the $\mathrm{Cu}^{+}$characteristic ion intensity, provides insight into both the ionization and dissociation of the GHK-Cu complex. The $\mathrm{Cu}^{+}$ion signal is barely visible when distance is lower than $7 \mathrm{~mm}$ or higher than $21 \mathrm{~mm}$. So, the signal change was closely monitored in the distance range of $7-21 \mathrm{~mm}$, as shown in Fig. 3.4. The $\mathrm{Cu}^{+}$ ion intensity exhibits a bell-shaped change over this distance range. As the cathode moves away from the ion sampling orifice to increase sampling distance, the $\mathrm{Cu}^{+}$ion signal first encounters an increase in intensity, reaches highest intensity at $15 \mathrm{~mm}$ and then declines at greater distances. Comparing to the studies of metallic copper, ${ }^{34}$ the maximal $\mathrm{Cu}^{+}$ion intensity from $\mathrm{GHK}-\mathrm{Cu}$ residue is obtained at a longer distance. We attribute this difference to the distinct nature of the analyte. In $\mathrm{GHK}-\mathrm{Cu}$ complex, $\mathrm{Cu}$ atom in each unit is bound to 3 nitrogens. ${ }^{20}$ In GD sputtering of GHK-Cu, a mixture of intact molecules, large fragments and individual atoms are expected to be released. Dissociation of the sputtered species to release the $\mathrm{Cu}$ atoms for ionization would maximize in the most energetic region of the plasma. 


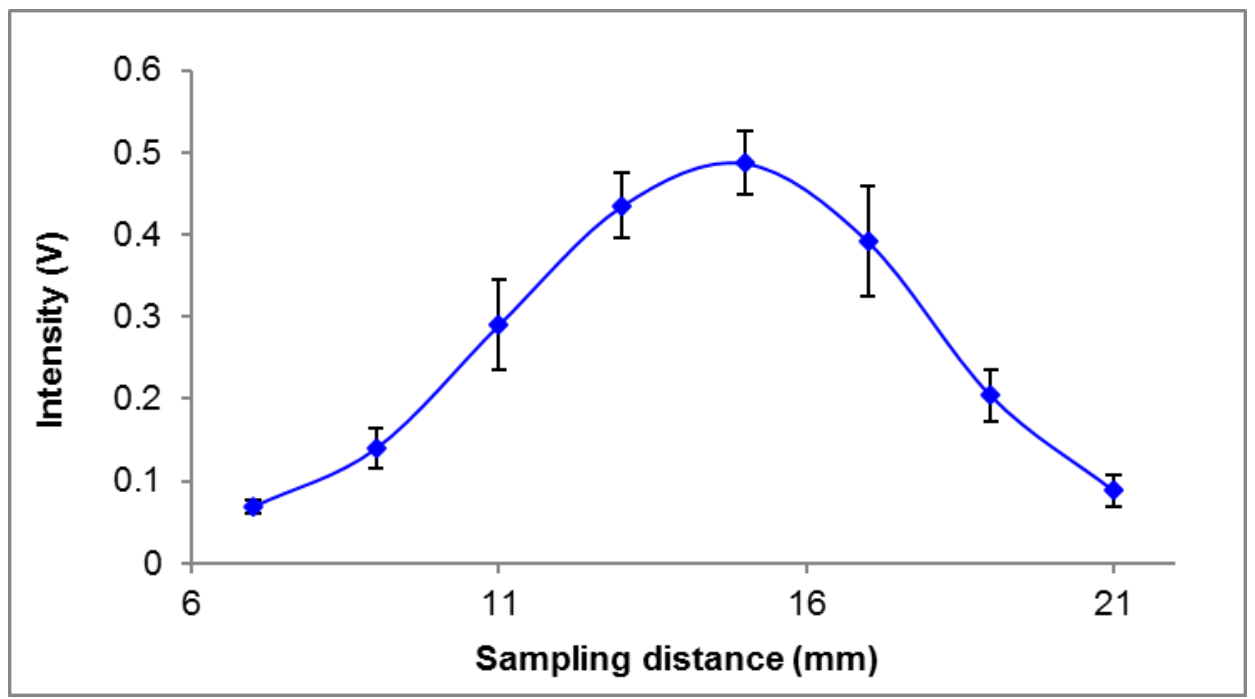

Figure 3.3 Spatial effect on ${ }^{63} \mathrm{Cu}^{+}$ion intensity profile collected at $2.35 \mathrm{~ms}$. GD operating conditions: 600 mTorr argon discharge pressure, $100 \mathrm{~W}$ RF power, $2 \mathrm{~ms}$ pulse width, $10 \%$ duty cycle. Each data point is the averaged value obtained from 3 replicates and error bars represent one standard deviation from the mean value.

\subsubsection{Optimization of Glow Discharge Operating Parameters}

Optimization of GD operating parameters has a significant effect on improving analytical signal and facilitating accurate measurement. The following experiments were undertaken to evaluate the influences of four parameters, including argon gas pressure, RF power, pulse width and duty cycle. Each parameter was varied separately to monitor the resulting changes in the $\mathrm{Cu}^{+}$ion intensity. Spectra were collected at 2.35 $\mathrm{ms}$ into the pulse cycle at a sampling distance of $15 \mathrm{~mm}$. In case of the pulse width study, ion signals were collected at $0.35 \mathrm{~ms}$ after pulse termination regardless of the pulse length. 
Pressure. Pressure of the discharge support gas, representative of the argon atom density, determines the mean free path and collision frequency of the analyte in the gas phase. A low-pressure plasma is characterized with low argon density and long mean free path, whereas a high-pressure plasma of high gas density would induce frequent collisions and thus increased fragmentation. Investigation of the pressure influences from 400 mTorr to 900 mTorr (Fig. 3.4 a) reveals that a highest ion signal can be obtained at 600 mTorr. Initial increase of pressure supplies a higher population of sputtering and ionization agents to the plasma, contributing to an enhanced analytical signal. A maximal signal was found at 600 mTorr, where the maximized Penning ionization efficiency was discovered. ${ }^{35}$ Further increase of pressure leads to a decreasing analyte signal as a result of reduced sputtering and ionization. In general, GD plasma initiated at higher pressure is characterized with high current and low voltage. This environment affords a lower kinetic energy for the argon ions and thus lowers the sputtering yield. Also, collisional deexcitation of metastable argon is increased at higher pressure, so a lower number of Penning agents is available for ionization. The increase of pressure would also affect the GD plasma shape. It was visually seen and experimentally proved that the plasma is more compressed to the cathode surface at higher pressure. ${ }^{34}$ A decreased signal shall be expected at the same sampling distance.

RF Power. GD in pulsed operation easily applies significantly higher instantaneous power than that can be afforded in a continuous source, improving sputtering, excitation and ionization. RF Power influences on the $\mathrm{Cu}^{+}$ion intensity was evaluated by varying power from 70 to $130 \mathrm{~W}$, at a constant pressure of 600 mTorr, as shown in Fig. $\mathbf{3 . 4} \mathbf{~ b . ~ I t ~}$ 
can be seen that power increase from 70 to $90 \mathrm{~W}$ brings about a clear increase of signal intensity. Continuous increase from 90 to $110 \mathrm{~W}$ displays a slightly enhanced but more stable signal obtained from a more-stabilized plasma, as indicated by the smaller error bars. When the power is further increased, the $\mathrm{Cu}^{+}$ion signal gains intensity but is less reproducible due to plasma instability. Generally, in an abnormal GD, increase of power would induce increase in both current and voltage, which means a higher number of argon ions at higher kinetic energy will be created. So, enhanced sputtering and ionization in the plasma can be anticipated, leading to the overall increase of the $\mathrm{Cu}^{+}$ion intensity. In practice, conditions favoring a stable plasma are sought to provide consistent sputtering and ionization $-100 \mathrm{~W}$ was found to provide this stability.

Pulse Width and Duty Cycle. GD pulse width and duty cycle are critical parameters for pulsed operation, so each was varied independently to evaluate their impact on the $\mathrm{Cu}^{+}$ion signal with effective power held constant. Investigation of the two parameters' influences on the ion profile was conducted by varying the duty cycle at a fixed $2 \mathrm{~ms}$ pulse width (Fig. 3.4 c) and varying the pulse width at a fixed 10\% duty cycle (Fig. 3.4 d), while the frequency was allowed to reach its own value depending on the other two parameters. As seen in Fig. 3.4 (c, d), an intense $\mathrm{Cu}^{+}$ion signal is readily obtained at a shorter pulse and lower duty cycle. This observation is in agreement with the previous findings reported in this group. ${ }^{34}$ Reduced signal at longer pulse durations and higher duty cycles can be explained by gas heating effect as reported in the fundamental studies of GD electrical properties. ${ }^{36-37}$ At long pulse durations and high duty cycles, increased gas temperature arising from various collision processes, can cause a reduction in gas density and the resulting reduction in current will then affect the 
electrical properties of the discharge. GD plasma produced is expected to be more energetic and the argon ions containing high kinetic energy would disfavour the formation of metastable argon atom after power removal.
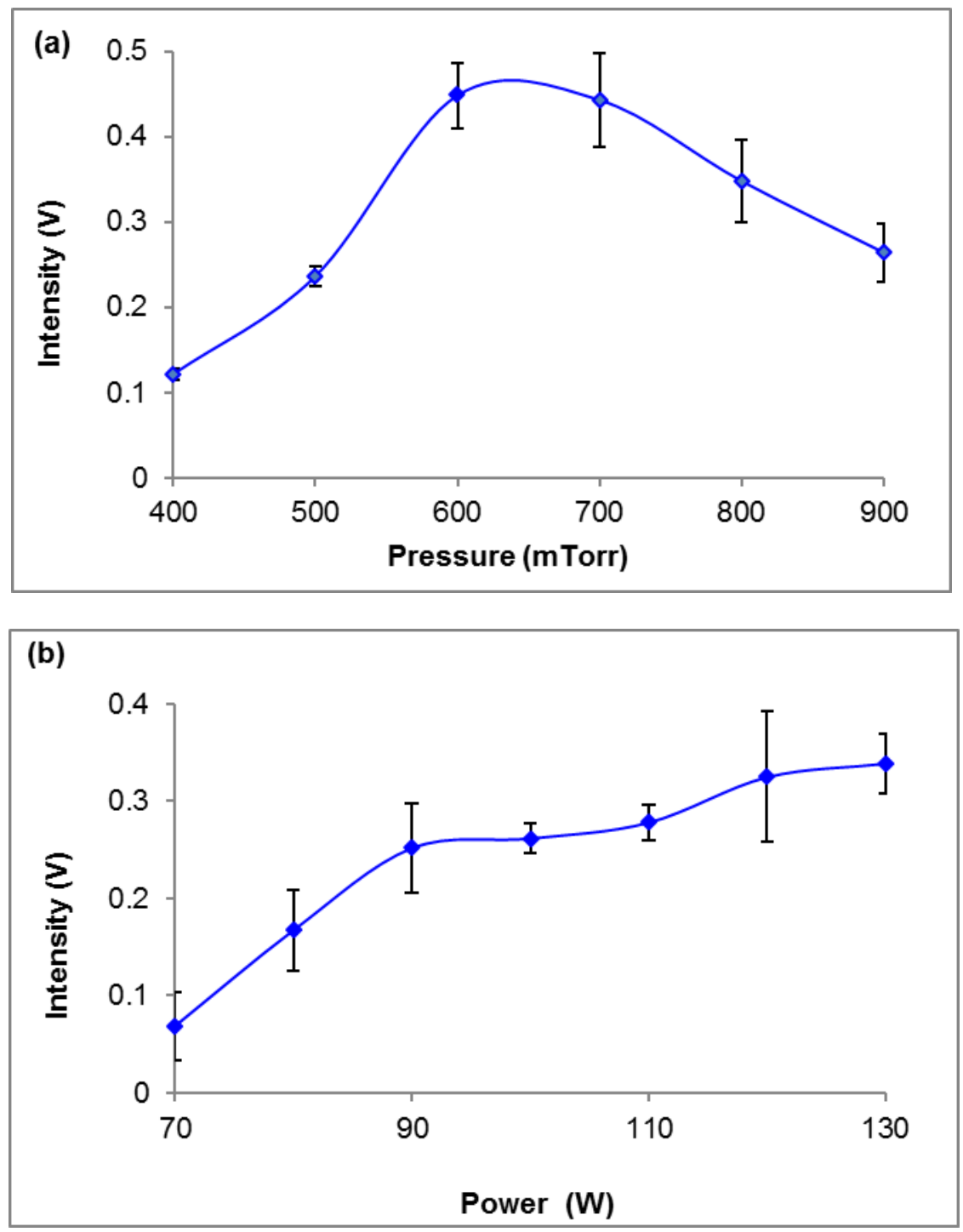

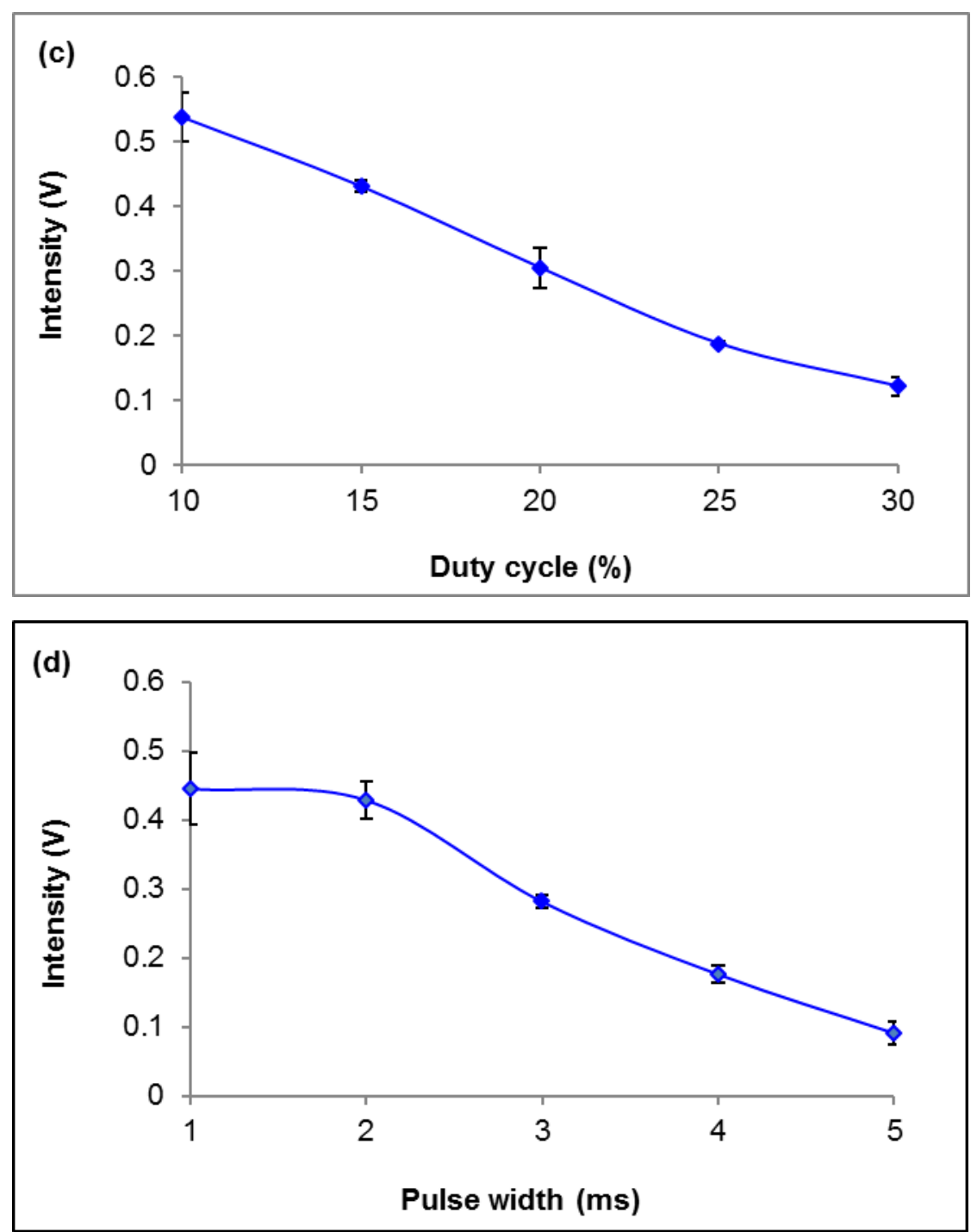

Figure 3.4 Influences of GD operating parameters on ${ }^{63} \mathrm{Cu}^{+}$ion intensity profile: (a) $\begin{array}{lll}\text { Pressure (b) RF Power (c) Duty cycle (fixed pulsed width } 2 \mathrm{~ms} \text { ) } & \text { (d) Pulse width (fixed }\end{array}$ duty cycle 10\%). Spectra are collected at $2.35 \mathrm{~ms}$ and $15 \mathrm{~mm}$ sampling distance. GD operating conditions are kept consistent at 600 mTorr argon discharge pressure, 100W RF power, 2 ms pulse width, $10 \%$ duty cycle unless the parameter is varied as $\mathrm{x}$-axis. Each data point is the averaged value obtained from 3 replicates and error bars represent one standard deviation from the mean value. 


\subsubsection{Quantitative Analysis of GHK-Cu Complex}

Knowledge of the stoichiometry of the GHK-Cu complex allows the quantitative determination of the peptide to be completed with the measurement of the $\mathrm{Cu}^{+}$ion intensity. GHK-Cu residues were prepared by drying the same volume of copper peptide solutions from five concentrations. For each concentration, 4 replicates were prepared and used to obtain an averaged ion intensity. Using cesium iodide as an internal standard, intensity of the $\mathrm{Cu}^{+}$ion at $\mathrm{m} / \mathrm{z} 63$ was normalized to the $\mathrm{Cs}^{+}$ion intensity to account for any signal variations resulted from solution deposit variation or plasma instability.

Residue analysis starts upon GD initiation and stops when significant signal loss occurs, as shown in Fig. 3.5. First, it is apparent that the intensity of the $\mathrm{Cu}^{+}$ion signal is directly related to the amount of the GHK-Cu complex in the residue. Accompanied with an increasing concentration of the peptide solution, an increasing amount of GHK$\mathrm{Cu}$ complex can be found in the residue, leading to an increasing $\mathrm{Cu}^{+}$ion signal. The highest $\mathrm{Cu}+$ ion signal can be seen for the residue representing the highest concentration. Second, due to the limited quantity of GHK-Cu in the residue, the $\mathrm{Cu}^{+}$ characteristic ion signal decreases gradually over analysis time as the sputtering continuously strips analyte from the cathode surface. However, the $\mathrm{Cu}^{+}$ion signal trends do show variation for the five concentrations. For concentrations of $5,10 \mu \mathrm{g} / \mu \mathrm{L}$, the $\mathrm{Cu}^{+}$ion signal suffers slow decay due to continuous sample loss as sputtering proceeds, whereas for concentrations of $20,30,40 \mu \mathrm{g} / \mu \mathrm{L}$, the ion signal needs a short period to reach a steady state before it starts to decrease. 


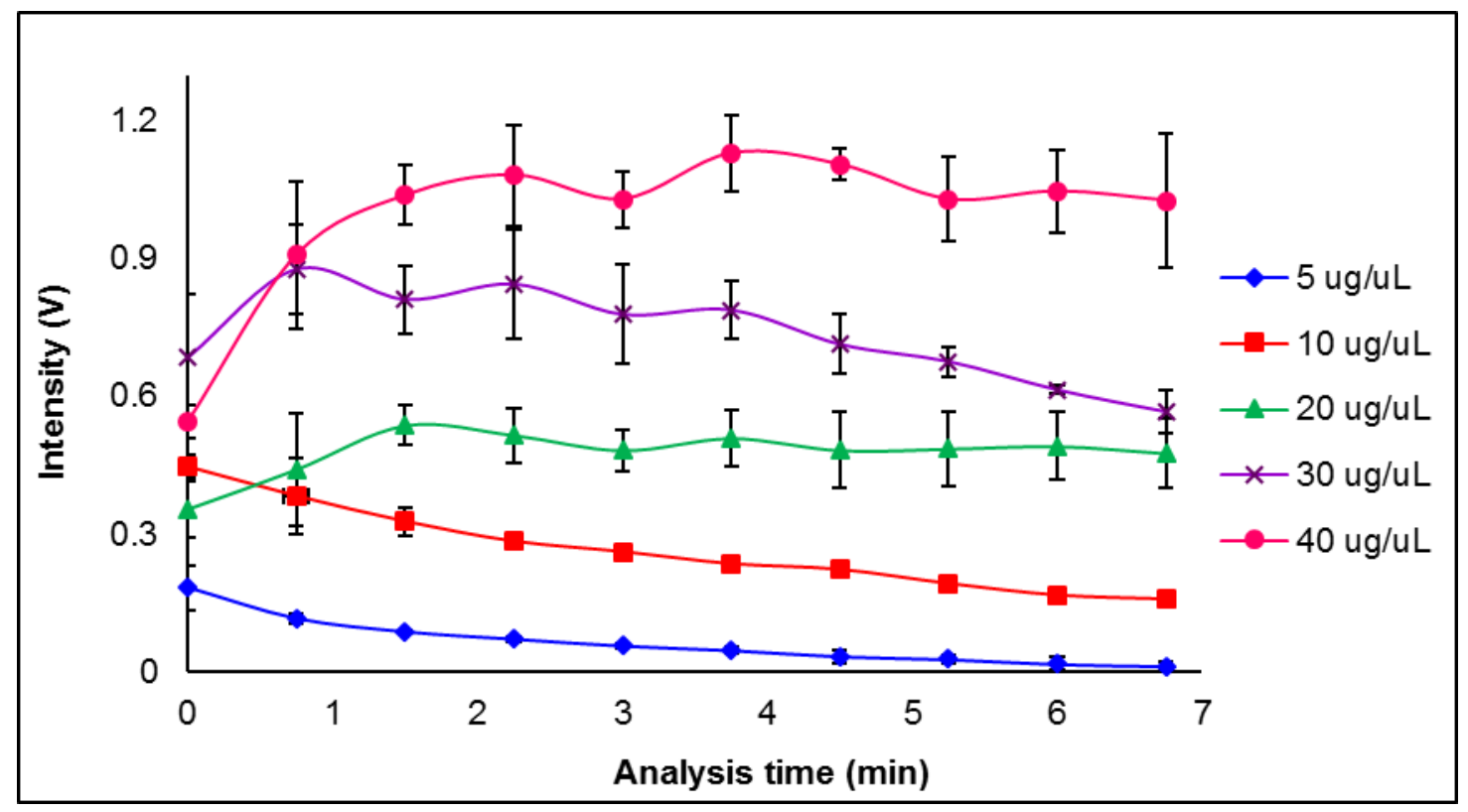

Figure $3.5{ }^{63} \mathrm{Cu}^{+}$ion intensity profile over sputtering time for solution residues of $7 \mu \mathrm{L}$. Spectra collected at 2. 35 ms with optimal GD operating conditions: $100 \mathrm{~W}$ RF power, 2 ms pulse width, $10 \%$ duty cycle, 600 mTorr argon discharge pressure, and $15 \mathrm{~mm}$ sampling distance. Each data point is the averaged value obtained from 4 replicates and error bars represent one standard deviation from the mean value.

After careful review of the signal trend for each concentration, the $\mathrm{Cu}^{+}$ion signal obtained at 3.75 min after power initiation was employed to construct the calibration curve of the GHK-Cu complex (Fig. 3.6). It turns out that the $\mathrm{Cu}^{+}$ion intensity correlates well with the peptide concentration and confirms the applicability of GDMS approach in the quantitative study of metal peptide complex. The calibration curve based on normalized copper ion intensity exhibits excellent linearity $(m=30.1 \mathrm{mV} / \mathrm{GHK}-\mathrm{Cu} \mu \mathrm{g} / \mu \mathrm{L}$, $\left.b=-91.4 \mathrm{mV}, R^{2}=0.9978\right)$ with a limit of quantification calculated from $10 \mathrm{~S} / \mathrm{N}$ determined to be $1.69 \mu \mathrm{g} / \mu \mathrm{L}$. 


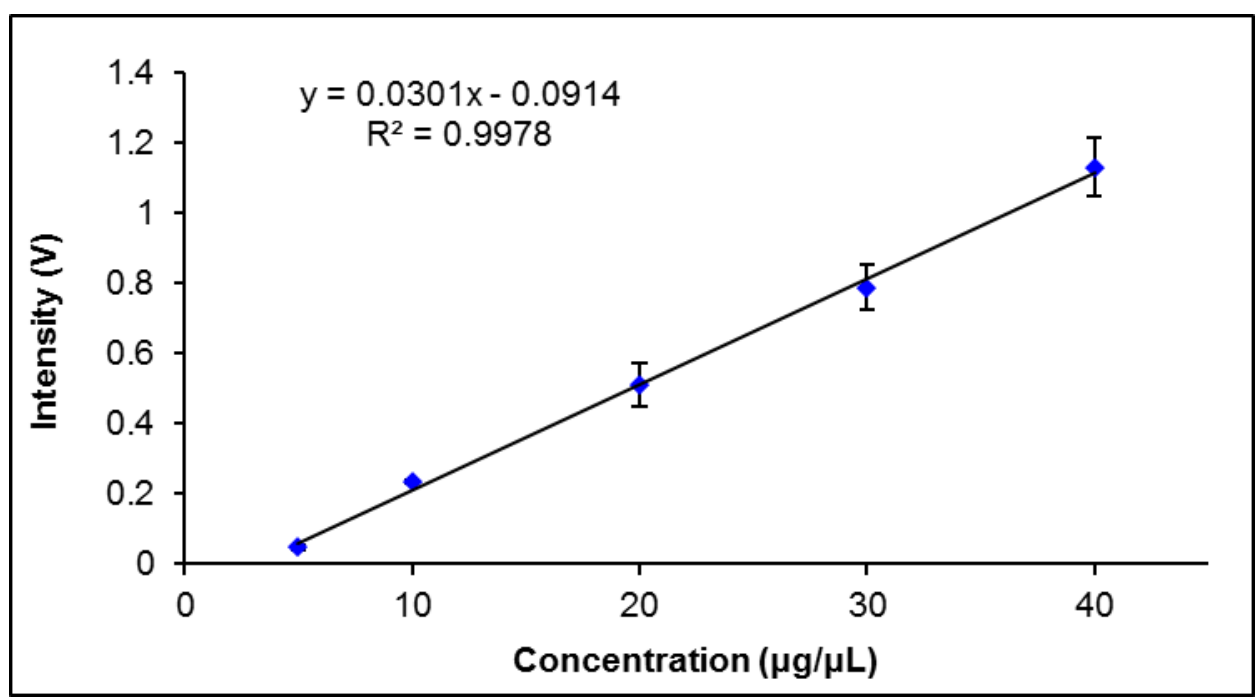

Figure 3.6 Calibration curve of ${ }^{63} \mathrm{Cu}^{+}$ion intensity (collected at 3.75 min from previous figure) vs. GHK-Cu solution concentration. Spectra collected at 2. $35 \mathrm{~ms}$ with optimal GD conditions: 100 W RF power, 2 ms pulse width, 10\% duty cycle, 600 mTorr argon gas, and $15 \mathrm{~mm}$ sampling distance. Each data point is the averaged value obtained from 4 replicates and error bars represent one standard deviation from the mean value.

\subsection{Conclusion}

GD operating parameters and sampling distance were carefully reviewed for their effects on the $\mathrm{Cu}^{+}$ion intensity arising from the GHK-Cu complex. Based on the these results, optimal conditions were selected to perform the quantitative analysis of the peptide complex: $100 \mathrm{~W}$ RF power, $15 \mathrm{~mm}$ sampling distance, $600 \mathrm{mTorr}$ argon pressure, 2 ms pulse width and $10 \%$ duty cycle. The $\mathrm{Cu}^{+}$ion intensities from GHK-Cu solutions at 5 concentrations were used to build the calibration curve, which showed excellent linearity. 
This work demonstrates the applicability of GDMS in the quantitative analysis of a metal-associated peptide complex via the metal. The copper peptide is sampled by a GD plasma as solution residue deposited on cathode surface. Based on known stoichiometry, GHK quantification is achieved through measurements of the copper ion signal during the afterpeak regime. This quantitative approach is limited by the performance of the home built GDMS system's poor sensitivity. Commercial instruments of high performance should allow the quantification to be completed with less amount of sample over a wide concentration range, affording low limit of detection and quantification. Overall, this study demonstrates proof of the concept of GDMS application in quantitative determination of metal associated peptides through elemental detection. Future work should extend the application of this GDMS approach to the quantification of other metal containing peptide/protein via metal detection. 


\subsection{References}

1. Yan, X.; Yang, L.; Wang, Q., Detection and quantification of proteins and cells by use of elemental mass spectrometry: progress and challenges. Analytical and bioanalytical chemistry 2013, 405 (17), 5663-70.

2. Pereiro, R.; Solà-Vázquez, A.; Lobo, L.; Pisonero, J.; Bordel, N.; Costa, J. M.; Sanz-Medel, A., Present and future of glow discharge - Time of flight mass spectrometry in analytical chemistry. Spectrochimica Acta Part B: Atomic Spectroscopy 2011, 66 (6), 399-412.

3. Bouza, M.; Pereiro, R.; Bordel, N.; Sanz-Medel, A.; Fernandez, B., Pulsed radiofrequency glow discharge time of flight mass spectrometry for coated glass analysis. J. Anal. At. Spectrom. 2015, 30 (5), 1108-1116.

4. Gonzalez de Vega, C.; Fernandez, B.; Bordel, N.; Pereiro, R.; Sanz-Medel, A., Challenging identifications of polymer coatings by radiofrequency pulsed glow discharge-time of flight mass spectrometry. J. Anal. At. Spectrom. 2013, 28 (7), 10541060.

5. Lobo, L.; Fernandez, B.; Muniz, R.; Pereiro, R.; Sanz-Medel, A., Capabilities of radiofrequency pulsed glow discharge-time of flight mass spectrometry for molecular screening in polymeric materials: positive versus negative ion mode. J. Anal. At. Spectrom. 2016, 31 (1), 212-219.

6. Fernandez, B.; Lobo, L.; Reininghaus, N.; Pereiro, R.; Sanz-Medel, A., Characterization of thin film tandem solar cells by radiofrequency pulsed glow discharge

- Time of flight mass spectrometry. Talanta 2017, 165, 289-296. 
7. Modanese, C.; Arnberg, L.; Di Sabatino, M., Analysis of impurities with inhomogeneous distribution in multicrystalline solar cell silicon by glow discharge mass spectrometry. Materials Science and Engineering: B 2014, 180, 27-32.

8. Guzowski Jr, J. P.; Hieftje, G. M., Characterization of switched direct current gas sampling glow discharge ionization source for the time-of-flight mass spectrometer. J. Anal. At. Spectrom. 2000, 15 (1), 27-36.

9. Lewis, C. L.; Moser, M. A.; Dale Jr, D. E.; Hang, W.; Hassell, C.; King, F. L.; Majidi, V., Time-gated pulsed glow discharge: real-time chemical speciation at the elemental, structural, and molecular level for gas chromatography time-of-flight mass spectrometry. Anal. Chem. 2003, 75 (9), 1983-96.

10. Bouza, M.; Fandino, J.; Bordel, N.; Pereiro, R.; Sanz-Medel, A., Volatile organic compounds analysis by pulsed glow discharge time of flight mass spectrometry as a structural elucidation tool. J. Mass Spectrom. 2017.

11. Harrison, W. W.; Hang, W. E. I.; Yan, X.; Ingeneri, K.; Schilling, C., Temporal Considerations With a Microsecond Pulsed Glow Discharge. J. Anal. At. Spectrom. 1997, 12 (9), 891-896.

12. Marcus, R. K.; Broekaert, J. A., Glow discharge plasmas in analytical spectroscopy. John Wiley \& Sons: 2003.

13. Barshick, C. M.; Duckworth, D. C.; Smith, D. H., Analysis of solution residues by glow discharge mass spectrometry. J. Am. Soc. Mass. Spectrom. 1993, 4 (1), 47-53.

14. Jakubowski, N.; Stuewer, D.; Toelg, G., Microchemical determination of platinum and iridium by glow-discharge mass spectrometry. Spectrochim. Acta, Part B 1991, $46 B$ (2), 155-63. 
15. Mason, R.; Milton, D., Glow discharge mass spectrometry of some organic compounds. Int. J. Mass Spectrom. Ion Processes 1989, 91 (2), 209-25.

16. Scott, R. P. W.; Scott, C. G.; Munroe, M.; Hess, J., Interface for on-line liquid chromatography_mass spectroscopy analysis. J. Chromatogr. A 1974, 99, 395-405.

17. Brewer, T. M.; Castro, J.; Marcus, R. K., Particle beam sample introduction into glow discharge plasmas for speciation analysis. Spectrochimica Acta Part B: Atomic Spectroscopy 2006, 61 (2), 134-149.

18. Zhang, L. X.; Marcus, R. K., Mass spectra of diverse organic species utilizing the liquid sampling-atmospheric pressure glow discharge (LS-APGD) microplasma ionization source. J. Anal. At. Spectrom. 2016, 31 (1), 145-151.

19. Schwartz, A. J.; Shelley, J. T.; Walton, C. L.; Williams, K. L.; Hieftje, G. M., Atmospheric-pressure ionization and fragmentation of peptides by solution-cathode glow discharge. Chemical Science 2016, 7 (10), 6440-6449.

20. Pickart, L.; Freedman, J. H.; Loker, W. J.; Peisach, J.; Perkins, C. M.; Stenkamp, R. E.; Weinstein, B., Growth-modulating plasma tripeptide may function by facilitating copper uptake into cells. Nature 1980, 288 (5792), 715-717.

21. Freedman, J. H.; Pickart, L.; Weinstein, B.; Mims, W. B.; Peisach, J., Structure of the glycyl-L-histidyl-L-lysine-copper(II) complex in solution. Biochemistry 1982, 21 (19), 4540-4544.

22. Pickart, L., The human tri-peptide glycine-histidine-lysine and tissue remodeling. J. Biomater. Sci., Polym. Ed. 2008, 19 (8), 969-988.

23. Hong, Y.; Downey, T.; Eu, K. W.; Koh, P. K.; Cheah, P. Y., A 'metastasis-prone' signature for early-stage mismatch-repair proficient sporadic colorectal cancer patients 
and its implications for possible therapeutics. Clinical \& experimental metastasis 2010, 27 (2), 83-90.

24. Campbell, J. D.; McDonough, J. E.; Zeskind, J. E.; Hackett, T. L.; Pechkovsky, D. V.; Brandsma, C.-A.; Suzuki, M.; Gosselink, J. V.; Liu, G.; Alekseyev, Y. O.; Xiao, J.; Zhang, X.; Hayashi, S.; Cooper, J. D.; Timens, W.; Postma, D. S.; Knight, D. A.; Marc, L. E.; James, H. C.; Avrum, S., A gene expression signature of emphysema-related lung destruction and its reversal by the tripeptide GHK. Genome Med. 2012, 4 (8), 67.

25. Sanz-Medel, A.; Montes-Bayón, M.; Bettmer, J.; Luisa Fernández-Sanchez, M.; Ruiz Encinar, J., ICP-MS for absolute quantification of proteins for heteroatom-tagged, targeted proteomics. TrAC, Trends Anal. Chem. 2012, 40, 52-63.

26. Liu, Z.; Li, X.; Xiao, G.; Chen, B.; He, M.; Hu, B., Application of inductively coupled plasma mass spectrometry in the quantitative analysis of biomolecules with exogenous tags: A review. TrAC, Trends Anal. Chem. 2017, 93, 78-101.

27. Matschat, R.; Hinrichs, J.; Kipphardt, H., Application of glow discharge mass spectrometry to multielement ultra-trace determination in ultrahigh-purity copper and iron: a calibration approach achieving quantification and traceability. Analytical and bioanalytical chemistry 2006, 386 (1), 125-41.

28. Alvarez-Toral, A.; Fernandez, B.; Malherbe, J.; Claverie, F.; Molloy, J. L.; Pereiro, R.; Sanz-Medel, A., Isotope dilution mass spectrometry for quantitative elemental analysis of powdered samples by radiofrequency pulsed glow discharge time of flight mass spectrometry. Talanta 2013, 115, 657-664.

29. Ganeev, A.; Bogdanova, O.; Ivanov, I.; Burakov, B.; Agafonova, N.; Korotetski, B.; Gubal, A.; Solovyev, N.; lakovleva, E.; Sillanpaa, M., Direct determination of uranium 
and thorium in minerals by time-of-flight mass spectrometry with pulsed glow discharge. RSC Advances 2015, 5 (99), 80901-80910.

30. Steiner, R. E.; Lewis, C. L.; King, F. L., Time-of-Flight Mass Spectrometry with a Pulsed Glow Discharge Ionization Source. Anal. Chem. 1997, 69 (9), 1715-21.

31. Zhang, N.; King, F. L., Direct manganese (Mn) speciation in solid state materials by pulsed glow discharge time-of-flight mass spectrometry. J. Anal. At. Spectrom. 2009, $24(11), 1489-1497$.

32. Li, L.; Millay, J. T.; Turner, J. P.; King, F. L., Millisecond pulsed radio frequency glow discharge time of flight mass spectrometry: temporal and spatial variations in molecular energetics. J. Am. Soc. Mass. Spectrom. 2004, 15 (1), 87-102.

33. King, F. L.; Pan, C., Temporal signal profiles of analytical species in modulated glow discharge plasmas. Anal. Chem. 1993, 65 (6), 735-739.

34. DeJesus, M. R.; Gu, G.; King, F. L.; Barnes, J. H.; Lewis, C. L., Ion formation in millisecond pulsed glow discharge plasmas. J. Anal. At. Spectrom. 2011, 26 (11), 22062215.

35. Smith, R. L.; Serxner, D.; Hess, K. R., Assessment of the relative role of Penning ionization in low-pressure glow discharges. Anal. Chem. 1989, 61 (10), 1103-1108.

36. Hoffmann, V.; Efimova, V. V.; Voronov, M. V.; Šmíd, P.; Steers, E. B. M.; Eckert, J., Measurement of voltage and current in continuous and pulsed if and dc glow discharges. Journal of Physics: Conference Series 2008, 133 (1), 012017.

37. Efimova, V.; Hoffmann, V.; Eckert, J., Electrical properties of the $\mu$ s pulsed glow discharge in a Grimm-type source: comparison of dc and if modes. J. Anal. At. Spectrom. 2011, 26 (4), 784-791. 


\section{Chapter 4}

\section{Glow Discharge Approach for Quantitative Determination of lodinated Organic Compounds}

\section{Abstract}

Pulsed glow discharge mass spectrometry (GDMS) was applied to the analysis of three iodinated organic compounds from the thyroid hormone (TH) family. $\mathrm{TH}$ compounds were introduced to glow discharge (GD) plasma as dry solution residues generated on the cathode surface. A helium plasma was successfully established within the GDMS system and affords favorable sputtering and ionization efficiency in a sample test of $\mathrm{Cu}$ disk. Analysis of triiodothyronine (T3) in $\mathrm{He}$ and Ar plasma both reveals a distinct iodine ion signal in the afterpeak regime, and comparison of the iodine ion intensities between the two plasmas further confirms the analyzing efficiency of $\mathrm{He}$ plasma. Analysis of THs' behaviors over a pulse cycle displays a similar pattern, and the only signal that can be identified is the iodine ion signal produced in the afterpeak for all THs. Quantification based on the afterpeak iodine ion signal was demonstrated using diiodothyronine (T2) as an example and a calibration curve that correlates the iodine ion intensity with the solution concentration displays good linearity. Cross comparison among three THs were made by analyzing residues containing the same molar amount of each compound, and the proportional increase relationship of the 
acquired iodine ion signals indicates that the iodine intensity is independent of its position within a molecule, but only relating to its amount.

\subsection{Introduction}

lodine is an essential substrate for the production of thyroxine (T4) and triiodothyronine (T3). These thyroid hormones (THs) regulate a number of biological processes in cellular metabolism, body growth, neuronal function and development. ${ }^{1}$ Because the human body does not produce iodine, dietary iodine intake is essential to maintain the thyroid hormone levels as needed for normal body functions. lodine deficiency can cause multiple adverse effects on growth and development, including enlargement of thyroid gland, hypothyroidism, irreversible mental retardation, etc. These effects are now summarized in the term "iodine deficiency disorder" (IDD). ${ }^{2}$ However, excessive intake of iodine is also detrimental to human body because it inhibits iodine uptake by the thyroid gland and subsequently impairs the synthesis of thyroid hormones. ${ }^{3}$

lodine species exist in the environment in different forms depending on the matrix in which they are found. Inorganic species are chiefly found in aqueous matrices as iodide and iodate, whereas organic iodine compounds appear mostly in biological matrices. ${ }^{4}$ lodine circulation in the biosphere undergoes a number of complex physical, chemical and biological processes as shown in Fig. 4.1. This global cycle of iodine is 
essential to life because the majority of the iodine found on earth's crust is not accessible except for the small amounts liberated by weathering and dissolution. ${ }^{5}$

Several approaches are available for the determination of iodine levels in environmental, biological and clinical materials. Classical spectrophotometric methods involve the detection of a color change arising either from the formation of a colored complex between iodine and starch, or a reduction in the color resulting from the iodide catalyzed Sandell-Kolthoff reaction. ${ }^{6}$ Other techniques, such as atomic absorption spectrometry (AAS), ${ }^{7}$ inductively coupled plasma-optical emission spectrometry (ICPOES), ${ }^{8-9}$ and inductively coupled plasma-mass spectrometry (ICP-MS), ${ }^{10}$ are also reported for iodine determination. Among these, ICP-MS is the most widely used because it offers the highest sensitivity. ICP-MS enables the determination of the total iodine content in aqueous samples including urine, blood serum, and milk ${ }^{11-13}$ as well as solid materials such as coal, sediment and seaweed. ${ }^{14-16}$ Speciation of individual iodine compounds requires the use of a separation technique like $L C^{17}$ and $C E^{18}$ prior to ICPMS determination. This combination has successfully accomplished speciation of both inorganic and organic iodine species found in seawater, rain, river and drinking water, ${ }^{19}$ body fluids, ${ }^{17-18}$ animal tissues ${ }^{20-21}$ and dog foods. ${ }^{22}$

The present study demonstrates the application of glow discharge mass spectrometry (GDMS) in the direct determination of iodinated organic compounds from the thyronine family. Three iodinated compounds including L-Thyroxine (T4), 3,3',5Triiodo-L-Thyronine (T3), and 3,5-Diiodo-L-Thyronine (T2) were analyzed by GDMS as dry solution residues. A plasma using helium as discharge gas was initiated in the 
GDMS system and employed in the analysis of $\mathrm{TH}$ residues. An intense iodine signal was discovered for all iodinated compounds in the afterglow regime of the helium glow discharge (GD). The intensity of this signal was then compared with that found in an argon glow discharge (GD) to assess the sputtering and ionization efficiency of a helium plasma. The feasibility of using iodine ion signal to provide quantitative measurements of iodinated compounds within the GDMS system was explored. Organic compounds containing different numbers of iodine atoms in a molecule were analyzed and the results were compared to assess any relationship between the iodine ion intensity and the number and location of iodine atoms within a molecule.

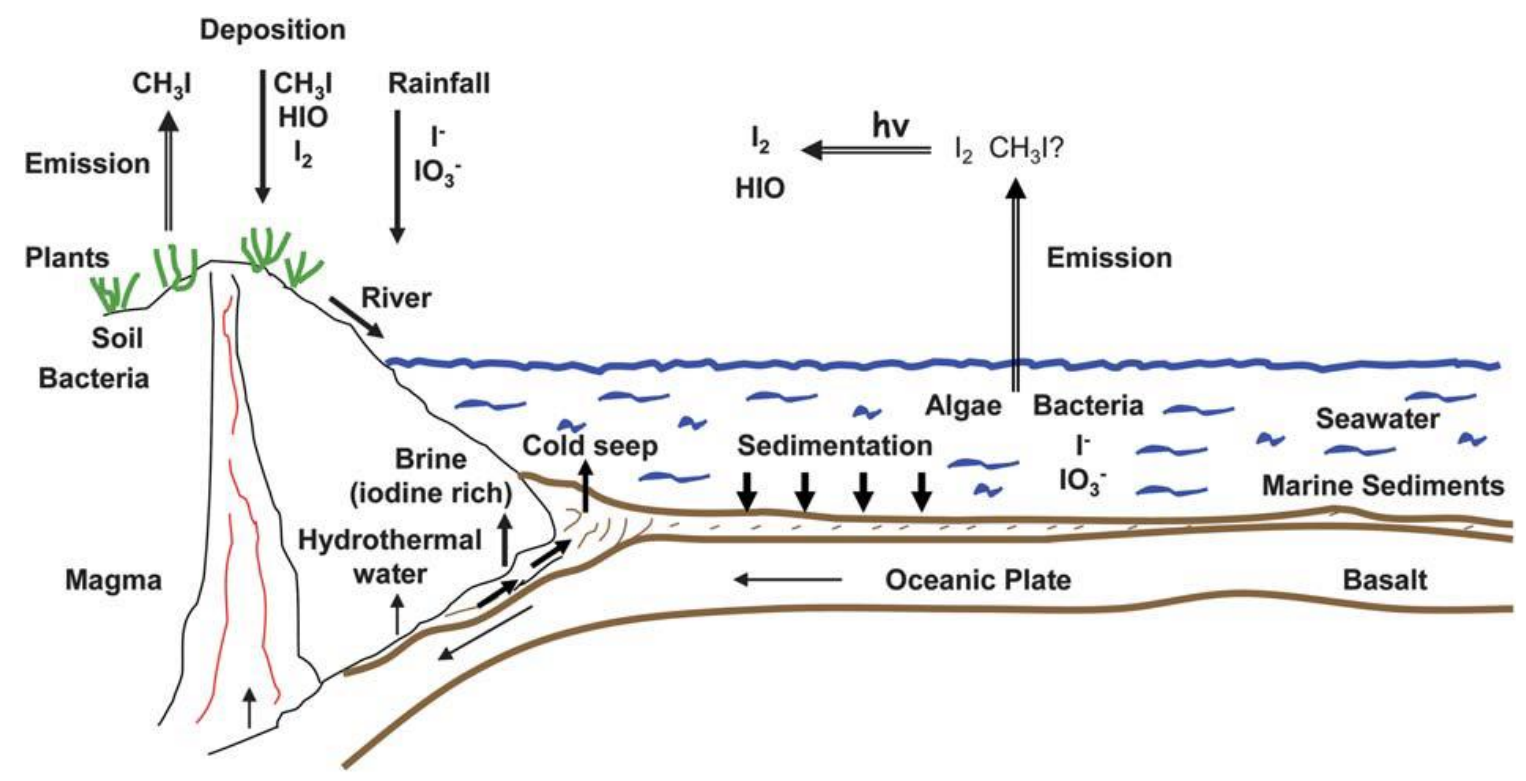

Figure 4.1 Global cycle of iodine. ${ }^{5}$ 


\subsection{Experimental}

\subsubsection{Pulsed Radio Frequency Glow Discharge Time-of-Flight Mass}

\section{Spectrometry}

A six-way, high vacuum across (MDC Vacuum Products Co., Hayward, CA) serves as the ion source where GD ionization occurs. A $13.56 \mathrm{MHz}$ radio frequency (RF) generator (Model RF 10-S, RF Plasma Product Inc., Marlton, NJ) equipped with an automatic matching network (Model AM-10, RF Plasma Product Inc., Marlton, NJ) provides power to operate the GD. Operation of this power supply in a pulsed mode allows control of applied power, pulse width and duty cycle. The GD ionization chamber interfaces orthogonally with the linear time-of-flight mass spectrometer (R.M. Jordan Co., Grass valley $\mathrm{CA}$ ). This orientation allows pulsed injection of ions produced in the plasma into the flight tube by pulsing the repeller plate in the ion extraction region. A pulse generated with a variable delay relative to the initiation of the RF power pulse triggers the injection. Adjustment of the repeller pulse delay permits selection of ions formed in desired time regimes during the RF power pulse cycle. Detailed information about the TOF-MS system appears in Table 4.1. More information about the instrument configuration and data acquisition also appears in previous papers. ${ }^{23-24}$

Table 4.1 TOF-MS operating parameters

\section{Time-of-Flight}

Flight path length

$1 \mathrm{~m}$

Ion lenses potential

Skimmer

$-375 \mathrm{~V}$ 
Table 4.1 TOF-MS operating parameters (continued)

\begin{tabular}{ll}
\hline Extractor & $0 \mathrm{~V}$ \\
Accelerator & $-1530 \mathrm{~V}$ \\
Deflector $(\mathrm{X} 1)$ & $-1880 \mathrm{~V}$ \\
Deflector $(\mathrm{Y} 1)$ & $-1480 \mathrm{~V}$ \\
Repeller & $+250 \mathrm{~V}$ \\
Detector & $-1895 \mathrm{~V}$ \\
Vacuum conditions & \\
Intermediate stage & $10^{-5}$ Torr \\
Flight tube & $10^{-6}$ Torr \\
\hline
\end{tabular}

A copper disk (with or without analyte loaded) is placed on the tip of a direct insertion probe (DIP) and introduced into the GD chamber through a ball valve. Cathodic sputtering is limited to the disk front surface by means of surrounding the disk with a nonconducting ceramic shield machined from MACOR (Accuratus, Washington, N.J.). Sampling distance refers to the distance from the front surface of the sample cathode to the ion exit orifice. It can be adjusted by moving the DIP position horizontally in the GD chamber and was usually within the range of $12-20 \mathrm{~mm}$ in this study. The GD powering conditions are typically 80 W RF power with a 2 ms pulse width and $10 \%$ duty cycle. Discharge gas, helium or argon (Ultra high purity, Matheson, Basking Ridge, NJ), was introduced to the GD chamber through a metering valve and the pressure was monitored by a thermocouple pressure gauge purchased from Teledyne Hastings Instruments (Hampton, VA) and Varian (Lexington, MA) respectively. Detailed GD operation conditions were provided with mass spectra shown. 


\subsubsection{Preparation of Thyroid Hormone Solutions and Residues}

3,5-Diiodo-L-thyronine (T2) (99\%), 3,3'5-Triiodo-L-thyronine(T3) (95\%), LThyroxine powders(T4) (98\%) were purchased from Sigma-Aldrich (Louis, MO). Structures are shown in Fig. 4.2. These iodinated organic compounds have extremely low water solubility. As recommended by the vendor, these compounds were dissolved in $4 \mathrm{M}$ ammonium hydroxide in methanol to make analyte solutions. The solvent was prepared by diluting $2 \mathrm{~mL}$ concentrated ammonium hydroxide (Fisher Scientific, Pittsburgh, PA) with $5.4 \mathrm{~mL}$ methanol (HPLC grade, EMD Millipore, Billerica, MA). Stock solutions of each TH compound were prepared at a concentration of $2 \mu \mathrm{g} / \mu \mathrm{L}$. Calibration standards of a set of concentrations $0.2,0.4,0.6,0.8,1.0 \mu \mathrm{g} / \mu \mathrm{L}$ were prepared from stock solutions through dilution with original solvent. For comparison purpose among the three compounds, $\mathrm{TH}$ solutions of the same concentration $(0.005 \mathrm{M})$ were prepared. Cesium chloride was spiked into the analyte solutions as internal standard at a concentration of $2 \mu \mathrm{g} / \mu \mathrm{L}$. For comparison purposes among the three compounds, $\mathrm{TH}$ solutions of the same concentration $(0.005 \mathrm{M})$ were prepared.

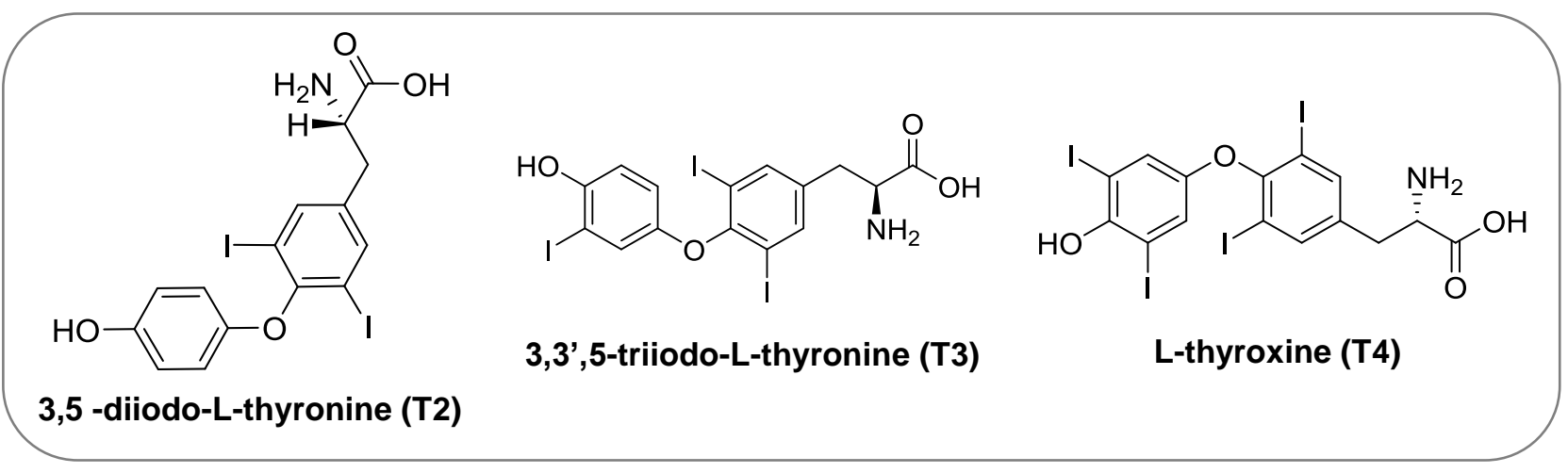

Figure 4.2 Structures of thyroid hormone compounds 
TH solutions cannot be directly analyzed by GDMS, therefore they were introduced into the ion source as dry solution residues. Samples were prepared by adding an aliquot of the analyte solution to the front surface of a supporting copper pellet which also serves as the GD cathode. The pellet, with analyte loaded, was then dried in a vacuum desiccator to remove solvent and generate a thin layer of dry residue. The dry pellet with analyte residue was introduced to the ion source for mass spectrometric analysis. It was noticed that distribution of analyte solution on the pellet surface has a significant effect on the ion signal intensity. Addition of analyte solution as a droplet on the pellet produces a relatively thick residue but occupies a small surface area. This type of residue was not favorable for the cathodic sputtering process that liberates the atoms or molecules to gaseous phase and the analyte ion signal obtained was very weak. Therefore, when adding analyte solutions, it was evenly distributed over the entire pellet surface to create a residue of a large surface area.

Residues prepared from different volumes were used for different purposes. In the entire study, analyte residues generated from either $2 \mu \mathrm{L}$ or $10 \mu \mathrm{L}$ solution deposit were used. Sample residues obtained from $10 \mu \mathrm{L}$ deposit were used for most of this study, including initial tests of analyte signal behavior during the pulse, comparison between helium and argon discharge, and comparison among the three TH compounds. Residues from $2 \mu \mathrm{l}$ deposit were only used to prepare the calibration standards and examine the quantitative ability of the system.

Analysis of TH residues in GD reveals a decreasing iodine signal over sputtering time. The normalized iodine signal drops rapidly in the first $10 \mathrm{~min}$ and then comes to a 
gentle decrease until the end of the test at about $16 \mathrm{~min}$. So, iodine signal averaged over 12-16 min was used to represent the concentration of the solution that produced the residue. lodine intensities found from different concentrations were plotted against the concentrations to build a calibration curve. For each concentration, three replicates were used.

\subsection{Results and Discussion}

\subsubsection{Helium Discharge}

Argon is commonly employed as the discharge gas in most GD studies. Because of its relatively massive nature, cathodic sputtering in an argon plasma affords high sputtering efficiency and is widely used in surface and depth profiling analysis. The discharge conditions for a stable argon plasma have been established and optimized within this self-assembled GDMS. However, because of the relatively high ionization energy of iodine (it has been noted that argon ICP does not provide sufficient ionization of iodine and it was reported that only $29 \%$ of the iodine atoms could be ionized by the argon plasma. ${ }^{25}$ In this GDMS study, helium with metastable energies around $20 \mathrm{eV}$ was proposed to create a highly energetic environment to maximize ionization. 


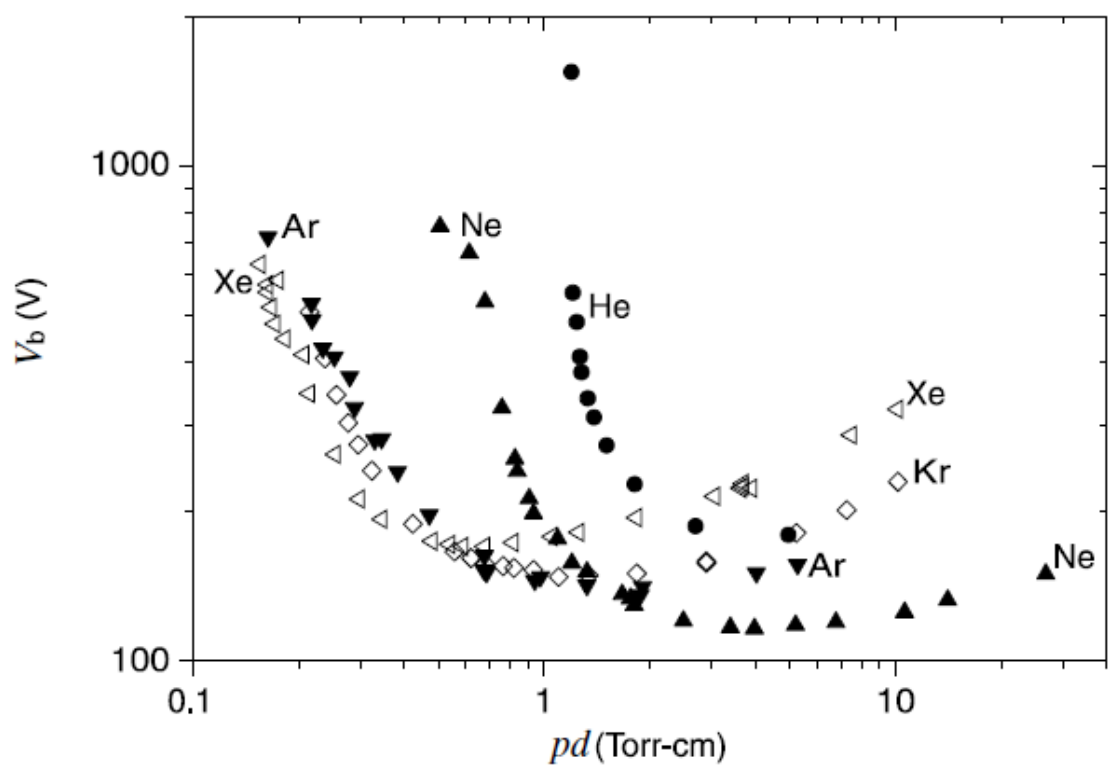

Figure 4.3 Breakdown voltage for plane-parallel electrodes for noble gases ${ }^{26}$

The use of helium gas in our GD device revealed some challenges: first, whether the instrument can achieve the requirements for a stable helium discharge, such as power, interelectrode distance and pressure; second, whether the lighter helium atoms provide sufficient sputtering of the cathode. Experiments employing a pure copper cathode were undertaken to provide operating parameters that yielded a stable helium plasma within our system. Paschen's law clearly addresses the relation of the breakdown voltage of a gas to the product of pressure and interelectrode distance. According to Paschen's curves (Fig. 4.3) describing breakdown for noble gases, one can know that breakdown of argon and helium occurs at different voltages if pressure and distance are kept identical. Based on the discharge conditions for an argon plasma, a helium plasma can be started by increasing either voltage or pressure. However, the 
power supply of the GD does not allow control of voltage directly and only permits adjustment of power in a small range (up to $140 \mathrm{~W}$ ). Therefore, increasing voltage solely relative to that of an argon discharge is not an option to initiate a helium discharge. For that reason, plasma stability was achieved by increasing the operating pressure. It was found that a helium discharge could be initiated under the similar power and distance conditions used for argon, but at significantly higher pressure minimum of 2 Torr is required in contrast to 0.3 Torr necessary for an argon discharge. Although high pressure is required to maintain helium discharge in the ion source, it did not negatively impact the high vacuum essential for ion transport and detection. The current GDMS system accommodated a helium plasma and maintained proper function.

Mass spectra were acquired to examine the sputtering and ionization properties of the helium plasma. Afterpeak spectra were collected based on the knowledge that argon plasma produces the most abundant analytical signals in the afterglow and the helium plasma was expected to behave similarly. Fig. $\mathbf{4 . 4}$ shows the mass spectrum of $\mathrm{Cu}$ collected at $2.1 \mathrm{~ms}$. Since helium is used instead of argon, argon species $\left(\mathrm{Ar}^{+}, \mathrm{Ar}_{2}{ }^{+}\right)$, potential interferences for analyte determination, disappear from the ion profile. Ion signals related to the helium gas, such as $\mathrm{He}^{+}, \mathrm{HeH}^{+}, \mathrm{He}_{2}^{+}$can be found at low masses $(\mathrm{m} / \mathrm{z} 4,5,8)$. Due to higher energy transferred from the metastable helium atoms, enhanced background species signals, $\mathrm{N}^{+}, \mathrm{O}^{+}, \mathrm{OH}^{+}, \mathrm{N}_{2}{ }^{+}, \mathrm{O}_{2}+, \mathrm{CO}_{2}+$, can be observed in the range of $m / z 14-44$. One tiny peak arises at $m / z 85$ and has not been identified yet. Other than these background signals from the discharge gas and impurities, one can see the intense $\mathrm{Cu}^{+}$ion signal at $m / z 63 \& 65$, arising from sputtering dissociation of the 
cathode material. The presence of cathode species proves the sputtering capability of a helium plasma and indicates the possible use of helium plasma in surface analysis.

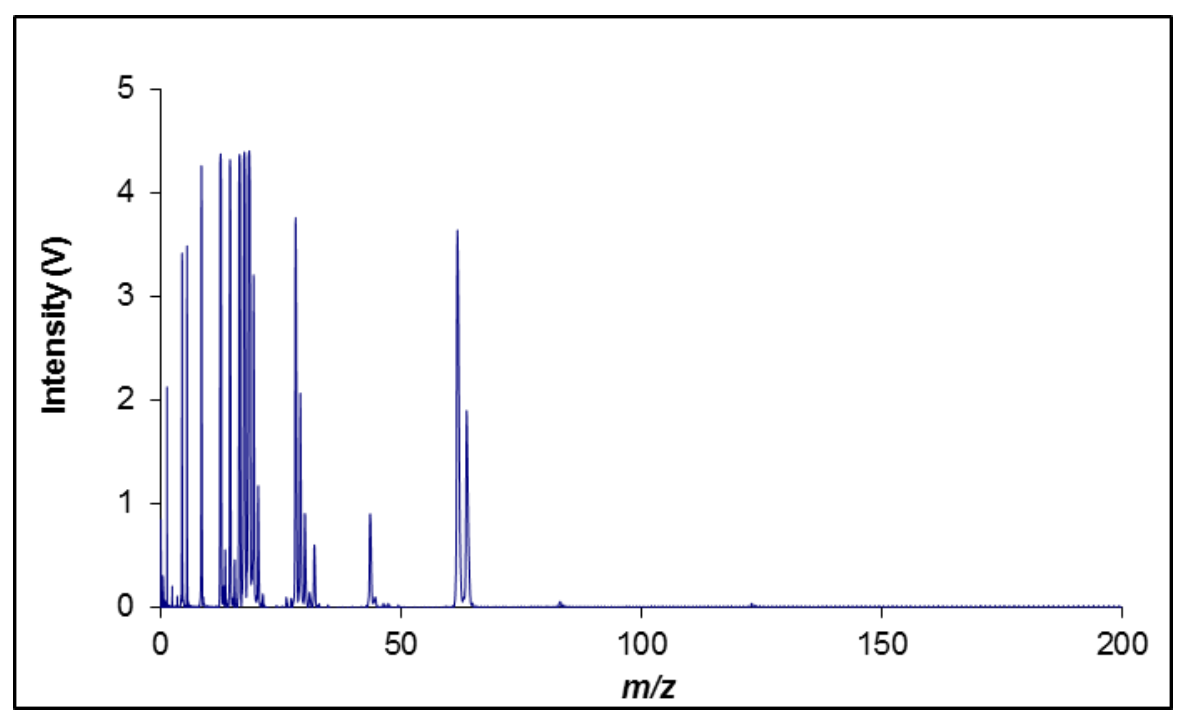

Figure 4.4 Afterpeak spectrum (2.1 ms) of $\mathrm{Cu}$ in He plasma GD operating conditions: 2.5 Torr He discharge pressure, 80 W RF power, 2 ms pulse width, $10 \%$ duty cycle and $12 \mathrm{~mm}$ sampling distance.

\subsubsection{Performance: He vs Ar}

The following work was undertaken to study the helium plasma performance in the analysis of iodinated organic compounds compared with that of an argon plasma. Extensive studies to compare helium and argon plasma properties were reported ${ }^{26}$ and this study focuses on helium plasma performance in terms of overall ion yields for these specific compounds. Residues of T3 were introduced to He and Ar plasma separately. Afterpeak spectra collected at $2.1 \mathrm{~ms}$ were displayed in Fig. 4.5. Both spectra show 
intense background species at low masses (below $m / z 50$ ). Because any potential analyte peaks in this range are subject to strong interferences from the background and can't be identified, attention was focused on the higher mass ion signals. The $\mathrm{Cu}^{+}$ion signal shows extremely high intensity in both spectra. An intense peak at $m / z 127$ is found in both helium and argon plasmas. Based on its mass-to-charge value, it is attributed to the ion signal of elemental iodine, arising from dissociation of iodine from T3 molecule. Beside these, argon plasma has an $\mathrm{Ar}_{2}{ }^{+}$at $m / z$ 80, whereas helium reveals several peaks at $m / z 67,69,83,100$. In the following analysis, these peaks show little intensity and can't be identified. Based on these findings, a comparison of the iodine intensity was carried out between the two plasmas. It is obvious that helium plasma is comparable to, or even better than argon plasma in terms of producing iodine ions. This is consistent with the finding in literature ${ }^{27}$ that a helium plasma yields the highest intensities in the analysis of Standard Reference Material (SRM) Copper 500 of all gases investigated. The observation here also addresses that the high ionizing efficiency of helium plasma could compensate any possible signal loss caused by lower sputtering efficiency. Therefore, helium was used as the discharge gas in the following work. 
(a) $\mathrm{He}$

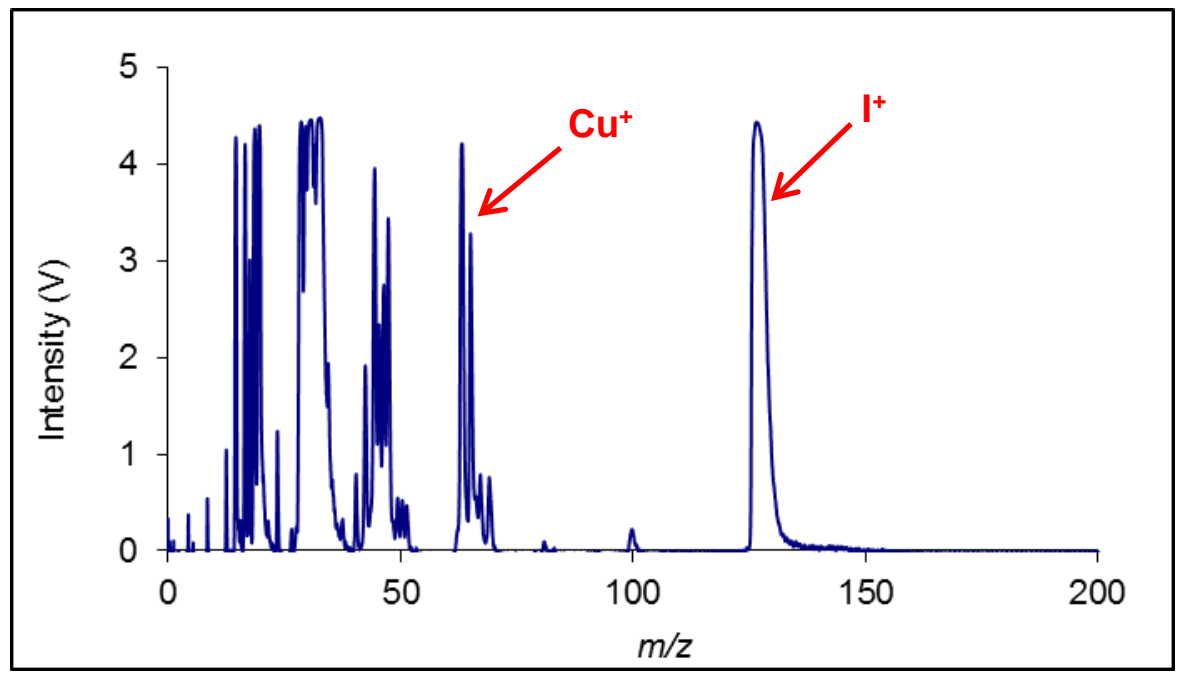

(b) $\mathrm{Ar}$

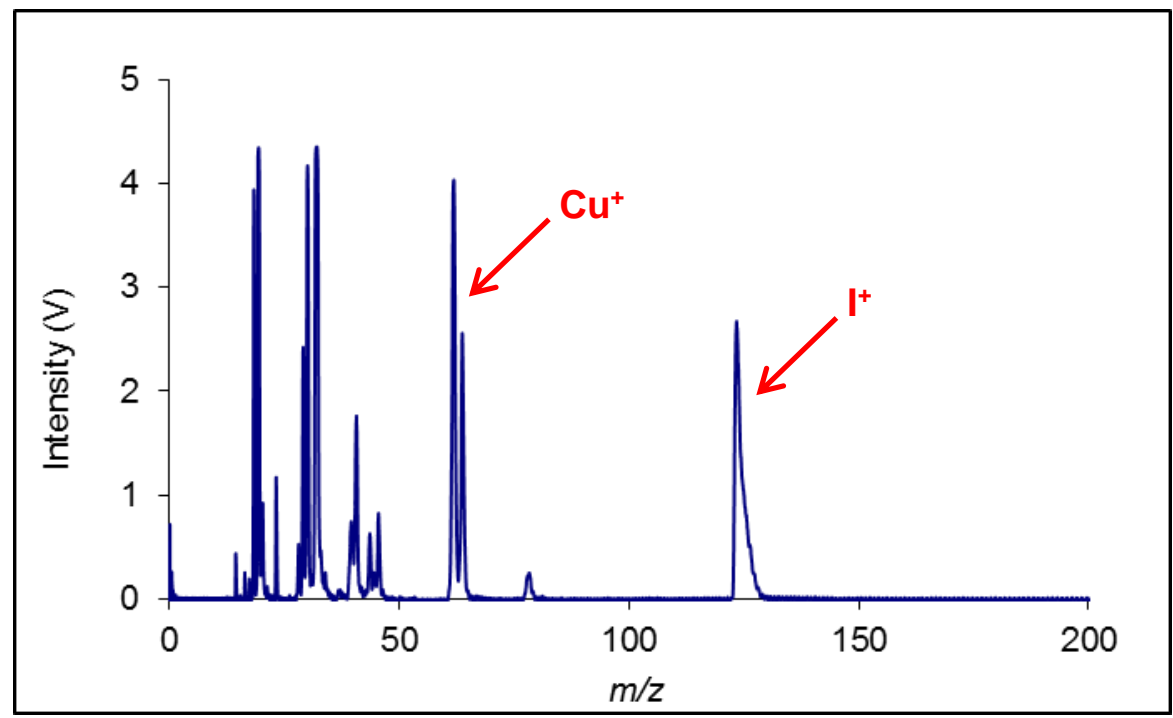

Figure 4.5 Afterpeak spectra $(2.1 \mathrm{~ms})$ of T3 residue in (a) 3 Torr He vs (b) $0.6 \mathrm{Torr} \mathrm{Ar}$ Other GD operating parameters: 100 W RF power, 2 ms pulse width, 10\% duty cycle; $17 \mathrm{~mm}$ sampling distance. 


\subsubsection{Diiodothyronine (T2) Ion Profiles Over a Pulse Cycle}

Pulsed RF power sustains the GD and this pulsed operation leads to the formation of three temporal regimes in the plasma termed prepeak, plateau and afterpeak. Ion profiles collected in each regime differ from each other due to the varying components contained in the plasma over the pulse cycle. Although afterpeak regimes produce the most abundant analytical information, prepeak and plateau spectra were collected as well to see if any other analytically useful signal arises in other portions of the pulse.

For all the compounds analyzed, a similar pattern is found along the pulse cycle. In the following discussion, T2 will be used as an example to describe the analyte behavior in each stage of a pulsed helium plasma. The prepeak spectrum of T2 is shown in Fig. 4.6 a. It was collected at $0.7 \mathrm{~ms}$ into the pulse. No signal was observed before $0.7 \mathrm{~ms}$ and this is very different from argon plasma in that signal can be seen at $0.1 \mathrm{~ms}$. In the prepeak, ion signals of the background species can be obtained, similar to those found in the afterpeak. No signal regarding the analyte can be found in prepeak. The plateau spectrum of T2 was collected at $1.9 \mathrm{~ms}$ into the pulse. As shown in Fig. $4.6 \mathrm{~b}, \mathrm{Cu}^{+}$ion signal from the cathode material arises in a naturally occurring isotopic ratio, but shows a very low abundance. A small ion signal was observed at $\mathrm{m} / \mathrm{z}$ 127 and this peak was intensively enhanced in the afterpeak spectrum (Fig. 4.6 c) collected at $2.3 \mathrm{~ms}$. It has been attributed to the ion signal of iodine atoms dissociated from the iodinated compound. The peak found at $\mathrm{m} / \mathrm{z} 133$ was the ion signal of $\mathrm{Cs}^{+}$, which functions as an internal reference to which the iodine signal can be normalized. 
Overall, T2 as well as the other two iodinated compounds are believed to undergo decomposition to a large extent in the plasma environment and only an iodine signal can be found with adequate intensity, maximizing in the afterglow.

(a) Prepeak

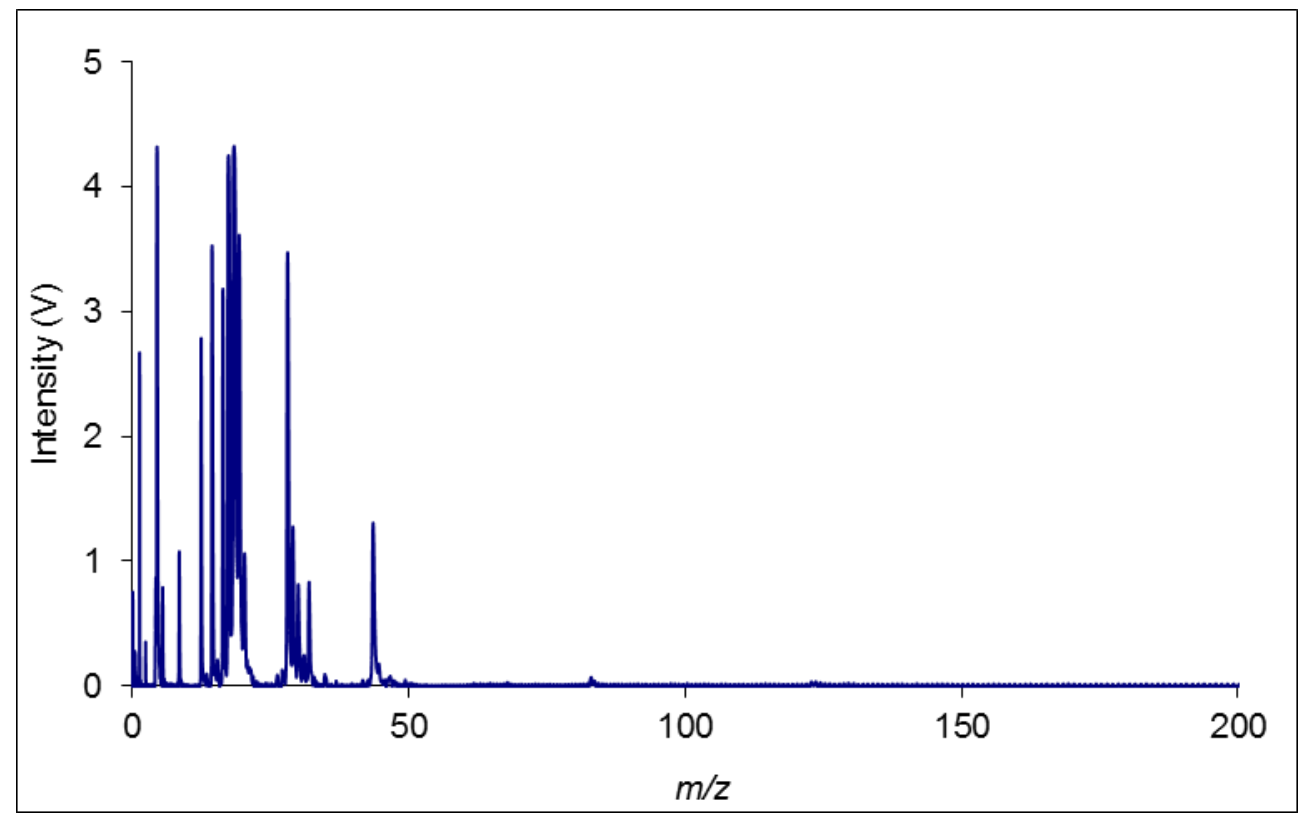

(b) Plateau

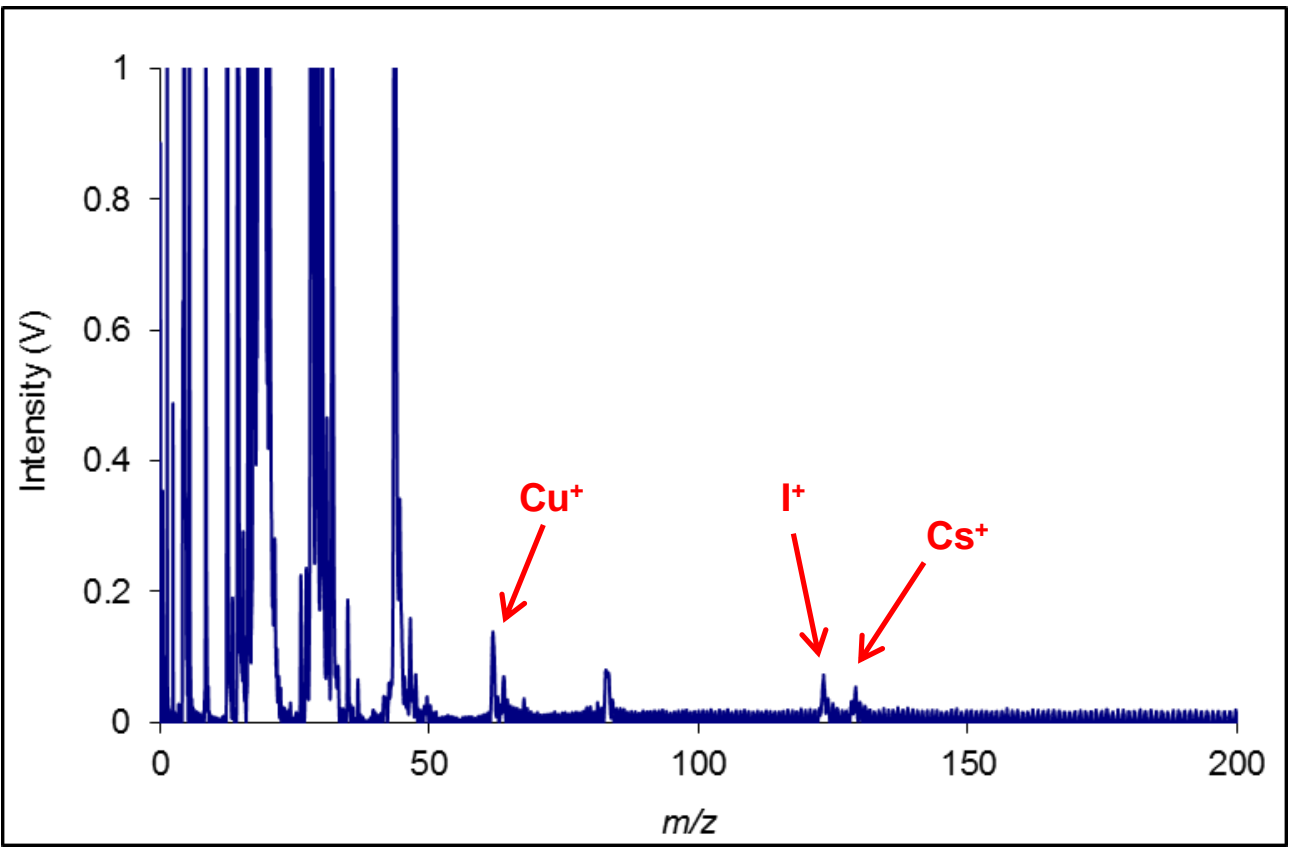


(c) Afterpeak

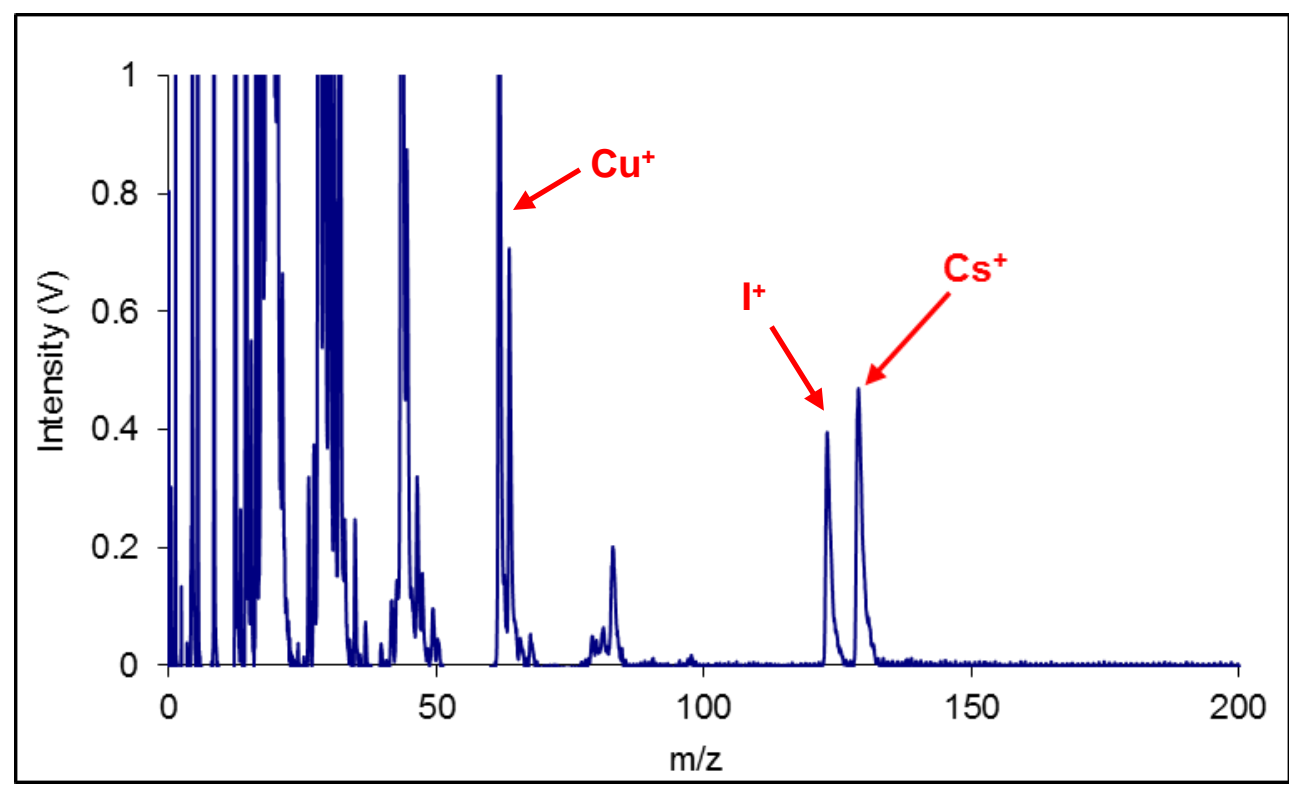

Figure 4.6 Analysis of T2 over the pulse cycle: (a) Prepeak $0.7 \mathrm{~ms}$ (b) Plateau $1.9 \mathrm{~ms}$ (c) Afterpeak $2.1 \mathrm{~ms}$ GD operating conditions: $60 \mathrm{~W}$ RF power, 2 ms pulse width, $10 \%$ duty cycle, $13 \mathrm{~mm}$ sampling distance. 2.5 Torr He discharge gas.

\subsubsection{Quantification of Diiodothyronine (T2)}

Based on the finding that iodinated compounds produce an intense iodine signal in the afterpeak, the following work was undertaken to explore the possibility of using iodine ion to determine the quantity of the organic compound. T2 solutions at five concentrations were prepared and deposited to copper disks to prepare dry residues. These residues were then analyzed by GDMS. lodine signal found for each concentration was plotted against the concentration to build a calibration curve. As shown in Fig. 4.7, the calibration curve displays good linearity between the iodine 
intensity and analyte concentration, with a correlation coefficient of 0.9918 . It validates the utility of iodine ion in quantification and demonstrates the potential of GDMS system in the quantitative determination of iodinated organic compounds.

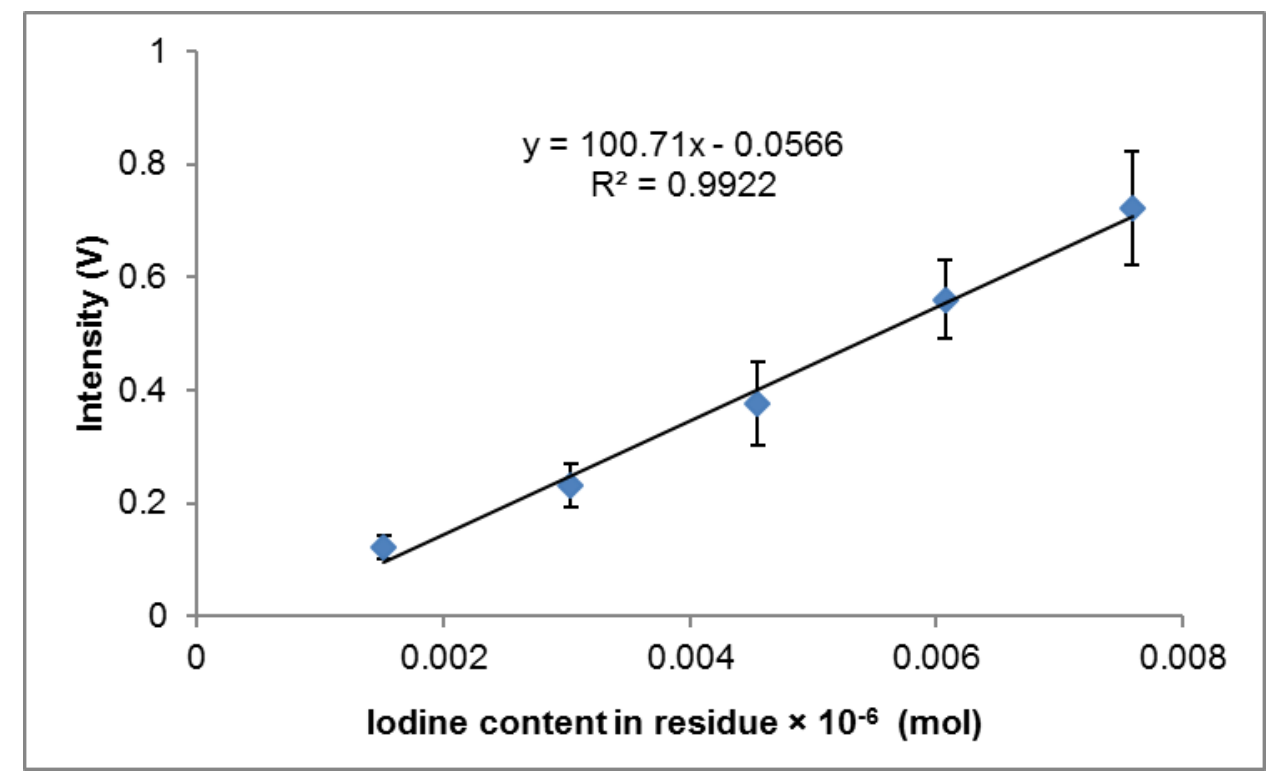

Figure 4.7 Calibration curve of diiodothyronine (T2).

\subsubsection{Comparison among Three Thyroid Hormones}

As aforementioned, an iodine signal was found in the afterpeak regime for all three iodinated compounds. These compounds (T2, T3, T4) contain 2, 3 or 4 iodine atoms on multiple positions in the molecule. The following work was carried out to investigate the relationship between the quantity of iodine atoms and its ionized signal among the three iodinated compounds. Residues containing the same number of moles for the three compounds were prepared and analyzed. The iodine signal for 
each compound is shown in Fig. 4.8. T4 produces the highest signal because it contains the highest amount (4 portions) of iodine atoms. T2 contains the lowest amount ( 2 portions) and thus yields the lowest signal. In addition, one can see from the graph that iodine intensities for the three compounds display a proportional increase relationship. This is even clear if the iodine signal is plotted against the iodine quantity (Fig. 4.9). A linear relationship is found between the iodine quantity and intensity. With blank included, $R^{2}=0.9997$; without blank, $R^{2}=0.9998$. Although only three $\mathrm{TH}$ compounds are involved in this test, the excellent linearity clearly indicates that iodine atoms existed at different positions within organic molecules are ionized to the same extent. lodine ion intensity is more dependent on the quantity of iodine atoms existed in the sample, while the position of iodine atoms has little effect on the ultimate ion signal.

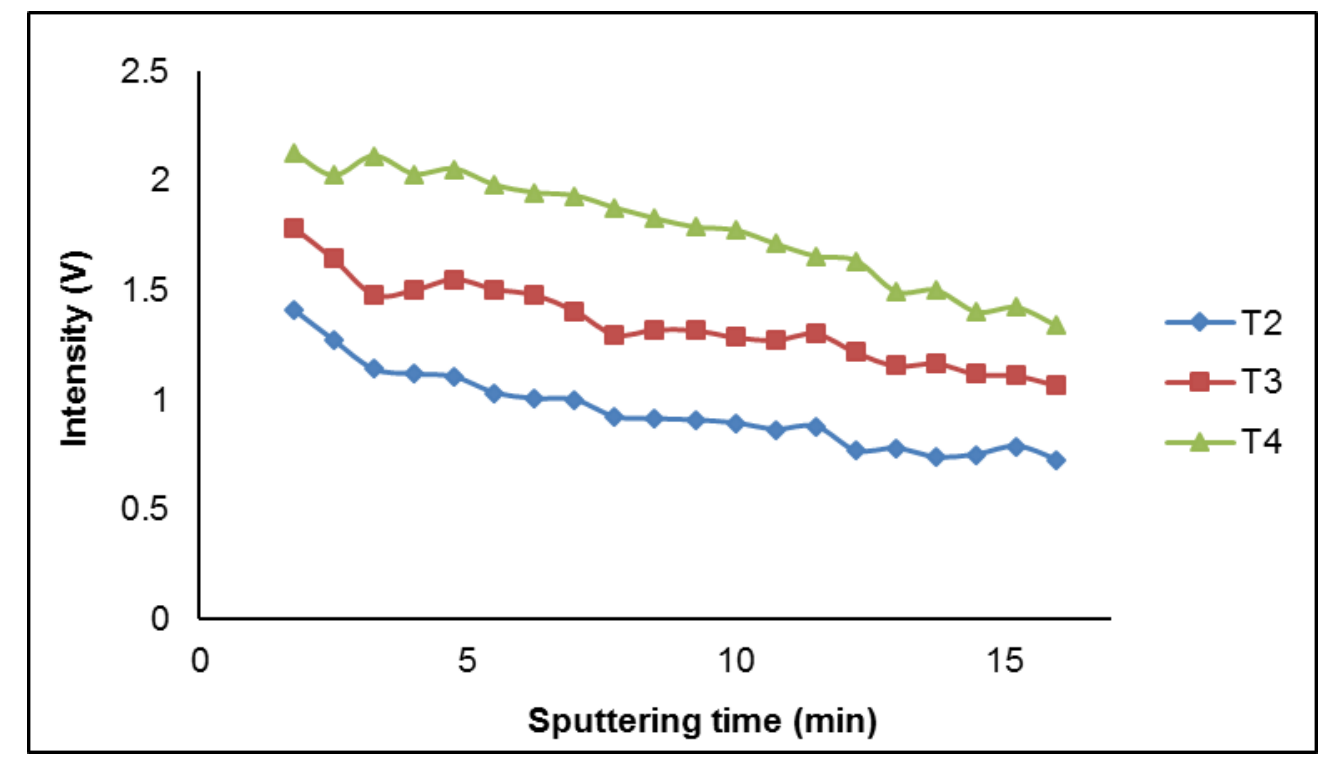

Figure 4.8 lodine ion intensity over sampling time for TH compounds; GD operating conditions: $70 \mathrm{~W}$ RF power, $2 \mathrm{~ms}$ pulse width, $10 \%$ duty cycle, $18 \mathrm{~mm}$ sampling distance, 2.2 Torr He. 


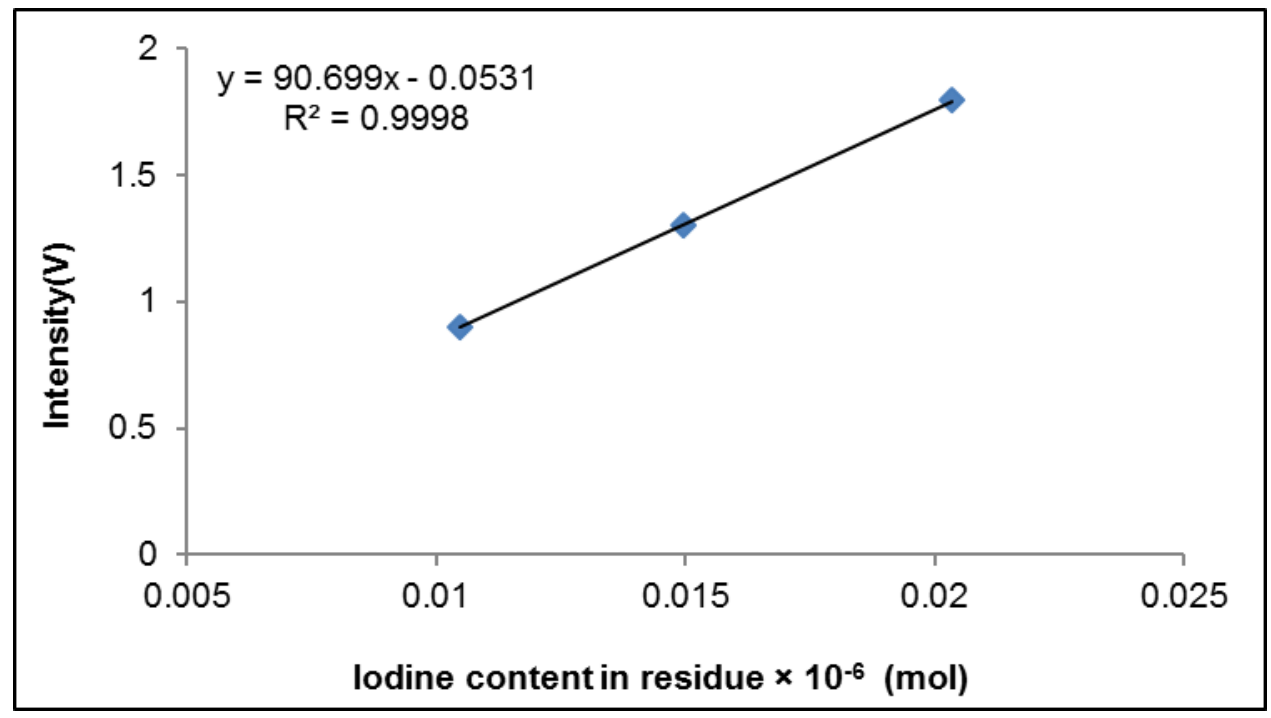

Figure 4.9 Linear relationship among three TH compounds.

With that, one can further think about the process that iodine atoms go through to become ionized. It is known that cathodic sputtering releases gaseous analyte to the negative glow for ionization. The species released could be molecular species, large fragments or individual atoms. For the $\mathrm{TH}$ compounds, if molecular species or large fragments are being liberated during sputtering, one would expect the large species go through differential collisional dissociation in the plasma and eventually release free iodine atoms to a different extent for each position. The result would be a different population of iodine atoms released, leading to a different ionizing efficiency for each position. A linear relationship between iodine quantity and intensity among the three compounds is unlikely to occur. Therefore, it seems more likely that a majority of the iodine atoms are released to the plasma directly in the sputtering step, which shows no structural preference. These free iodine atoms then undergo similar collisional 
ionization. The efficiency of sputtering and ionization is consistent for iodine attached in different positions and the resulting ion signal is then independent of its structural position within the molecule.

\subsection{Conclusion}

A helium glow discharge is achievable in the current GDMS system under the similar GD conditions as argon but at a higher pressure. Application of the helium plasma to the analysis of iodinated organic compounds, in a form of dry residues, reveals an intense iodine signal in the afterglow. Comparison of this iodine signal with that found in an argon plasma has proved the efficiency of helium plasms in sputtering and ionizing iodine atoms. Moreover, iodine intensity correlates well with the quantity of iodinated compounds in the residue and the calibration curve generated exhibits good linearity, suggesting the applicability of using iodine ion as a characteristic signal for quantification of iodinated compounds. Comparison of the iodine signals from $\mathrm{TH}$ compounds containing different numbers of iodine atoms further confirms that iodine atoms found in different positions display similar ionization efficiency and the corresponding iodine intensity is solely related to the iodine quantity in the source, regardless of its position within the molecule. This study demonstrates the potential of GDMS in the quantitative analysis of iodinated organic compounds through the measurement of elemental iodine. 


\subsection{References}

1. Warner, A.; Mittag, J., Thyroid hormone and the central control of homeostasis. Journal of molecular endocrinology 2012, 49 (1), R29-35.

2. Hetzel, B. S., lodine deficiency disorders (IDD) and their eradication. Lancet (London, England) 1983, 2 (8359), 1126-9.

3. Chow, C. C.; Phillips, D. I.; Lazarus, J. H.; Parkes, A. B., Effect of low dose iodide supplementation on thyroid function in potentially susceptible subjects: are dietary iodide levels in Britain acceptable? Clinical endocrinology 1991, 34 (5), 413-6.

4. Edmonds, J. S.; Morita, M., The determination of iodine species in environmental and biological samples (Technical Report). In Pure Appl. Chem., 1998; Vol. 70, p 1567.

5. Moreda-Pineiro, A.; Romaris-Hortas, V.; Bermejo-Barrera, P., A review on iodine speciation for environmental, biological and nutrition fields. J. Anal. At. Spectrom. 2011, $26(11), 2107-2152$.

6. Knapp, G.; Maichin, B.; Fecher, P.; Hasse, S.; Schramel, P., lodine determination in biological materials Options for sample preparation and final determination. Fresenius' Journal of Analytical Chemistry 1998, 362 (6), 508-513.

7. Bermejo-Barrera, P.; Aboal-Somoza, M.; Bermejo-Barrera, A., Atomic absorption spectrometry as an alternate technique for iodine determination (1968-1998). J. Anal. At. Spectrom. 1999, 14 (7), 1009-1018.

8. Anderson, K. A.; Markowski, P., Speciation of iodide, iodine, and iodate in environmental matrixes by inductively coupled plasma atomic emission spectrometry using in situ chemical manipulation. J. AOAC Int. 2000, 83 (1), 225-30. 
9. Varga, I., lodine determination in dietary supplement products by TXRF and ICPAES spectrometry. Microchem. J. 2007, 85 (1), 127-131.

10. Oliveira, A. A.; Trevizan, L. C.; Nóbrega, J. A., REVIEW: lodine Determination by Inductively Coupled Plasma Spectrometry. Applied Spectroscopy Reviews 2010, 45 (6), 447-473.

11. Franke, K.; Meyer, U.; Wagner, H.; Hoppen, H. O.; Flachowsky, G., Effect of various iodine supplementations, rapeseed meal application and two different iodine species on the iodine status and iodine excretion of dairy cows. Livestock Science 2009, $125(2-3), 223-231$.

12. Schone, F.; Leiterer, M.; Lebzien, P.; Bemmann, D.; Spolders, M.; Flachowsky, G., lodine concentration of milk in a dose-response study with dairy cows and implications for consumer iodine intake. J. Trace Elem. Med Biol. 2009, 23 (2), 84-92.

13. Leiterer, M.; Truckenbrodt, D.; Franke, K., Determination of iodine species in milk using ion chromatographic separation and ICP-MS detection. European Food Research and Technology 2001, 213 (2), 150-153.

14. Sun, M.; Gao, Y.; Wei, B.; Wu, X., Determination of iodine and bromine in coal and atmospheric particles by inductively coupled plasma mass spectrometry. Talanta 2010, $81(1-2), 473-476$.

15. Grinberg, P.; Sturgeon, R. E., Ultra-trace determination of iodine in sediments and biological material using UV photochemical generation-inductively coupled plasma mass spectrometry. Spectrochimica Acta Part B: Atomic Spectroscopy 2009, 64 (3), 235-241. 
16. Romarís-Hortas, V.; Moreda-Piñeiro, A.; Bermejo-Barrera, P., Microwave assisted extraction of iodine and bromine from edible seaweed for inductively coupled plasma-mass spectrometry determination. Talanta 2009, 79 (3), 947-952.

17. Michalke, B.; Schramel, P.; Witte, H., lodine speciation in human serum by reversed-phase liquid chromatography-ICP-mass spectrometry. Biol. Trace Elem. Res. 2000, $78(1-3), 81-91$.

18. Michalke, B.; Schramel, P., lodine speciation in biological samples by capillary electrophoresis-inductively coupled plasma mass spectrometry. Electrophoresis 1999, 20 (12), 2547-53.

19. Gilfedder, B. S.; Petri, M.; Wessels, M.; Biester, H., An iodine mass-balance for Lake Constance, Germany: Insights into iodine speciation changes and fluxes. Geochim. Cosmochim. Acta 2010, 74 (11), 3090-3111.

20. Kaňa, A.; Hrubá, L.; Vosmanská, M.; Mestek, O., Analysis of iodine and its species in animal tissues. Chemical Speciation \& Bioavailability 2015, 27 (2), 81-91.

21. Simon, R.; Tietge, J.; Michalke, B.; Degitz, S.; Schramm, K. W., lodine species and the endocrine system: thyroid hormone levels in adult Danio rerio and developing Xenopus laevis. Analytical and bioanalytical chemistry 2002, 372 (3), 481-485.

22. Wilson, R. A.; Yanes, E. G.; Kemppainen, R. J., lodine speciation in dog foods and treats by high performance liquid chromatography with inductively coupled plasma mass spectrometry detection. Journal of chromatography. B, Analytical technologies in the biomedical and life sciences 2016, 1022, 183-90. 
23. Zhang, N.; King, F. L., Direct manganese (Mn) speciation in solid state materials by pulsed glow discharge time-of-flight mass spectrometry. J. Anal. At. Spectrom. 2009, $24(11), 1489-1497$.

24. Gu, G.; DeJesus, M.; King, F. L., Direct FexOy speciation in solid state materials by pulsed millisecond radio frequency glow discharge time-of-flight mass spectrometry. J. Anal. At. Spectrom. 2011, 26 (4), 816-821.

25. Montaser, A., Inductively coupled plasma mass spectrometry. John Wiley \& Sons: 1998.

26. Lieberman, M. A.; Lichtenberg, A. J., Principles of plasma discharges and materials processing. John Wiley \& Sons: 2005.

27. Mohill, M. L., Sputtering and ionization by helium and argon in the microsecond pulsed glow discharge using time-of-flight mass spectrometry. 2001.

28. Giglio, J. J.; Caruso, J. A., Evaluation of Alternative Plasma Gases for RadioFrequency Glow Discharge Mass Spectrometry (rf GD-MS). Appl. Spectrosc. 1995, 49 (7), 900-906. 
Glow Discharge Mass Spectrometric Determination of Selected Biological Species

\section{Xiaqing $\mathbf{X u}$}

Dissertation submitted to the Eberly College of Arts and Sciences at West Virginia University in partial fulfillment of the requirements for the degree of

Doctor of Philosophy in Chemistry

C. Eugene Bennett Department of Chemistry

APPROVAL OF THE EXAMINING COMMITTEE
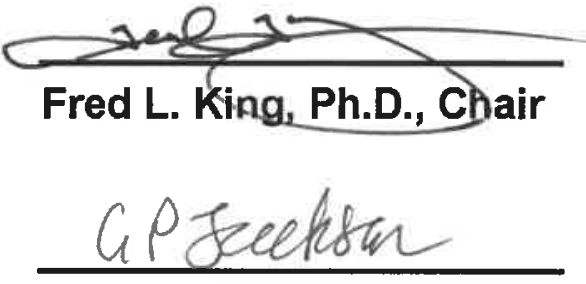

Glen Jackson, Ph.D.
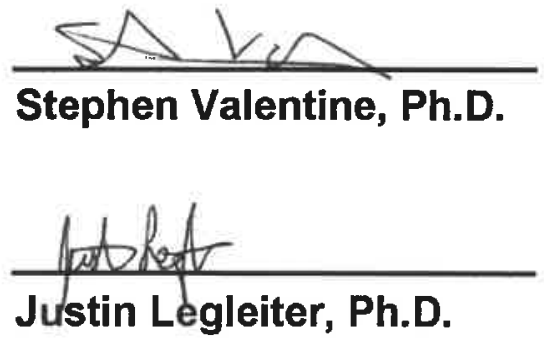

$\frac{7 / 18 / 2017}{\text { Date }}$

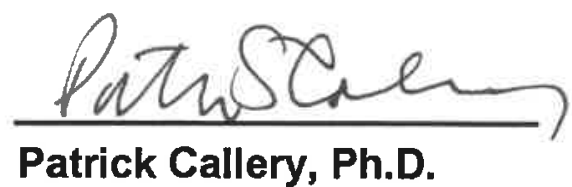

